COMPUTER SCIEIVUL'

\title{
COMPUTER PERIPHERAL MEMORY SYSTEM FORECAST
}

$-Q C$ 100

.457

\#500-45

1979

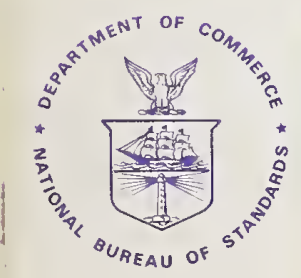

NBS Special Publication 500-45

U.S. DEPARTMENT OF COMMERCE National Bureau of Standards 


\section{NATIONAL BUREAU OF STANDARDS}

The National Bureau of Standards' was established by an act of Congress March 3, 1901. The Bureau's overall goal is to strengthen and advance the Nation's science and technology and facilitate their effective application for public benefit. To this end, the Bureau conducts research and provides: (1) a basis for the Nation's physical measurement system, (2) scientific and technological services for industry and government, (3) a technical basis for equity in trade, and (4) technical services to promote public safety. The Bureau's technical work is performed by the National Measurement Laboratory, the National Engineering Laboratory, and the Institute for Computer Sciences and Technology.

THE NATIONAL MEASUREMENT LABORATORY provides the national system of physical and chemical and materials measurement; coordinates the system with measurement systems of other nations and furnishes essential services leading to accurate and uniform physical and chemical measurement throughout the Nation's scientific community, industry, and commerce; conducts materials research leading to improved methods of measurement, standards, and data on the properties of materials needed by industry, commerce, educational institutions, and Government; provides advisory and research services to other Government Agencies; develops, produces, and distributes Standard Reference Materials; and provides. calibration services. The Laboratory consists of the following centers:

Absolute Physical Quantities ${ }^{2}$ - Radiation Research - Thermodynamics and Molecular Science - Analytical Chemistry - Materials Science.

THE NATIONAL ENGINEERING LABORATORY provides technology and technical services to users in the public and private sectors to address national needs and to solve national problems in the public interest; conducts research in engineering and applied science in support of objectives in these efforts; builds and maintains competence in the necessary disciplines required to carry out this research and technical service; develops engineering data and measurement capabilities; provides engineering measurement traceability services; develops test methods and proposes engineering standards and code changes; develops and proposes new engineering practices; and develops and improves mechanisms to transfer results of its research to the utlimate user. The Laboratory consists of the following centers:

Applied Mathematics - Electronics and Electrical Engineering 2 - Mechanical Engineering and Process Technology ${ }^{2}-$ Building Technology - Fire Research Consumer Product Technology — Field Methods.

THE INSTITUTE FOR COMPUTER SCIENCES AND TECHNOLOGY conducts research and provides scientific and technical services to aid Federal Agencies in the selection, acquisition, application, and use of computer technology to improve effectiveness and economy in Government operations in accordance with Public Law 89-306 (40 U.S.C. 759), relevant Executive Orders, and other directives; carries out this mission by managing the Federal Information Processing Standards Program, developing Federal ADP standards guidelines, and managing Federal participation in ADP voluntary standardization activities; provides scientific and technological advisory services and assistance to Federal Agencies; and provides the technical foundation for computer-related policies of the Federal Government. The Institute consists of the following divisions:

Systems and Software - Computer Systems Engineering - Information Technology.

${ }^{1}$ Headquarters and Laboratories at Gaithersburg, Maryland, unless otherwise noted; mailing address Washington,D.C. 20234.

${ }^{2}$ Some divisions within the center are located at Boulder, Colorado, 80303. 


\section{COMPUTER SCIENCE \& TECHNOLOGY:}

\section{Computer Peripheral Memory System Forecast}

Robert B. J. Warnar, Peter J. Calomeris, and Steve A. Recicar

Institute for Computer Sciences and Technology

National Bureau of Standards

Washington, DC 20234

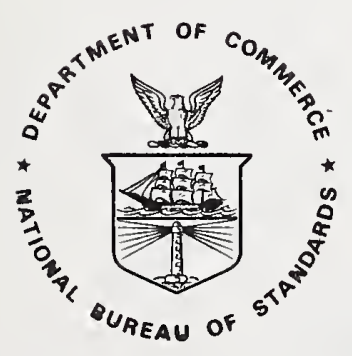

U.S. DEPARTMENT OF COMMERCE, Juanita M. Kreps, Secretary Jordan J. Baruch, Assistant Secretary for Science and Technology NATIONAL BUREAU OF STANDARDS, Ernest Ambler, Director Issued April 1979 


\section{Reports on Computer Science and Technology}

The National Bureau of Standards has a special responsibility within the Federal Government for computer science and technology activities. The programs of the NBS Institute for Computer Sciences and Technology are designed to provide ADP standards, guidelines, and technical advisory services to improve the effectiveness of computer utilization in the Federal sector, and to perform appropriate research and development efforts as foundation for such activities and programs. This publication series will report these NBS efforts to the Federal computer community as well as to interested specialists in the academic and private sectors. Those wishing to receive notices of publications in this series should complete and return the form at the end of this publication.

\section{National Bureau of Standards Special Publication 500-45}

Nat. Bur. Stand. (U.S.), Spec. Publ. 500-45, 147 pages (Apr. 1979) CODEN: XNBSAV

\section{Library of Congress Catalog Card Number: 79-600036}

\section{U.S. GOVERNMENT PRINTING OFFICE}

WASHINGTON: 1979

For sale by the Superintendent of Documents, U.S. Government Printing Office, Washington, D.C. 20402 Stock No. 003-003-02039-7 Price \$3.25

(Add 25 percent additional for other than U.S. mailing) 


\section{NOTE}

Reports on commercial developments included in this publication were obtained from the open literature, supplemented in most cases by site visits and meetings with appropriate company representatives. No proprietary information of any kind is included. Opinions about comparative merits of the developments were found in the published literature, and were not generated by the authors of this report. Inclusions or exclusions of specific developments are strictly reflections of the published literature. NBS did not experimentally verify the accuracy of the statements from the published literature or any of the companies cited. Specific references to companies and developments in this report cannot be construed as recommendation or endorsement by the National Bureau of Standards. 


\section{PREFACE}

The Institute for Computer Sciences and Technology (ICST), National Bureau of Standards (NBS), develops and recommends Federal Information Processing Standards; provides advisory and consultative services to other agencies of Government to support the formulation of ADP management and procurement policies and to assist in solving specific computer application problems; and conducts research and provides technical services designed to aid Government agencies in improving cost effectiveness in the selection, acquisition, and utilization of automatic data processing equipment. In the course of discharging these responsibilities, ICST staff members prepare technical reports, technology assessments, and summaries or proceedings of conferences and symposia which the Institute sponsors and conducts. Resulting publications may appear in NBS publications media, in professional or technical journals, or as separate volumes such as proceedings or monographs.

This forecast of computer peripheral memory systems was prepared as a preliminary input for the identification of areas for possible future standards development. Conclusions drawn from current trends in these technologies, such as are reflected in this report, will be a basis for standards development.

Also, this forecast was prepared as a service to Federal computer managers who are active in the ADP management field. In order to improve the utility of this information, this publication has incorporated a section with decision trees which are intended to perform as aids in the purchasing phase of computer peripheral memory equipment. The information offered by the decision trees, technical parameter tables, market trend graphs, and associated text is made available to keep the Federal ADP manager current with changes in contemporary developments and the emerging of equipments in computer peripheral memory systems.

The Institute for Computer Sciences and Technology, National Bureau of Standards, acknowledges its appreciation to all the participants in this technological forecast. Many individuals and companies have contributed information, ideas, constructive criticisms and their talents, all of which were indispensible for the completion of this document.

The authors would like to mention the invaluable contribution made by the numerous manufacturers in the generation, assembly and review of the Computer Peripheral Memory Forecast. We would like to provide special credit to Barbara Moran and Walter Lee for compiling the data for our document, to Dennis Donovan for preparing our figures, to Jaqueline Jones for assistance in the off-line data search, and to Barbara Peterson, Joan Knoerdel, and Terri L. Rau for inputting and editing the report using an on-line text editing system. 
Note.................... iii

Preface .................. . . . . iv

Table of Contents . . . . . . . . . . . . . v v

List of Tables.................... . . ix

List of Figures . . . . . . . . . . . . . . $x$

Abstract . . . . . . . . . . . . . . . . 1

I. Introduction to Computer Peripheral Memory Systems . . . . . . I I

A. Categories ................. 1

1. Rotating cylinder or disk memory systems ...... . 1

a. Drums . . . . . . . . . . . . . . 2

b. Disks................. . . 2

(1) Moving-head disks .......... 3

(2) Stationary-head disks ......... 3

2. Tape storage memory systems ........... . 4

a. Mass tapes . . . . . . . . . . . . . . 4

(1) 1.27 Centimeters $(1 / 2$ Inch) ....... 4

(2) other .............. 4

b. Cartridges ............. . . 5

c. Cassettes .............. . . . 6

d. Floppy disks .............. . . 6

3. Optical data storage memory systems--Laser beam . . . 7

4. Solid-state peripheral memory systems . . . . . . 7

a. Charge-coupled devices . . . . . . . . . . 7

b. Magnetic bubble memories .......... . 7

c. MOS (metal-oxide-semiconductor) memories ..... 8

5. Random-access magnetic memory systems--

Core memories ................. 8

6. Alternate memory systems ........... . . 9

a. Videodisk memory systems . . . . . . . . . . 9

b. Beam addressable memory systems......... 9

II. Development--Parameters of the Various Peripheral Memory

Systems 
1. Drums and Disks................ 11

a. Drums . . . . . . . . . . . . 12

b. Moving-head disks (fixed media)........ 12

c. Moving-head disks (removeable media) . . . . . 15

d. Stationary-head disks (fixed media) . . . . . . 15

e. Cartridge-disks . . . . . . . . . 15

2. Mass storage systems ............. . 19

3. Cartridges and Minicartridges ........ . 20

4. Cassettes and Minicassettes ........... 20

5. Floppy disks and minifloppy disks......... 23

6. Laser beam ............... 27

7. Charge-coupled devices ........... 29

8. Magnetic bubbles ............. 32

9. MOS .......................... 34

10. Cores.................. . . 37

11. Videodisks .. . . . . . . . . . . . 39

12. Beam addressables .............. . . 41

III. Conclusions for Each Peripheral Memory System . . . . . . 44

A. Advantages and disadvantages of the described peripheral memories--A comparison of price/performance characteristics of peripheral memories . . . . . . . . . . 44

1. Rotating cylinder or disk memory systems . . . . . 44

a. Drums . . . . . . . . . . . . . 44

b. Disks . . . . . . . . . . . . . . 44

(1) Moving-head disks .......... . . . 44

(2) Stationary-head disks ........ 45

2. Tape storage memory systems ........... 45

a. Mass tapes .. . . . . . . . . . . 45

b. Cassettes .............. . . 4 47

c. Cartridges .. . . . . . . . . . . . 48

d. Floppy disks .............. 48

3. Optical data storage memory systems--Laser beam ... 49 
4. Solid-state peripheral memory systems . . . . . . 49

a. Charge-coupled devices . . . . . . . . . 49

b. Magnetic bubble memories . . . . . . . . 50

c. MOS (metal-oxide-semiconductor) memories .... 50

5. Random-access magnetic memory systems--Core memories . . . . . . . . . . . . . 51

6. Alternate memory systems . . . . . . . . . 51

a. Videodisk memory systems . . . . . . . . . 51

b. Beam addressable memory systems ........ 52

B. Applications of the various peripheral memory systems-major and minor applications . . . . . . . . . 52

1. Rotating cylinder or disk memory systems . . . . . 52

a. Drums . . . . . . . . . . . . . . 52

b. Disks . . . . . . . . . . . . . 53

(1) Moving-head disks .......... . . 53

(2) Stationary-head disks ........ . 53

2. Tape storage memory systems . . . . . . . . 53

a. Mass tapes . . . . . . . . . . . . 53

b. Cartridges/minicartridges . . . . . . . 54

c. Cassettes/minicassettes . . . . . . . . . . 55

d. Floppy disks/minifloppy disks .......... 56

3. Optical data storage memories systems--Laser beam . . 57

4. Solid-state peripheral memory systems . . . . . . 57

a. Charge-coupled devices . . . . . . . . . 57

b. Magnetic bubble memories . . . . . . . . . . 58

c. MOS (metal-oxide-semiconductor) memories . . . 59

5. Random-access magnetic memory systems--Core memories . . . . . . . . . . . . . 59

6. Alternate memory systems . . . . . . . . . . 59

a. Videodisk memory systems . . . . . . . . . . 59

b. Beam addressable memory systems ......... 60

C. Projections for various peripheral memory technologies-current trends in developments and markets ....... 61

1. Rotating cylinder or disk memory systems . . . . . 61 a. Drums . . . . . . . . . . . . . 61

b. Disks .. . . . . . . . . . . . 61 
2. Tape storage memory systems . . . . . . . . 67

a. Mass tapes . . . . . . . . . . . 67

b. Cartridges . . . . . . . . . . . 68

c. Cassettes .............. . . 72

d. Floppy disks .............. . . 74

e. Summary graph of tape technologies . . . . . 76

3. Optical data storage memory systems--Laser beam . . . 79

4. Solid-state peripheral memory systems . . . . . 80

5. Random-access magnetic memory systems--Core memories . . . . . . . . . . . . 86

6. Alternate memory systems . . . . . . . . . . 89

a. Videodisk memory systems . . . . . . . . . . 89

b. Beam addressable memory systems ......... 90

IV. Summary and Overall Conclusions . . . . . . . . . . 91

A. A summary table of the various peripheral memory technologies ............... . 91

B. Conclusions ................ . . 95

V. Bibliography ................. 117 


\section{List of Tables}

Page

Table 01 - Drums . . . . . . . . . . . . . . . . . . 13

Table 02 - Moving-Head Disks - Fixed Media . . . . . . . . . . . . 14

Table 03 - Moving-Head Disks - Removeable Media . . . . . . . . 16

Table 04 - Stationary-Head Disks - Fixed Media . . . . . . . . . . 17

Table 05 - Cartridge Disks . . . . . . . . . . . . . . . . 18

Table 06 - Tape Mass Storage Systems . . . . . . . . . . . . . 21

Table 07 - Cartridge and Minicartridge Drives . . . . . . . . . 22

Table 08 - Cassette and Minicassette Drives . . . . . . . . . . . 24

Table 09 - Floppy Disk and Minifloppy Disk Drives . . . . . . . . 25

Table 10 - Laser Mass Memory System . . . . . . . . . . . . 28

Table 11 - Charge-Coupled Devices . . . . . . . . . . . . . . 30

Table 12 - Magnetic Bubbles . . . . . . . . . . . . . . . . 33

Table 13 - Metal-Oxide-Semiconductor (MOS) . . . . . . . . . . 35

Table 14 - Cores . . . . . . . . . . . . . . . . . . 38

Table 15 - Videodisks . . . . . . . . . . . . . . . . . . 40

Table 16 - Beam Addressables . . . . . . . . . . . . . . . . . 42

Table 17 - Typical Values and Features of Computer Memories . . . 92 


\section{List of Figures}

Figure 1 - Drums Shipped by U.S. Manufacturers . . . . . . . . 62

Figure 2 - Disks Shipped by U.S. Manufacturers . . . . . . . . 66

Figure 3 - U.S. OEM 1.27 centimeters (0.5 inch) Tape Drive Market ................ . . 6 69

Figure 4 - U.S. Tape Cartridge Drive Market . . . . . . . . . 71

Figure 5 - U.S. Cassette Drive Market . . . . . . . . . . . . 73

Figure 6-U.S. Floppy Disk Drive Market . . . . . . . . . . . 75

Figure 7 - Summary Graph of U.S. Tape Cartridge, Cassette, Floppy Disk, and OEM 1.27 centimeters (0.5 inch) Tape Drive Systems . . . . . . . . . . . 77

Figure 8 - Estimated CCD Memory Market Trends . . . . . . . . 82

Figure 9 - Estimated Magnetic Bubble Memory Market Trends . . . 84

Figure 10 - Estimated MOS Memory Market Trends . . . . . . . . . 85

Figure 11 - Estimated Core Memory Market Trends . . . . . . . . . 88

Figure 12 - Upgrade a Large Computer System - Increase On-Line Memory Capacity . . . . . . . . . . . 97

Figure 13 - Upgrade a Large Computer System - Increase Off-Line Memory Capacity .. . . . . . . . . . . 99

Figure 14 - Upgrade a Large Computer System - Increase Computer Thruput Via Fast Auxiliary Memory . . . . . . . . 100

Figure 15 - Upgrade a Large Computer System - Replacement of Peripheral Memory System ............ 101

Figure 16 - Upgrade a Small Computer System - Increase On-Line Memory Capacity . . . . . . . . . . . 102

Figure 17 - Upgrade a Small Computer System - Increase Off-Line Memory Capacity . . . . . . . . . . . 104

Figure 18 - Upgrade a Small Computer System - Increase Computer Thruput Via Fast Auxiliary Memory . . . . . . . . 105

Figure 19 - Upgrade a Small Computer System - Replacement of Peripheral Memory System ............ 106

Figure 20 - Purchasing a Large Computer System - Determine On-Line Memory Capacity . . . . . . . . . . 108

Figure 21 - Purchasing a Large Computer System - Determine Off-Line Memory Capacity . . . . . . . . . . . . 110

Figure 22 - Purchasing a Large Computer System - Increase Computer Thruput Via Fast Auxiliary Memory . . . . . . 111

Figure 23 - Purchasing a Small Computer System - Determine On-Line Memory Capacity . . . . . . . . . . 112

Figure 24 - Purchasing a Small Computer System - Determine OffLine Memory Capacity .. . . . . . . . . . . . 114

Figure 25 - Purchasing a Small Computer System - Increase Computer Thruput Via Fast Auxiliary Memory . . . . . . . 116 


\author{
Robert B. J. Warnar \\ Peter J. Calomeris \\ Steve A. Recicar
}

\begin{abstract}
This document describes and forecasts computer peripheral memory technologies as displayed by U.S. research and manufacturing facilities. Specifically, both technical and economic criteria are discussed. The presented peripheral memories include contemporary and emerging systems, all of which are compared in graphs, tables, and decision trees. The document contains an extensive bibliography (in ANSI format) to support certain main points that are supplemented by information supplied by the Institute for Computer Sciences and Technology (ICST) resources. Additional information and verification was received from private interviews with various U.S. technical experts and equipment manufacturers.
\end{abstract}

Key Words: Beam-addressables; cartridges; cassettes; core memories; disks; drums; floppy disks; laser beams; magnetic bubbles; masstapes; solid-state memories; video disks.

\title{
I. Introduction to Computer Peripheral Memory Systems
}

Computer Peripheral Memory Systems are considered to be those principally auxiliary storage devices or subsystems that are a significant part of the computer system, but are logically separated from the mainframe. The mainframe is considered to usually include the Central Processing Units, or CPU(s), primary memory and closely coupled equipment such as Input/Output ( I/O) channel units.

Computer Peripheral memory systems generally supply data that is destined to be stored in the computer main memory. The peripheral memory systems supply large quantities of information at relatively slow rates to the computer. This information is then selectively routed to the computer internal memory, such as the main memory.

The peripheral memory hierarchy thus serves the computer with large quantities of information that the computer needs to fulfill its requirements. The peripheral memory function of thirteen types of such memories is presented in this report and their cost/performance characteristics are compared.

A. Categories

1. Rotating cylinder or disk memory systems a. Drums 
A magnetic drum is a cylinder-type device that is capable of being rotated rapidly. It is coated with a magnetic material in which bit patterns may be recorded and be read from. The bit patterns run in bands or tracks around the drum. A drum system may have many drums in it.

Read-write heads are positioned in rows near the drum surface. As the drum revolves, different parts of a track or band are read or written on by the associated read-write $(R / W)$ head. There is a separate $R / W$ head for each track. [HELSA 70]

Magnetic drums form part of the storage system, and serve as primary or secondary memory. A magnetic drum has a shorter access time than most magnetic disk systems; access time for drum memory is approximately one-sixth that of movable head disk memories. Magnetic drums are much faster in operation than magnetic tapes, and slower than magnetic cores.

\section{b. Disks}

The magnetic disk is one of the most widely used devices for direct access to data. The recording medium is a set of metal disks coated with magnetic material, such as gamma-ferric oxide. The disks are mounted on a rotating spindle. Data are recorded on tracks on the disk surfaces and are read or written as the disks rotate. The concept of disk storage is similar to that of a phonograph record except that the tracks are concentric instead of spiral. The stack of disks is referred to as a disk volume, and if the volume is removable, it is referred to as a disk pack. [KATZH 74]

Data are recorded on both surfaces of a disk (except the top and bottom surfaces of a volume that are used for protection, or perhaps servo information) and a single access arm controls two $R / W$ heads--one for the upper surface and one for the lower surface of each disk. (Some manufacturers use two or more heads per surface). The access arms form a comb-like assembly that moves in and out together. Usually, a single $\mathrm{R} / \mathrm{W}$ head is used to access a single surface, however some disks have a separate head assigned to each track. [KATZH 74]

The components that perform reading and writing are the magnetic core and winding of a head. The rest of the head is the mechanical assembly which supports these components at the correct position with respect to the disk surface and the electrical leads to access the windings. To write information on a disk, current is driven through the windings around the gapped core in the head. The current magnetizes the core which in turn magnetizes the magnetic material on the disk surface directly under the gap. Alternating the polarity of the current results in alternating the magnetization direction in the medium as the medium moves past the core. In reading the track of information, the same winding is involved. It senses previously written magnetic transitions in the disk and produces an output voltage across the winding. 
Each track can store the same number of bits and is identified by a track address. Each surface is identified by a head address (that is, the $\mathrm{R} / \mathrm{W}$ head used to read the surface). The heads are switched electronically when a given surface is to be read. Each track is identified by (and located by) a track number and a head number. A magnetic disk is read or written by first moving the access arms to the proper track address prior to the input or output operation and by then switching on the desired $R / W$ head. Disk seek refers to the process of moving the $R / W$ head to the proper place. The time necessary to retrieve information from disk storage is therefore a function of three variables: seek time, the time necessary to rotate to the desired position on a track, and data transfer time. [KATZH 74]

A disk storage module has three major components: the disk volume, the access arms and $R / W$ heads, and the disk mechanism that causes the recording surfaces to rotate and works in conjunction with the access arms and $R / W$ heads to record and retrieve data.

A disk storage unit is usually classified by several factors:

1. Whether the heads move or are fixed

2. Whether the disk volume is removable or not

3. Seek time and rotation speed (access time)

4. The number of disk storage modules per unit

5. The number of recording surfaces per module

6. The capacity of each track and the number of tracks per surface [KATZH 74]

(1) Moving-head disks

Hard disk systems are said to offer a highly cost-effective means of storing digital data. Hard disk systems consist of a continuously rotating magnetic medium and at least one magnetic head. Moving-head disk systems, especially, have dominated the peripheral, random-access data storage market and will probably continue to do so in the forseeable future.

In response to the requirement for the lowest possible cost in rigid disk devices, several manufacturers are marketing fixed disk, moving arm devices. Movable head disks provide large quantities of reliable low cost memory.

\section{(2) Stationary-head disks}

Stationary-head disks are sometimes called fixed-head disks or head-pertrack disks. When compared to other disks, fixed-head disks provide the fastest access times because the heads are permanently positioned above each track, therefore, seek time is zero. Since they have fewer moving parts they are also more reliable because many tight, mechanical tolerances are eliminated. The head-per-track disk ranks between floppy disks and moving-head disks when capacity is considered. 


\section{Tape storage memory systems}

Tape storage memory systems feature sequential-access. Sequentialaccess storage occurs when stored data becomes available only in serial sequence, regardless of whether or not all the information, or portions thereof, is desired.

The medium of the tape storage memory systems is a mylar tape coated on one side with a magnetizable material which may be magnetically altered in some way. It is the recording of data that magnetically orients the medium. The recording is nondestructive, this means that the orientation can be changed. Thus, the medium is erasable.

\section{a. Mass Tapes \\ 1. Magnetic 1.27 centimeter $(\mathrm{cm})$ wide tape}

The $1.27 \mathrm{~cm}$ wide tape has been the industry standard since 1953 when it was first commercially available. Magnetic tape units generally use reels of tape up to $26.67 \mathrm{~cm}$ (10.5 inch) in diameter, holding up to 732 meters (2400 feet) of $1.27 \mathrm{~cm}$ ( 0.5 inch) wide tape. As the mechanism of the tape drive causes the tape to move, data are recorded on and read from the tape as it moves past the magnetic $R / W$ heads.

The data are recorded on the tape in serial-by-character format in seven or nine tracks. On a nine track tape, there is an eight-bit character plus a parity bit for error detection which are recorded side by side on the nine tracks. On a seven track tape, there is a six-bit character plus a parity bit.

The capacity of each reel of tape is a function of the recording density, which is the number of characters recorded in a given length of tape. The range of the densities available is from $7.87 \mathrm{bpmm}$ (200 bpi) to $246 \mathrm{bpmm}$ (6250 bpi). A 7.87 bit-per-millimeter density means that 7.87 characters can be recorded in one millimeter of tape. In a 732 meter length of tape, the maximum range of unformatted capacity is from 5.76 to 180 million characters respectively at the density extremes specified previously. However, in practice, the data are recorded in variable length blocks with gaps of blank tape separating them for convenient buffering of the data blocks, thus providing easier data transfer. Therefore, this practice reduces the capacity of a tape to a lesser capacity, called formatted capacity.

\section{Magnetic tape greater than $1.27 \mathrm{~cm}$ wide}

We have surveyed four of the latest models of mass storage systems.

Mass storage systems combine the inexpensive cost of tape as a storage medium with the operating advantages of semi-random, on-line access.

There are two systems whose main objective is to utilize the short tape cartridge systems; this design improves access time by making the tape 
shorter and wider. In other words, the shorter and wider the tape, the better the access time.

To provide efficient search of information within a mass tape cartridge, the standard $1.27 \mathrm{~cm}$ tape was increased in width to $6.9 \mathrm{~cm}$ ( 2.7 inches) and decreased in length to $19.6 \mathrm{~m}$ (770 inches).

The third system is a fully automated on-line tape library for standard $1.27 \mathrm{~cm}$ magnetic tapes. All three systems have experienced from one hundred to a peak of three hundred cartridge or tape loads per hour. [THEID 78] Thus, its use is to eliminate manual tape mounting operations.

The fourth system takes advantage of videotape recording technology. It is very similar to the magnetic tape systems with the exception that it is written onto and read from magnetic $5.08 \mathrm{~cm}(2.0$ inch) wide by $1158.24 \mathrm{~m}(3,800$ feet $)$ long videotape.

Magnetic Media for Small Computer Systems

Since the initial introduction of magnetic media products for small computer systems, magnetic media products have grown and extended in the same direction as large-scale-integration circuit chips. Up until about 1973, the only magnetic media products widely used for small computer systems were the tape cassette and magnetic card. Then the $0.635 \mathrm{~cm}$ ( 0.25 inch) data tape cartridge was introduced, followed in rapid succession by the floppy disk, minicassette, minicartridge, and minifloppy disk. This proliferation of magnetic media has led to a wider choice of solutions for the equipment designer. In the case of tape magnetic storage systems, the designer can choose a magnetic medium to closely meet his requirements for storage capacity, access time, transfer data rate, and cost. [MANLA 77B]

These three types of removable-storage recording media are at the present well established -- tape cassettes, tape cartridges, and floppy disks. Each type has its own combination of performance characteristics. New designs are occurring constantly in each of these fields, and further developments are expected.

\section{b. Cartridge}

The term "tape cartridge" defines a device wherein the tape medium is enclosed in some type of container. In this container, there is either an endless unidirectional loop of tape on a single reel or a bidirectional tape on two co-planar take-up reels driven by an elastic belt drive. A typical tape cartridge contains $0.635 \mathrm{~cm}$ wide magnetic tape capable of recording $63 \mathrm{bpmm}$ (1600 bpi) (phase encoded) on up to 4 tracks. The speed of operation varies from manufacturer to manufacturer.

Even though the tape cartridge appeared on the market before the floppy disk, its impact has not been felt until recently. The storage capacity 
is approximately four times that of the cassette since the tape cartridge has four tracks instead of two and writes at twice the density or 126 flux transitions per millimeter (3200 flux transitions per inch) instead of $63 \mathrm{ftpmm}$ (1600 ftpi).

Recent technological advancements allow densities as high as $252 \mathrm{ftpmm}$ (6400 ftpi) which with double encoding give densities of 252 bpmm (6400 bpi). This yields data transfer rates as high as 192 Kbits per second in the serial mode with a storage capacity 16 times that of a standard cassette.

Minicartridge is the miniaturized version of the standard tape cartridge utilizing the same technology. It is about the same size as that of a standard cassette with a very small, inexpensive, and simple drive, nearly as low in cost as the minicassette drive; but the minicartridge cost is close to the cost of a standard tape cartridge.

Packaging in a sturdy, well built, compact data storage device as in the standard cartridges, confers reliability and high system performance. The unit cost is approximately twice that of a cassette even though the capacity is approximately one-half of the standard cassette. The minicartridge contains a $0.381 \mathrm{~cm}$ ( 0.15 inch) wide by $42.67 \mathrm{~m}$ (140 ft) long high-performance magnetic tape capable of recording $63 \mathrm{bpmm}$ on up to 2 tracks.

\section{c. Cassettes}

The cassette is a mature product and one of the earliest products from the class of specialized, removable, magnetic media for mass memory on minicomputers and microcomputers. The digital cassette has been on the market for about seven years and originally evolved from the audio variety to a reliable digital memory. The technology is well understood, including its advantages as well as its limitations.

Manufacturers claim that the level of reliability for the cassette is high with mean-time-between-failure (MTBF) as great as 15,000 hours; the encoded information, if reasonably protected from the environment, is reliable for over a year. Although many units use the simple mechanical drives typical of older audio cassette systems, there is a trend toward capstan and servo motor controlled drives, particularly as higher tape speeds and packing densities become more common. [CONWJ 77A]

Undergoing a "mini" revolution, the minicassette has reached the stage of proposed ANSI Standards. These minicassettes are used where size, weight, power consumption and cost are major considerations.

\section{d. Floppy disks}

"Floppy disk", "diskette", and "flexible disk" are several names for the low-capacity, low-cost answer to block-random-access storage for small computer systems. The name "floppy disk" is most commonly used. A floppy disk is in effect a large, circular piece of magnetic tape. Why 
was it called a floppy disk? Because its recording medium is a disk of oxide-coated mylar, unlike the rigid aluminum disks and drums which had been used in previous storage devices.

The major use of floppy disks is in data-entry systems; but intelligent terminals and remote-batch applications are not far behind. Blockrandom-access capability is the strongest attribute of the floppy disk. Random-access memory is a storage technique in which the time required to obtain information is independent of the location of the information most recently obtained. The storage system can access any piece of data on the floppy disk in less than one-half of a second.

\section{Optical data storage memory systems--laser beam}

Optical laser beam addressable memory techniques have been recognized as one alternative to conventional magnetic memory technology. These optical techniques for data storage have advanced significantly during the last decade. The introduction of optical techniques to computer mass memory applications is undergoing a cautious evolutionary process because of the lack of widespread commerical success in optical recording even though there are some unique devices that have been successfully demonstrated. Secondly, it is said that there is a continuous advancement in magnetic recording technology. Its projected performance improvement is of sufficient magnitude to satisfy the marketplace until at least 1980. Thirdly, even though the optical memory technology has proved technically feasible, some projections indicate that such systems are not yet economically competitive with existing mass memory technologies. [CHEND 75]

4. Solid-state peripheral memory systems

a. Charge-coupled devices

A charge-transfer device is a generic term that is applied to a family of several types of integrated circuit semiconductor devices, one of which is the charge-coupled device. A charge-coupled device (CCD) stores minority carriers in potential wells and transfers this charge as a packet by translating the potential minima parallel to the device surface. This operating characteristic can be used for developing large serial-block oriented solidstate memories. Since CCD, LSI memories are reported to be less costly to fabricate than conventional random-access semiconductor memories, several manufacturers have announced that they are developing CCD memories for applications such as fast-auxiliarymemories (FAMs) or peripheral mass memories. Because of their cost advantage and relatively faster operating speeds, CCD memories are found to compete strongly with drums and fixed-head disks in the rotating mass storage media.

\section{b. Magnetic bubble memories}

Magnetic bubble memories are currently fabricated from synthetic garnet materials. Magnetic cylindrical domains, located in a thin magnetic garnet film that is grown on a non-magnetic substrate, are capable of 
storing information for memory purposes. Thus, bubble memories differ vastly from semiconductor memories since these are currently fabricated for the most part from silicon materials. This basic material difference and high cost of initial product is said to have led to a generally low acceptance factor of this technology in the past. Bubble memories are reported to offer potentially higher information storage densities at extremely low-power operation when compared to any other $\mathrm{R} / \mathrm{W}$ memory technology. In addition, bubble memories also provide nonvolatile information storage. Recent developments, using these advantages, have started to break through the aforementioned resistance barrier. Based on these developments, several manufacturers are now offering magnetic bubble devices for use as information storage devices in terminals, point-of-sale equipment and other applications.

\section{c. Metal-oxide-semiconductor (MOS) memories}

MOS large-scale-integration (LSI), random-access (RAM) circuitry is currently found in many computer main frame memories as the basic storage element. This application was formerly completely dominated by core memory systems. Many of the MOS product manufacturers are reporting that their products are now displacing core memory in existing systems (as replacements) and in new designs. Moreover, recent advertisements have announced the introduction of MOS LSI RAM into another portion of the computer memory hierarchy - the peripheral mass storage level. Supporting articles justify this introduction since they claim that MOS memories are now less costly than before and are offering operating advantages, such as unconditional random-access and a nonrotating medium, not previously available at this memory level.

\section{Random-access magnetic memory system--Core memories}

Random-access magnetic core memories once accounted for nearly 100 percent of computer main frame storage. This memory technology depends on the magnetic properties of donut-shaped magnets for storing information. These core magnets are strung on several wires in order to gain electronic access to magnetically stored data.

Since core memories have been in existance longer than solid-state memories manufacturers of these devices are able to reduce their costs significantly each year by relying on accumulated past experience. In addition, progress in semiconductor LSI circuits has aided the further development and cost reduction of core memory systems. Where before many discrete components were required, now a single LSI chip on a core memory card often performs the entire required function. The advent of microprocessors is said to even further enhance core memory development.

However, say many core manufacturers, despite all of these improvements, solid-state memories have eroded the core memory market.

One of the latest core memory developments has occurred in what manufacturers call bulk core store. This new development has resulted in a number of products that are designed to function as mass storage systems 
on the computer peripheral level. Manufacturers of these units believe that the excellent data reliability and data retention of this equipment are advantages over most solid-state memories.

\section{Alternate Memory Systems \\ a. Videodisk memory systems}

Videodisk memory systems can record and transmit data in various formats. At present, these memory systems are mostly directed towards the television video-recorder/playback market, hence their name of videodisk systems.

A few manufacturers are developing videodisk systems for computer mass memory applications -- this application is addressed in the contents of this forecast.

Information contained in a videodisk system is found to exist in various forms (both analog and digital) and is embedded within the material of a disk not unlike a phonograph record. Most of these devices are optical write and optical read systems; however, some of these systems rely on mechanical, contact readout devices like capacitance, pressure, or magnetic stylus-like pickups.

The amount of information that can be packed into a videodisk is as tonishing. This information packing density is achieved by making use of advanced fabrication tools such as laser or electron-beam systems. As a result, the videodisk bit size is allowed to shrink to about 1.5 square micrometers (at an estimated error rate of 1 in 10 billion).

The single disk storage capacity of this read-only memory (ROM) technology is reported to be about 10 billion bits. One manufacturer believes that future system specifications of a quadrillion bits of storage, data rates of 50 megabits per second, and information access times of tens of milliseconds are possible. According to researchers, such a system because of its high data transfer should improve the processing data rate of most computers significantly while at the same time data reliability is said to be strongly enhanced.

\section{b. Beam Addressable Memory Systems}

Electron beam technology, which dates back to first-generation computer memories, is currently again considered by several companies as a promising technology for fast mass storage. Sometimes called EBAM (electron bear addressed memory), Beam Access, or EBEAM (electron beam addressable metal-oxide-semiconductor), this type of memory system relies on information storage in MOS chips placed inside and near the face of a. cathode-ray tube. The tube's electron beam is used to both write into and produce data from the MOS chips.

Electron beam memories have advanced significantly due to major progress in areas such as MOS technology, high voltage power regulation, and analog-to- digital (AD) converters. This memory combines large data 
capacity, relatively fast access times and high data transfer rates with long-term retentivity; storage of data is rated in months without application of power.

According to manufacturers, electron beam memories are cost-effective over core and semiconductor memories when large amounts of storage (over 30 billion bits per module) capacity is required. Electron beam memories are attractive when used as fast auxiliary or peripheral mass storage memory in computer systems.

\section{Development--Parameters of the Various Peripheral Memory Systems}

The following sections provide a comparison of various peripheral memory systems with associated specific parameters. The systems and their parameters are shown in tables each of which covers a specific memory technology.

Certain yardsticks have evolved whereby one attempts to measure the merit of any given peripheral memory device or system. Capacity, access time, data transfer rate, and cost per bit are the basic performance characteristics of mass storage devices. Each mass storage unit has its own particular attributes, and many applications require that a hierarchy or mix of devices be connected in the same computer system. The short access times and high data rates of fixed head drums or disks save on main memory size, thereby reducing systems cost. However, the relatively higher cost per bit of these devices generally makes them too expensive for file storage, and movable head disk structures of higher capacity (and lower cost per bit) but longer access time are used. Generally, the lower level in a memory hierarchy includes tape for the archives and for data whose processing can be well scheduled in advance since further economies in cost of storage are possible at the expense of even slower access times. When designing or selecting a peripheral memory system, all these parameters and their effects on the system application must be considered. [HOAGA 72]

Following are some general definitions for these performance characteristics:

Formatted storage capacity - the net amount of storage available to the user excluding reserved space for sectoring and other overhead storage requirements expressed in megabits.

Unformatted storage capacity - the total amount of space on the medium for storing data with no reserved space for data organization, as for distinguishing between blocks of data.

Transfer rate - the speed at which data may be read from the unit in bits per second exclusive of seek and latency delays.

Average access time - the average period required to make the unit ready to address a specified memory location. 
Price per bit - this value is obtained by dividing the price of the device by the unformatted capacity of the device, where this capacity is expressed in bits. The prices used are End User prices. If End User prices are not available, Original Equipment Manufacturer (OEM) prices are used and flagged according to the information available.

Before a prospective buyer of peripheral memories makes a commitment, he should ascertain how the price in cents per bit was derived. Obviously, there are at least three methods that could be used:

a. The device alone - the price of the device divided by the capacity of the device in bits.

b. The device and its associated controller - the sum of the price of the device and the price of the controller divided by the capacity of the device in bits.

c. The controller and the maximum number of peripheral memory devices that can be connected to it - the sum of the price of the controller and the prices of all the individual memory devices that can be connected to it divided by the total capacity of all the memory devices.

Depending upon the given circumstances and the method used, one can obtain surprisingly different results.

\section{Drums and Disks}

A little elaboration on some of the definitions for performance characteristics as they apply to drums and disks follows:

Storage capacity - ordinarily the capacity would be expressed in eight bit bytes for drums and disks, but in this report we are using bits to make comparison.with other memory devices easier. In those cases where our sources had expressed the capacity of a device in bytes, we merely multiplied by eight to get the capacity in bits.

Also, for the sake of comparison, we use unformatted capacity where possible. In those cases where unformatted capacity is not available, formatted capacity will be provided and flagged as such. For a given device, formatted capacity will always be somewhat less than unformatted capacity because of reductions due to overhead, such as error correction codes, etc.

Average access time for the drum or fixed-head disk - average access time is calculated differently for different technologies: In this case it refers to the period required to rotate a selected section of the drum or disk track into position under the appropriate head. This length of time is called latency time. On the average, latency is one-half the time required for one revolution.

Average access time for the moving-head disk - refers to the interval the moving head requires to shift into position over the appropriate track and to begin transmitting information. This time is the sum of 
the latency time and the average head movement time. Average head movement time is the sum of all move times divided by the number of moves. It generally equals the time to move across one-third of the tracks. [DATPR 77B] However, some manufacturers define average access time as the time to do all possible seeks divided by the number of all possible seeks; they do not include latency time. In providing the figures in this forecast we have done our best to follow the first of the two definitions.

Data transfer rate - is a function of the packing density on the track and the rotational speed of the drum or disk.

Price (per bit) - this value is obtained by dividing the price of the device by the capacity of the device, where the capacity is expressed in bits. In those cases where the only price available included the price of the controller we have used that price in our computation and flagged the result.

\section{a. Drums}

Parameters of some drums which appeared to be typical are listed in Table 01. One manufacturer said that his firm would produce a drum to the user's specifications, but the drums listed in Table 01 are offthe-shelf equipment. The most significant parameter of the drums, when compared to other rotating memories, is fast average access time. The capacities and average access times of these typical drums are quite similar to the same parameters for the stationary head disks shown later in Table 04.

The capacity of the typical drums listed in the table range from 33.6 to 114.4 megabits. Transfer rates range between 4 and 11 megabits per second and average access times range from 4.3 to 8.7 milliseconds. The price per bit covers a wide range, from 22 to 560 millicents per bit.

\section{b. Moving-head disks (fixed media)}

Table 02 contains the parameters for several typical moving head disks that have fixed media. The disks that are listed are currently in production and being marketed to the end-user by the manufacturer. When comparing this category of disk with others, one notices the outstanding feature to be capacity that is larger than the other disks. From reading open literature and talking to manufacturers, it has been determined that the approximate capacity range for this category of disk to be 1640 megabits to 2540 megabits The most popular disk in this category has a capacity of about 2400 megabits per spindle. Often two spindles are in one unit, thereby providing a total capacity of about 4800 megabits per unit.

Numerous manufacturers were asked for the ranges of the parameters for this type of disk. Transfer rates for this type of disk have an approximate range of 1.5 to 38.7 megabits per second. Average access time 


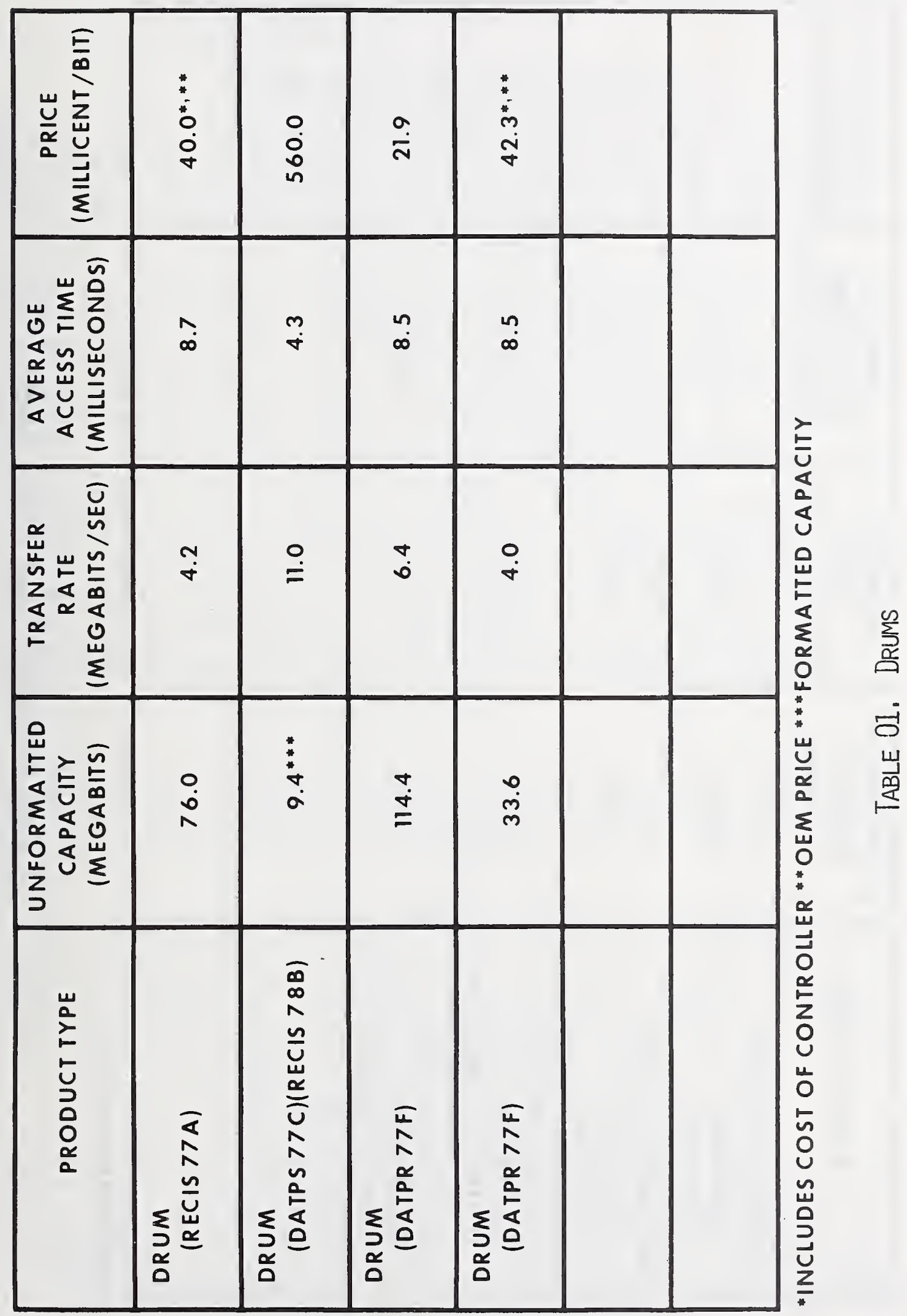




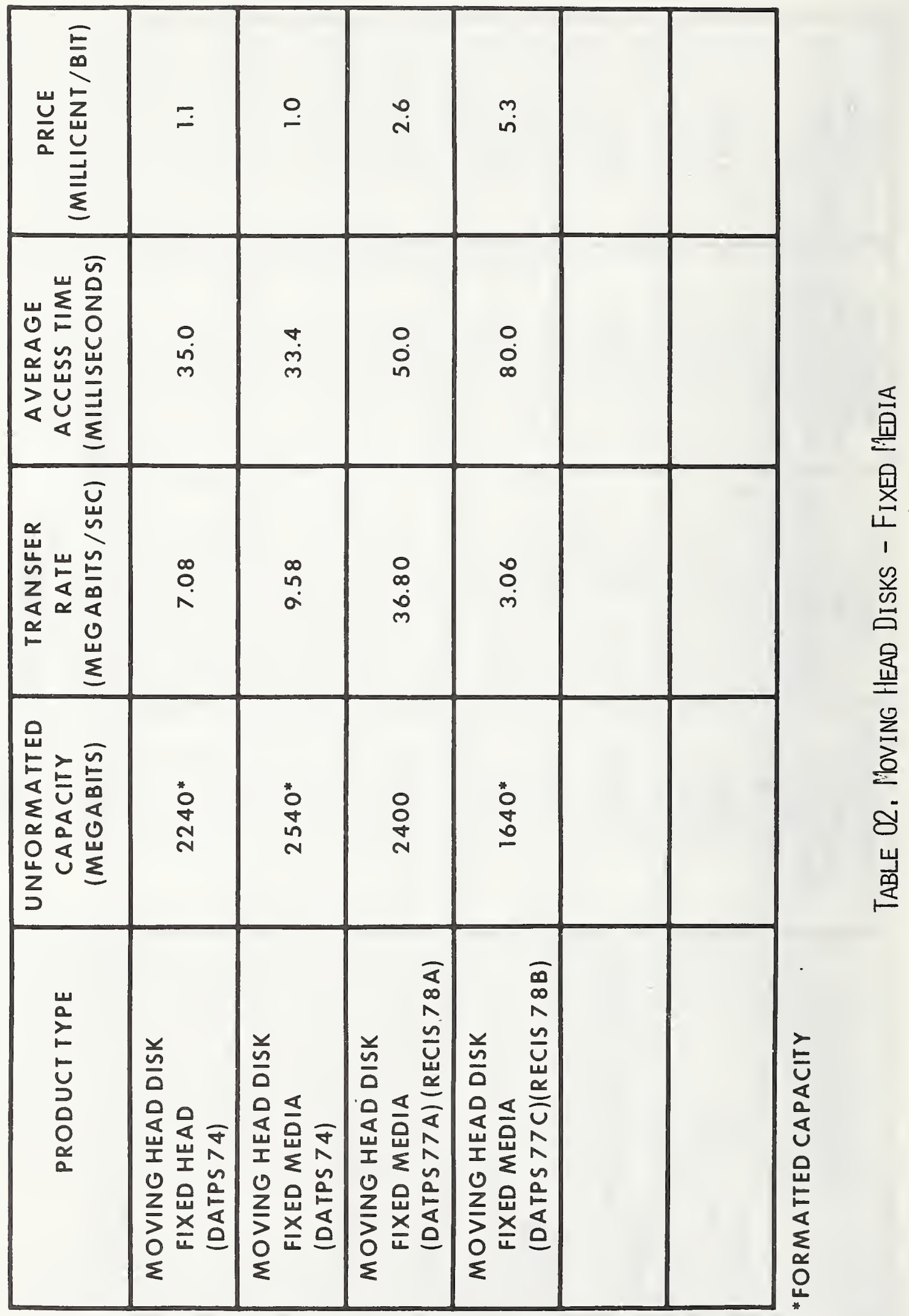


ranges between 33 and 269 milliseconds. Cost to the end-user is approximately 1.5 millicents per bit.

\section{c. Moving-head disks (removeable media)}

Several typical moving-head disks with removeable media (removeable packs) are listed in Table 03. These disks are currently in production and being marketed to the end-user by the manufacturers. These disks with removeable packs usually have less capacity and cost more per bit than the moving head disks with fixed media.

Numerous manufacturers were asked for the ranges of the parameters for this type of disk. Indications are that moving-head disks with removeable packs have an approximate range of capacity of 4.9 to 300 megabytes. The range of the transfer rate is 1.2 to 9.7 megabits per second. Average access time ranges between 30 and 135 milliseconds. End-user prices of this category of disk when supplied by a computer manufacturer are in the area of 2 to 20 millicents per bit.

Magnetic disks have offered large-capacity on-line R/W storage with relatively low cost and reasonable speed as compared to other memory devices. Disk files have become the dominant large-capacity storage medium due to its low cost, which is achieved through the large number of bits in the inexpensive storage medium sharing the expensive mechanical drive, servomechanism, channel electronics, control units, and other equipment. The large number of bits on platters mounted on the same spindle is made possible by high storage density. [CHANH 75]

\section{d. Stationary-head disks}

Table 04 lists the parameters for several stationary-head disks. All these disks are currently being manufactuuered. The last disk listed is sold in the OEM (Original Equipment Manufacturer) market only. The most outstanding feature of disks in this category is fast average access time.

Ranges of the parameters for this type of disk were obtained from numerous manufacturers. Our studies indicate the range of average access time to be approximately 2.5 to 17.0 milliseconds for disks of this category. Generally, capacities are in the range of 4.0 to 76.8 megabits. Transfer rates are between 4.1 and 8.8 megabits per second. The price per bit is in the area of 180 millicents.

\section{e. Cartridge-disks}

Parameters of several typical cartridge-disks are shown in Table 05. Generally, these disks are of relatively low capacity and slow average access time. The last disk entered in the table, besides having a removeable cartridge, also incorporates a fixed platter. Its capacity is much higher and its unit price per bit is much lower than the pure cartridge-disks we looked at. There are many such cartridge-disks (with 


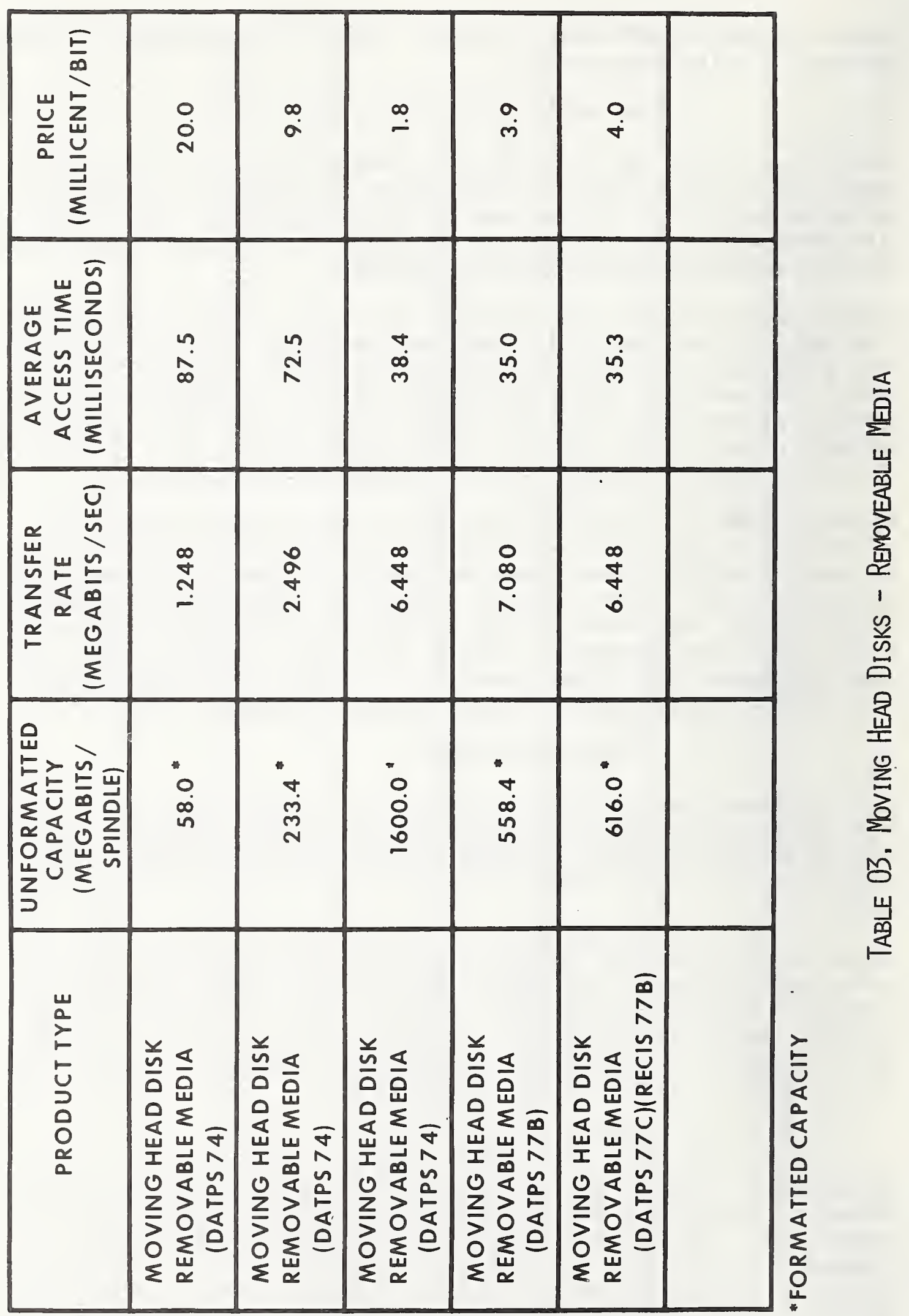




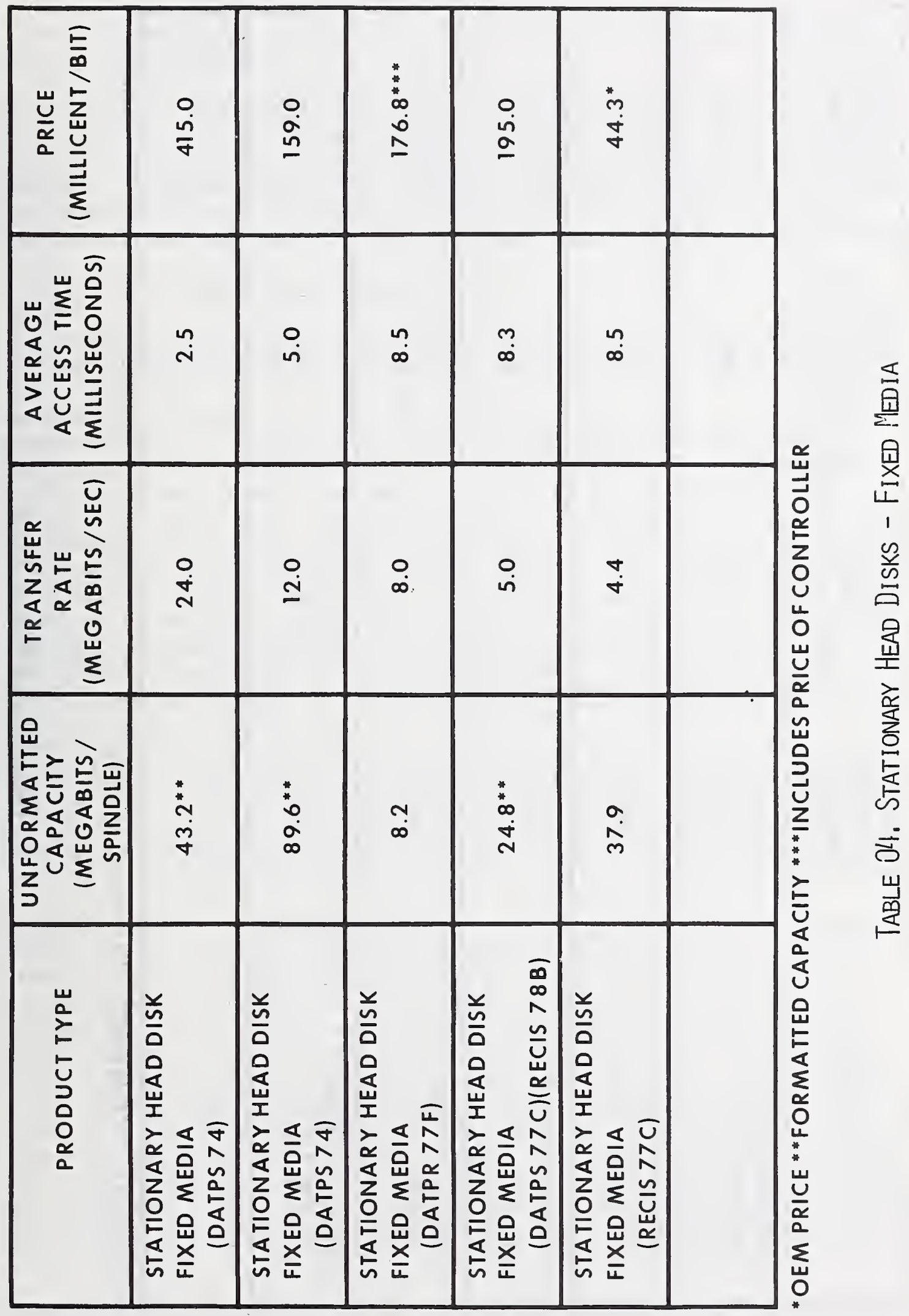




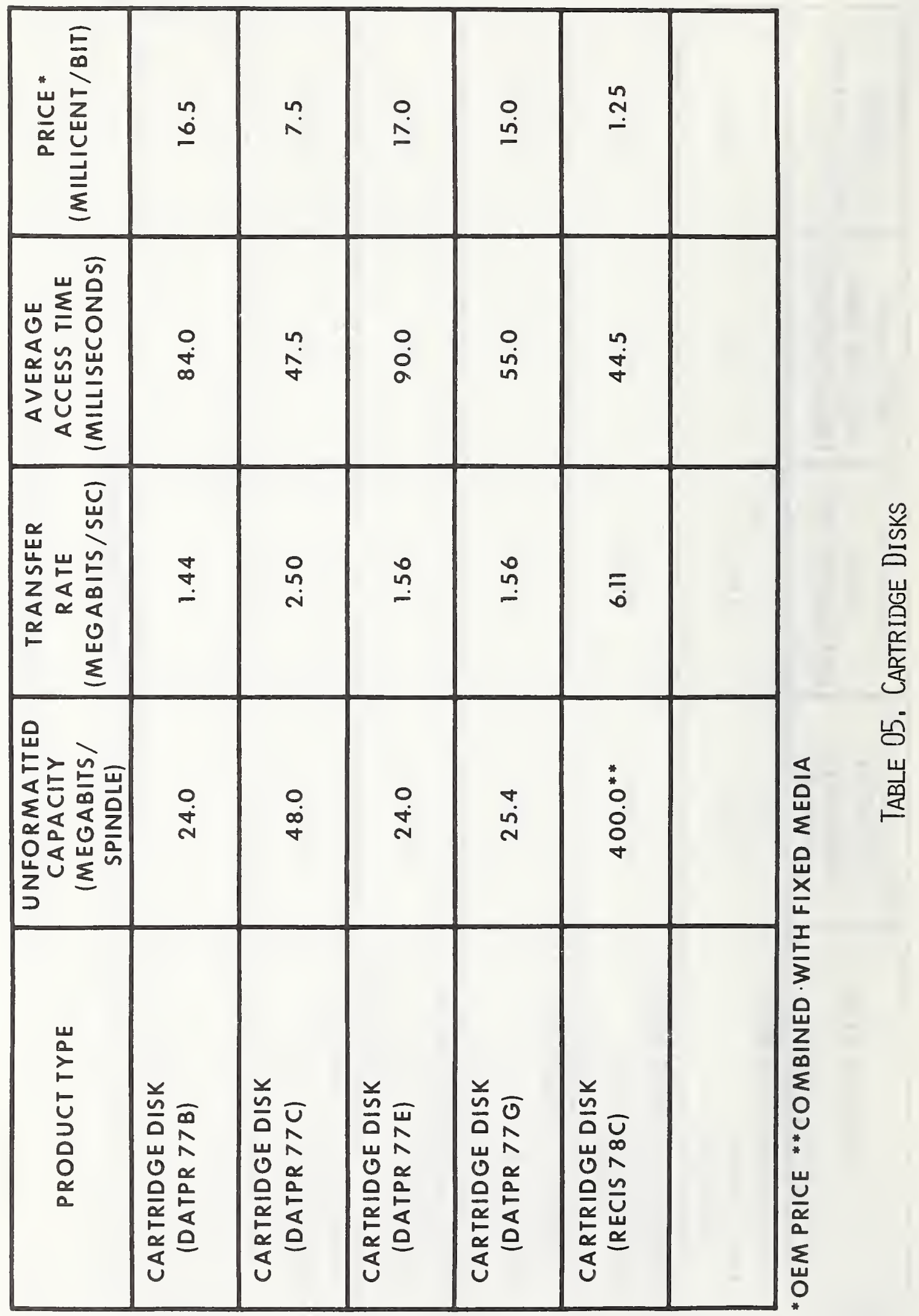


fixed and removeable platters) available in the market place, but most have a lower capacity.

After making inquiries to numerous manufactuers, we obtained the range of parameters for cartridge-disks. Disregarding the last entry in the table, capacities for the cartridge-disk ranged between 19.2 and 50.4 megabits, transfer rates ranged between 1.44 and 5 megabits per second, and average access times ranged between 41.5 to 90 milliseconds.

Generally, the price per bit fell between 7 and 17 millicents.

\section{Tape mass storage systems}

The following definitions are provided to give a better understanding of the performance characteristics of the magnetic tape storage system.

Unformatted storage capacity - The unformatted storage capacity of magnetic tape is a function of the recording density, which is the number of bytes recorded in a given length of tape. Densities are available from 8 bpmm (which means 8 bits can be recorded on a single track in one millimeter length of tape) up to $246 \mathrm{bpmm}$. In 731.5 meters of magnetic tape, the maximum storage capacity is 5.8 million to 180 million bytes when written at these densities. In reality, the data is recorded in variable length blocks with interrecord gaps of blank tape separating the blocks for easier access. This reduces the maximum capacity of magnetic tape and is called the formatted storage capacity. The total storage capacity of various magnetic storage media varies with block length and block length also affects access time.

Average access time - The average access time is the average time interval between the instant at which information is called for storage and the instant at which delivery is completed. If you access a large data file on tape, you can assume, according to manufacturers, that average access time to any data block equals half of the time required to read through the entire file.

Transfer rate - The transfer rate is directly proportional to the $\mathrm{R} / \mathrm{W}$ speed. It is the product of that quantity and the drive's recording density. The read/write speeds usually vary with the type of drive mechanism employed. The transfer rate comparisons can be deceptive because of the fact that data is always written in a blocked format containing gaps of blank tape between blocks. These inter-record gaps must be long enough to allow the tape drive to stop and start between data blocks. With high density parallel recording, convenient block sizes turn out to be much shorter than interrecord gap lengths, so the tape consists primarily of gaps. Thus, this not only reduces the storage capacity but also lowers the effective transfer rate. Since no data are stored in a gap, the effective transfer rate of blocks of parallel data can be only a small fraction of the theoretical maximum transfer rate.

Price - The price per bit for the tape storage system was established by dividing the advertised price of the system by the unformatted capacity. 
Table 06 shows the different ways these mass storage systems combine the inexpensive cost of tape as a storage medium with the operating advantages of semi-random on-line access. This table compares the characteristics of the four latest kinds of mass storage systems that were surveyed. The lowest price per bit of the four systems is an automatic tape library mass storage system with 29 microcents. However, it is not the fastest in terms of average access time. The mass tape cartridge storage system has an average access time of seven seconds which is the average time to receive the data cartridge and to deliver it to the $\mathrm{R} / \mathrm{W}$ station. As opposed to the other mass tape cartridge storage systems, the average access time is 15 seconds which is 8 seconds slower. One way to improve access time is to utilize shorter and wider tape. [THEID 78] In term of cents per bit of the two systems, the difference is 70 microcents which is the price paid for shorter access time with 0.35 terabits less than the other mass tape cartridge system. The video tape mass storage system has an unformatted capacity of 2.86 terabits at a price of 40 microcents per bit.

\section{Cartridges and minicartridges}

The previous definitions for mass storage systems hold true for the tape cartridge systems; however, the densities are different.

The densities of the cartridge systems are available from 63 bpmm (which means 63 bits can be recorded in one millimeter length of tape on a single track) up to $252 \mathrm{bpmm}(6400 \mathrm{bpi})$. [MANLA 77C] The maximum storage capacity, at these densities, on 91.4 meters of cartridge tape is 5.8 (using just one track) to 23 (using 4 tracks) million bits.

Table 07 shows the characteristics of the tape cartridge and mini cartridge. The typical standard size cartridge has an unformatted storage capacity of 23 million bits with a transfer rate of 48 kilobits per second. The average time is 20 seconds. The price per bit ranges from 3.7 to 13 millicents depending upon the application requirements. The minicartridge unformatted storage capacity is from 2.7 to 6.2 megabits depending upon packing density and number of tracks. The minicartridge has up to 2 tracks whereas the standard cartridge has 4 tracks. The price per bit for the minicartridges ranges from 4 to 14 millicents.

\section{Cassettes and minicassettes}

The definitions in the previous mass storage systems hold true for the cassette systems; however, the range of density is different.

The densities of cassettes are available from 32 bpmm ( $800 \mathrm{bpi}$ ) (which means 32 bits can be recorded in on millimeter length of tape on a single track) up to $63 \mathrm{bpmm}$. The maximum storage capacity, at these densities, on 91.4 meters of cassette tape is between 2.9 megabits for single track and 5.8 megabits for a two-track unit. 


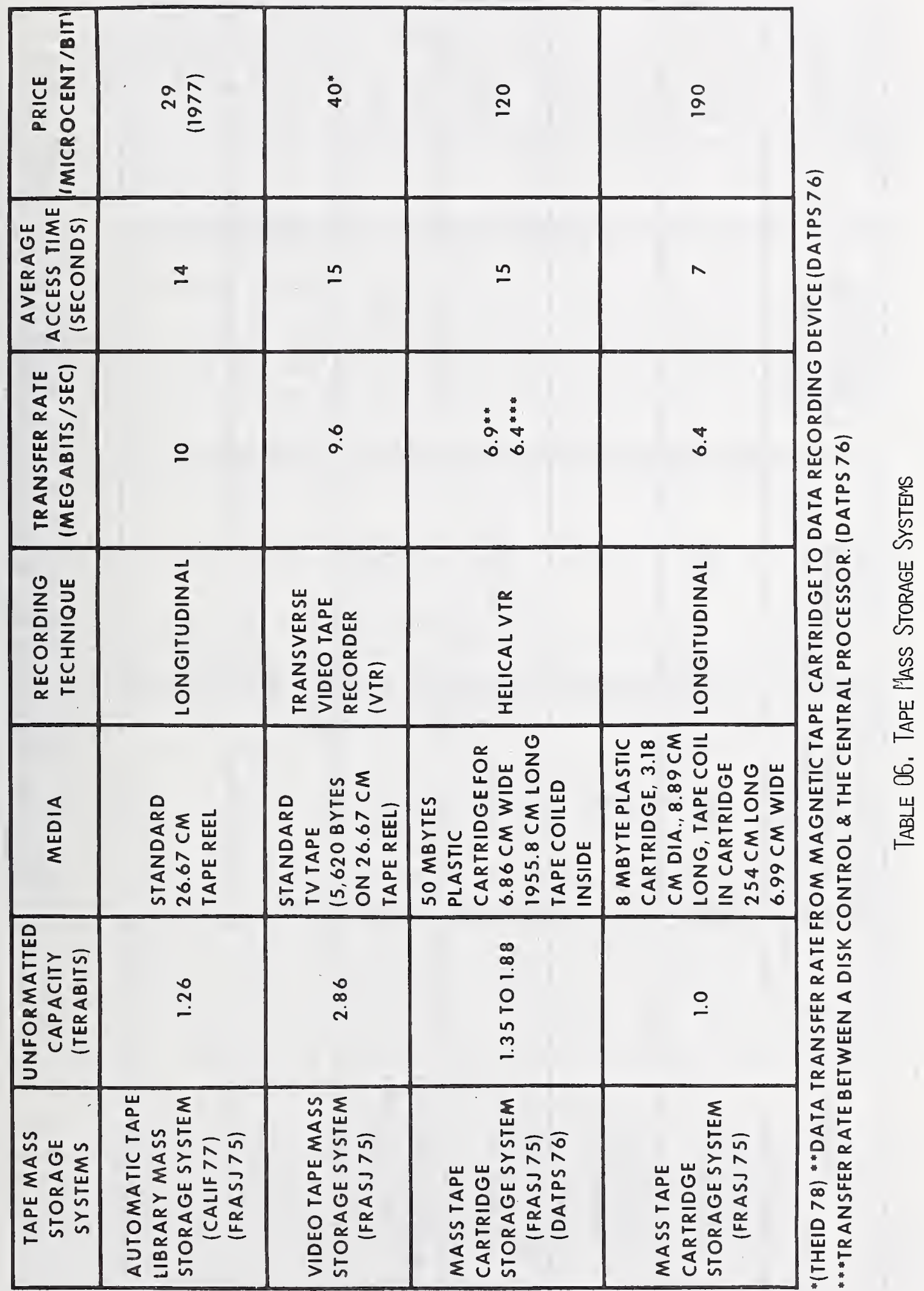




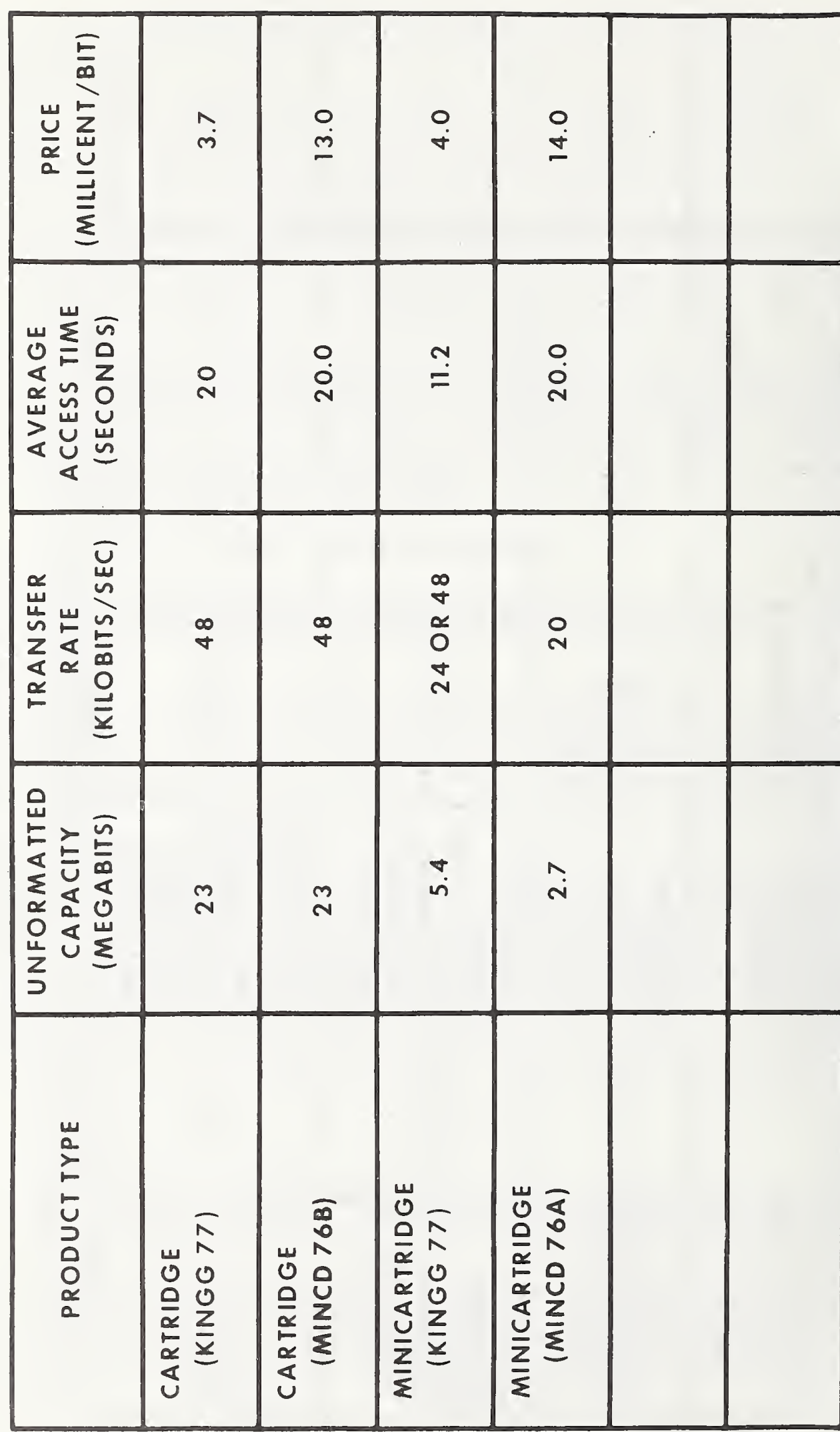

崫

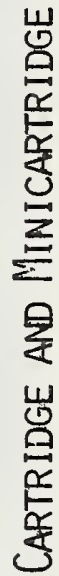

8

崖 
Table 08 shows the characteristics of the cassette and minicassette. The unformatted capacity of a typical cassette is 5.4 to 5.8 megabits. The typical transfer rates range from 8 to 24 kilobits per second. There are some cassette units that have transfer rates up to 60 kilobits per second. [CASWS 75C] The typical average access time is 20 seconds. Their price per bit ranges from 8.3 to 13.0 millicents depending upon the application.

\section{Floppy disks and minifloppy disks}

The following definitions are applicable to the floppy disk system.

Unformatted storage capacity - This is the total amount of space on the floppy disk for storing data without reserved space for data organization which is necessary for distinguishing between blocks of data.

Formatted storage capacity - This is the net amount of storage available to the user excluding reserved space for sectoring and other overhead storage requirements.

Transfer rate - This is the speed at which data may be read from the unit exclusive of seek and latency delays.

Average access time - The average access time is the average time in milliseconds required to make the unit ready to access a specified location on the floppy disk. By industry standards, average access time is computed by multiplying one-third the number of tracks by the trackto-track stepping rate and then adding head settle time and the average latency, which is one-half the rotation time. [CASWS 75A]

Price per bit - The price is established by dividing the advertised price of the system by the unformatted capacity.

The characteristics of the floppy disk and minifloppy disk systems are shown in Table 09. The typical single density floppy disk capacity is 3.2 megabits with the transfer rate of 0.25 megabits per second. The typical double density double sided floppy disk capacity is 6.4 megabits with a transfer rate of 0.50 megabits per second. The average access time for both single and double density floppy disks range from 162 to 286 milliseconds. The price per bit is 19 millicents for single density floppy disk. For double density floppy disk the price range per bit is 9.4 to 10.2 millicents.

The single density minifloppy disk capacity is 0.87 to 0.88 megabits with a transfer rate of 0.125 megabits per second. The range of average access time for single and double density floppy disk is 162 to 566 milliseconds. The double density minifloppy disks capacity is 1.8 megabits with 0.25 megabits per second transfer rate. The price per bit range for single density minifloppy is 4.4 to 45 millicents. For double density, the price per bit is 22 millicents. 


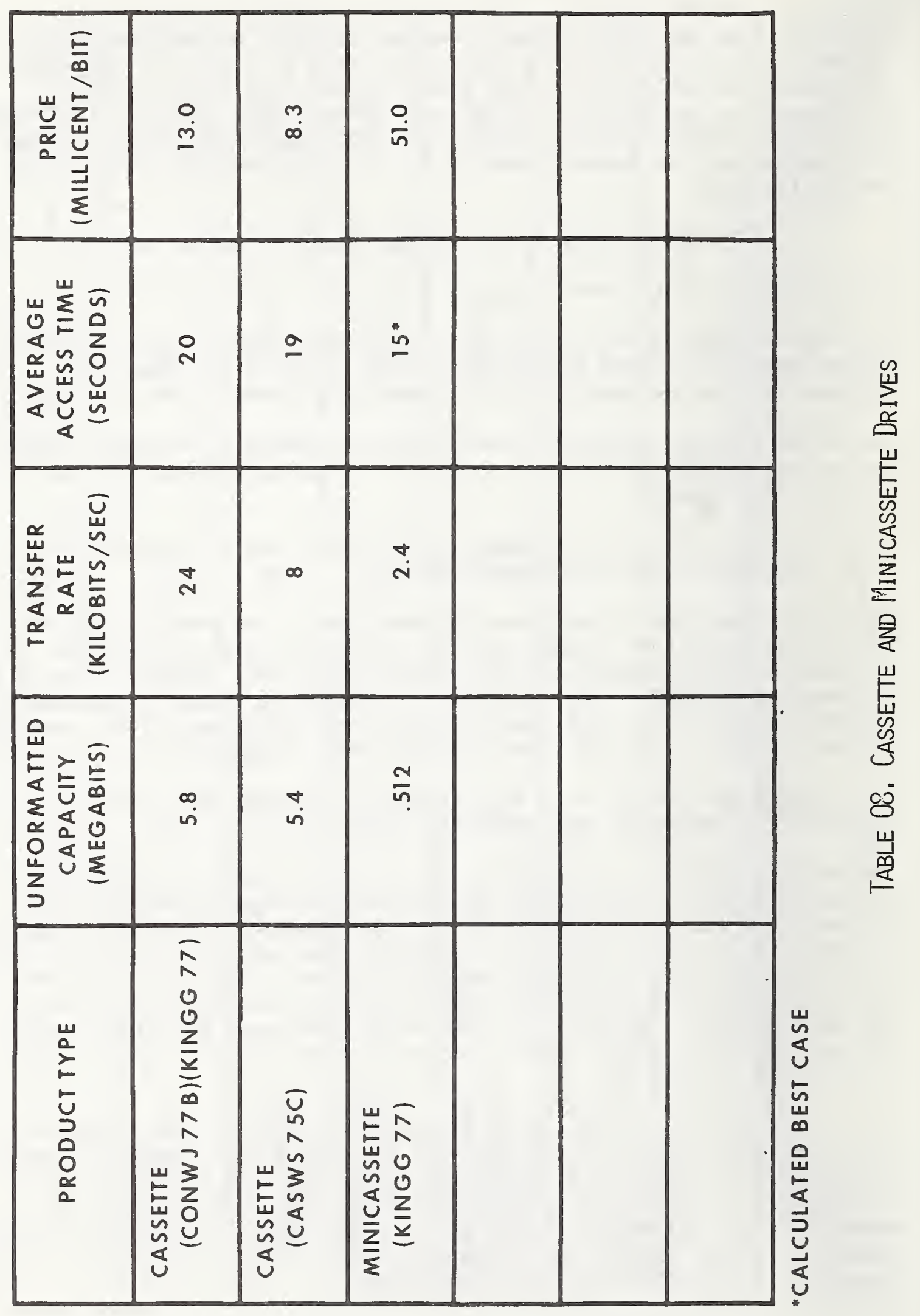




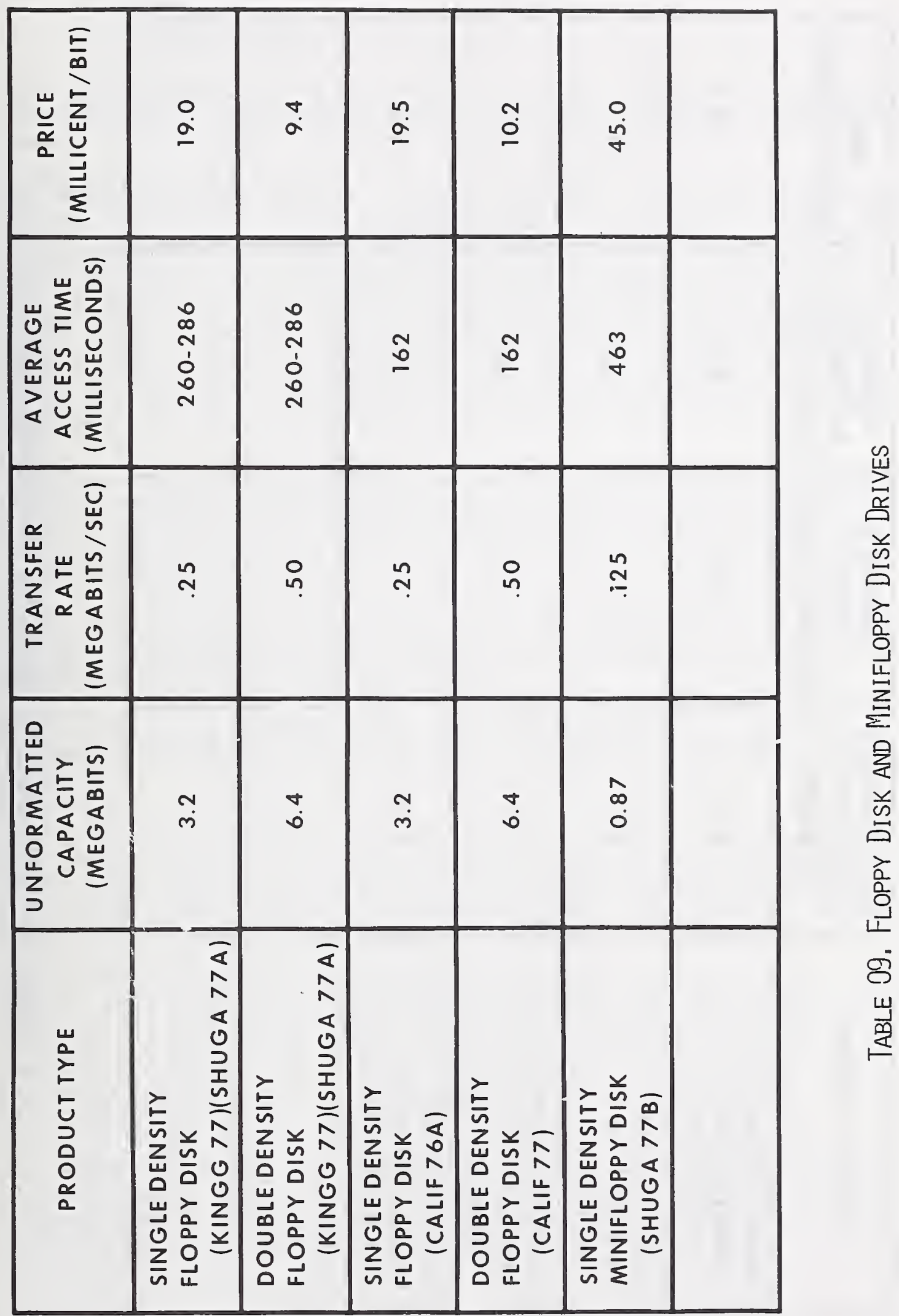




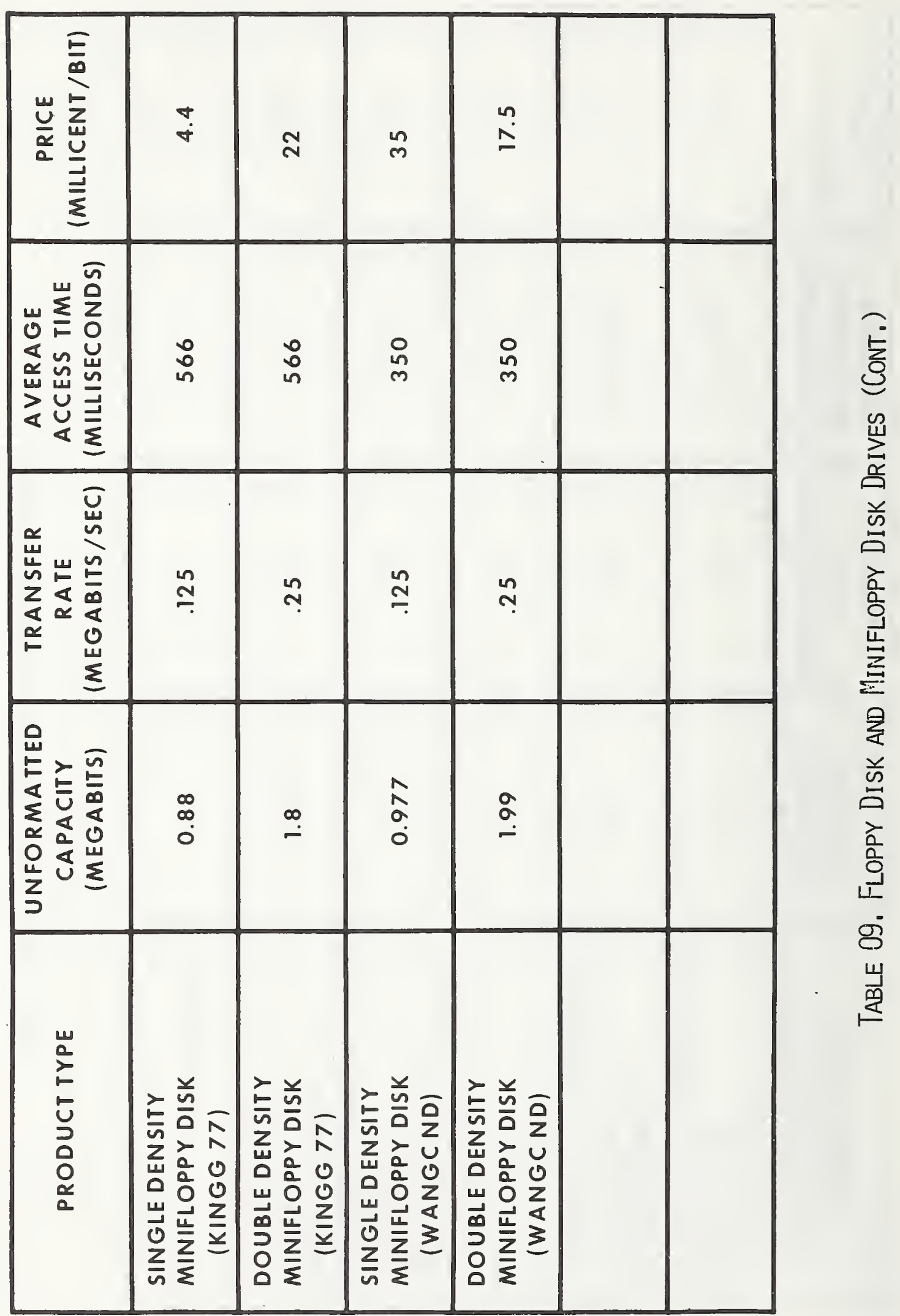




\section{Laser beam}

The following definitions are provided to give a better understanding of the performance characteristics of the optical laser mass memory systems.

Unformatted storage capacity - The information of the laser mass memory system is stored in a track-oriented format on metal film strips. Each strip contains 13,000 user tracks and each track can contain 15,385 eight-bit user bytes. The total formatted capacity per strip is 1.6 billion bits. In addition, there are approximately 37 percent more bits on the strip which are used for overhead functions such as preamble, error recovery, record markers, check code, etc. The data tracks are spaced 7.44 microns center-to-center and the data is recorded down the track in 3.6 micron diameter areas. The unformatted capacity per strip is aproximately 2.2 billion bits. [PI 77]

Transfer rate - The transfer rate of the laser mass memory is the speed at which data may be read from or written to the $R / W$ unit. The burst transfer rate is 400 kilobytes per second. The typical writing transfer rate is 320 kilobytes per second and the typical reading transfer rate is 300 kilobytes per second.

Average access time - Average access time is the average time interval between the instant at which information is called for storage and the instant at which delivery is completed.

Price - The price per bit of the laser mass memory system was established by dividing the advertised price of the system by the unformatted capacity.

Table 10 shows the characteristics of present as well as planned laser mass memory systems. At the present, the unformatted capacity of laser mass memory is 1 terabit with 3.2 megabits per second transfer rate. The average access time is less than 20 seconds. The price per bit for the present system is 360 microcents.

The potential archival laser mass memory system capacity will be from 2 terabits for single side glass slides with the metal strip embedded to 4 terabits for double side slides. The projected transfer rate is 4.75 megabits per second with an average access time of 10 seconds. The projected bit price for one side slides is 10 microcents and for double side slides is 5 microcents.

The potential library laser mass memory capacity will be 1000 terabits with the same or better transfer rate and average access time. The projected price per bit is 1 microcent which is one order of magnitude less than the previous single side price. 


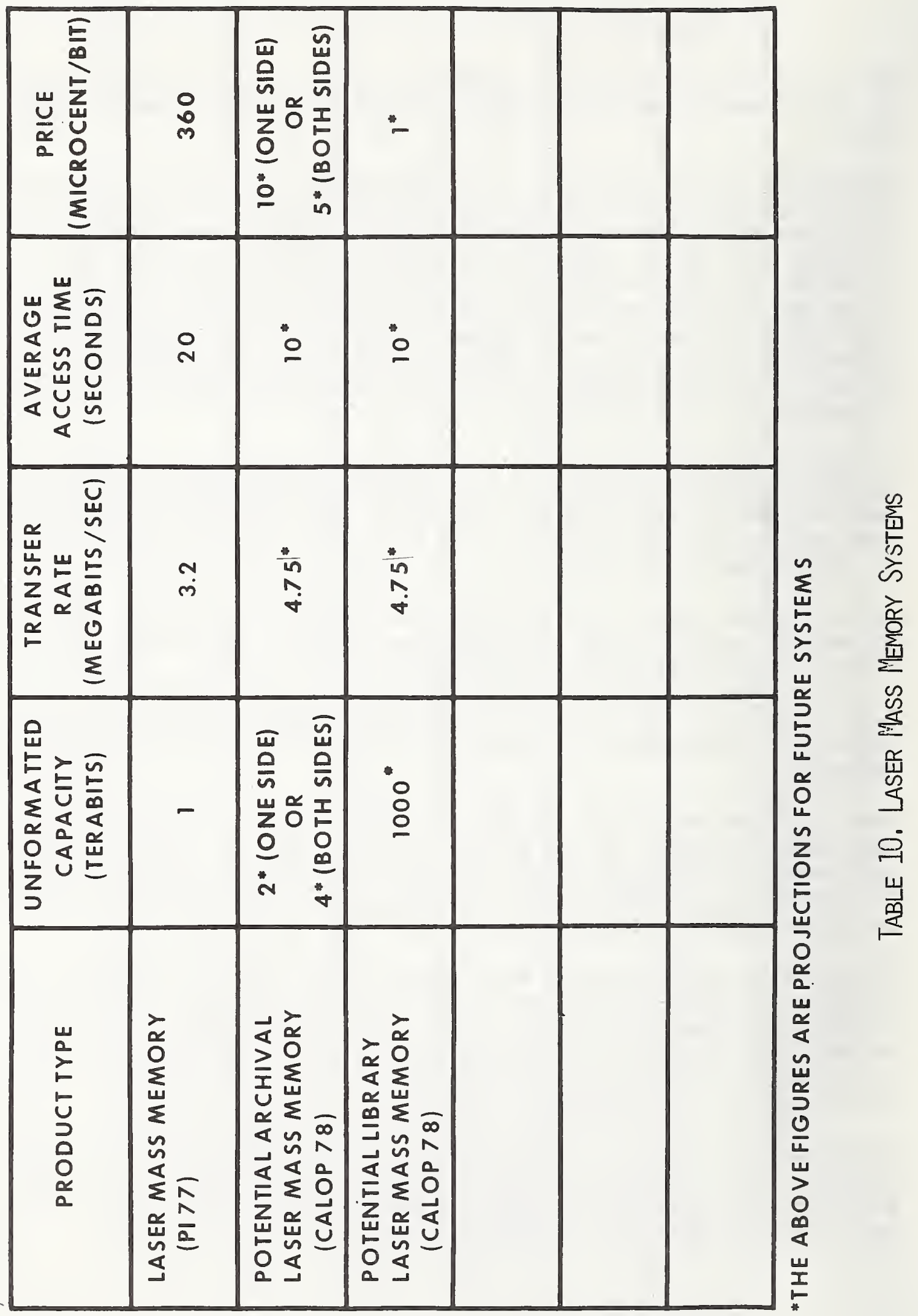




\section{Charge-Coupled Devices}

In order to provide a better understanding of the performance characteristics of charge-coupled device memories, some definitions that apply specifically to them follow:

Storage capacity - Various memory word lengths were found to be used in the different designs of this emerging memory technology. In each case, with word lengths ranging from 8 to 56 bits, the total memory word capacity was multiplied by the number of bits per word in order to arrive at the total bit-capacity of the memory as presented in the table.

Also, to make comparisons easier the unformatted capacity of each memory is presented in the table.

Average access time - For CCDs these parameters vary substantially from system to system. The reason is that CCDs are primarily serial devices and the organization of these structures has significant effects on access time. Generally, these memory systems allow the addressing of blocks, or lines, of data; after this data entity is addressed, a serial transmission of the data block, or line is performed. In this process, a certain number of cycles have to be performed serially to arrive at the correct address of the desired block of information.

Transfer rate - The transfer rate of CCDs is highly dependent on the speed of the individual memory chips and the structure in which these chips are arranged and addressed. Thus, if a CCD chip features a 10 megabit per second transfer rate and the chips are arranged to form 60-bit words (parallel operation), the transfer rate could be as high as 600 megabits per second.

Price - This particular parameter is obtained by dividing the advertised (or projected) price of the memory by its total capacity in bits. Care should be taken in order to obtain meaningful comparisons. For instance, in some cases the memory price could not be extracted from the price of the total computer system while in other cases electronics overhead, such as controllers, interfaces, etc., was considered part of the price. The most basic price per bit of the CCD can be found by dividing the chip capacity by the chip price. Many such values are presented in the table.

Table 11 shows typical designs of the CCD memory. One design, not truly a peripheral memory, is called the "file" memory system. In this system, the file memory is placed on a 1.25 megaword (56 bits per word) per second bus that interconnects both with the parallel and control processor of a new computer system. Each processor has its own fast bipolar random-access-memory (RAM) that can be loaded, upon request, by the file memory. The CCD file memory strongly enhances the numerical computation capability of the machine and allows 50 million floating point operations per second through "file" use. In this case, the cost/perfor- 


\begin{tabular}{|c|c|c|c|c|c|c|}
\hline 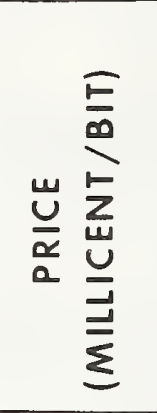 & 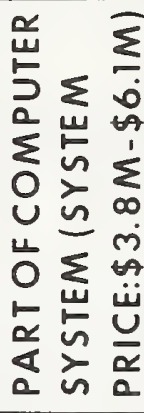 & $\begin{array}{l}0 \\
\text { W } \\
\text { N }\end{array}$ & in & $\begin{array}{l}0 \\
0 \\
\text { N }\end{array}$ & 品 & $\begin{array}{l}0 \\
\dot{\sigma} \\
\sigma\end{array}$ \\
\hline 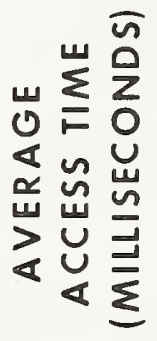 & $\begin{array}{l}8 \\
0 \\
\text { n }\end{array}$ & $\begin{array}{l}\text { o } \\
\text { in } \\
\text { N }\end{array}$ & $\stackrel{0}{0}$ & $\begin{array}{l}0 \\
0 \\
\text { 누 }\end{array}$ & $\begin{array}{l}8 \\
8 \\
\text { + }\end{array}$ & $\begin{array}{l}\text { 웅 } \\
\end{array}$ \\
\hline 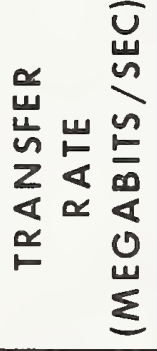 & $\begin{array}{l}0 \\
0 \\
0\end{array}$ & $\stackrel{N}{N}$ & in & $\forall$ & in & $\stackrel{0}{-}$ \\
\hline 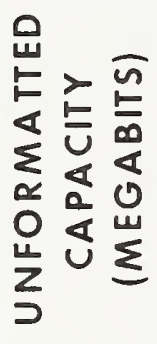 & $\begin{array}{l}\text { N } \\
\text { in } \\
\text { n' }\end{array}$ & $\dot{q}$ & $\begin{array}{l}+ \\
0 \\
0\end{array}$ & $\infty$ & $\begin{array}{l}\text { tे } \\
\text { o }\end{array}$ & $\underset{\infty}{+}$ \\
\hline 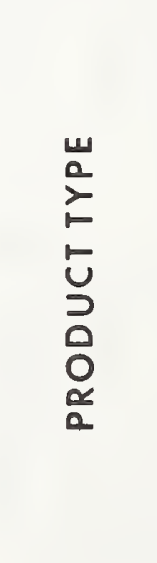 & 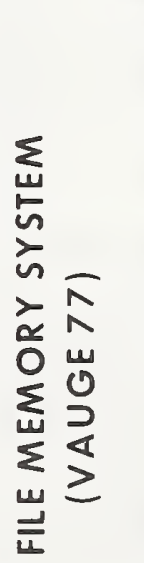 & 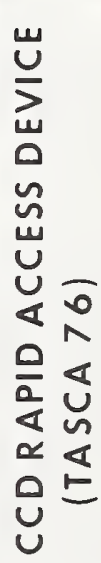 & 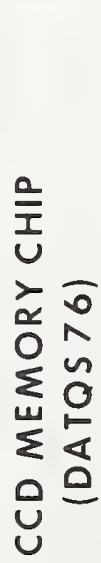 & 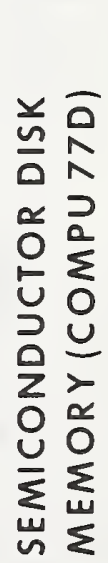 & 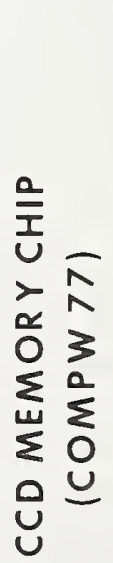 & 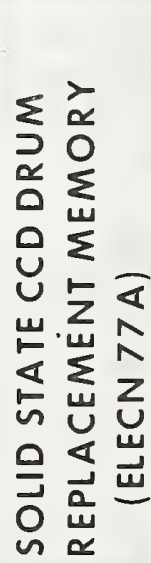 \\
\hline
\end{tabular}




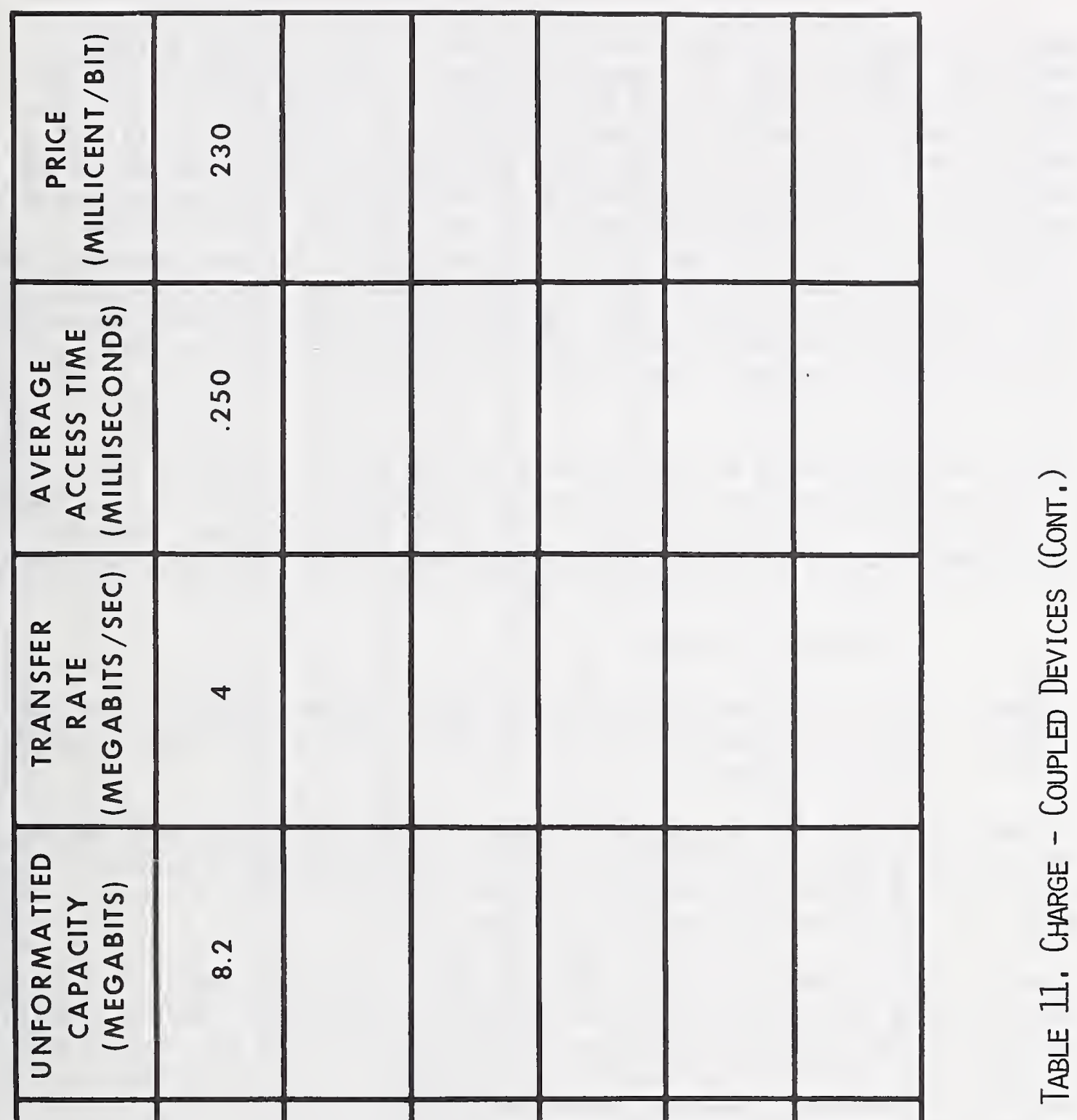


mance advantage of CCD implementation is realized since their cost is lower than bipolar RAM. CCD memory designs of Table 11 show capacities that range from 40 kilobits to 3,752 megabits. Oddly enough the higher capacity was not found in the peripheral but as an extension of the computer main memory. Transfer rates of 4 megabits to 600 megabits per second were encountered with the higher rate again in the nonperipheral application. CCD peripherals generally show transfer rates in the middle ten megahertz. Access times are comparable in these memories 100 to 500 microseconds. The smaller systems showed the shortest access time, indicating that larger systems used a more serial design approach; on the average, the table shows a price of 250 millicents per bit. A shorter access time generally results in a higher price per bit or as high as 940 millicents.

Clear cost/performance advantages were as yet not found in the CCD electronic disks designed as replacement units for magnetic disks. Only the CCD memory access time appeared better -- CCD electronic disk capacity was found to be less, price was higher, while data rates appeared to be about the same but are improving.

\section{Magnetic Bubbles}

In order to provide a better understanding of the performance characteristics of magnetic bubble (MB) memories some comments that apply specifically to Table 12 follow:

Storage capacity - The memory word length of the magnetic bubble memory was usually found to be 8 bits, or 1 byte. In each case, the total memory word capacity was multiplied by the number of bits per word to obtain the total memory capacity in terms of bits.

Magnetic bubbles (MBs), at this time, differ from other memories in that some of their memory is considered redundant. This means that a certain fraction of the MB memory is allowed to be defective to gain advantage in production yields -- an outside semiconductor, read-only-memory is necessary to identify, and thereby avoid these defects. In the accompanying tables, the unformatted capacity has been reduced by the redundancy factor and the parameter thus identifies only the number of operating, and directly available data bits of the memory.

Average access time - As with CCD's, magnetic bubble memory access times vary according to specific system design since these memories are also primarily serial in nature. Block random-access is common and again is quite similar to the CCD approach. At this time MB access times are still relatively slow since their shift rates, a primary factor in establishing access time, have not yet peaked out and should be improving shortly.

Transfer rate - Transfer rates of MBs are still being improved. The current speeds, according to researchers, are not yet representative of what this technology is capable of in the near future. Transfers are 


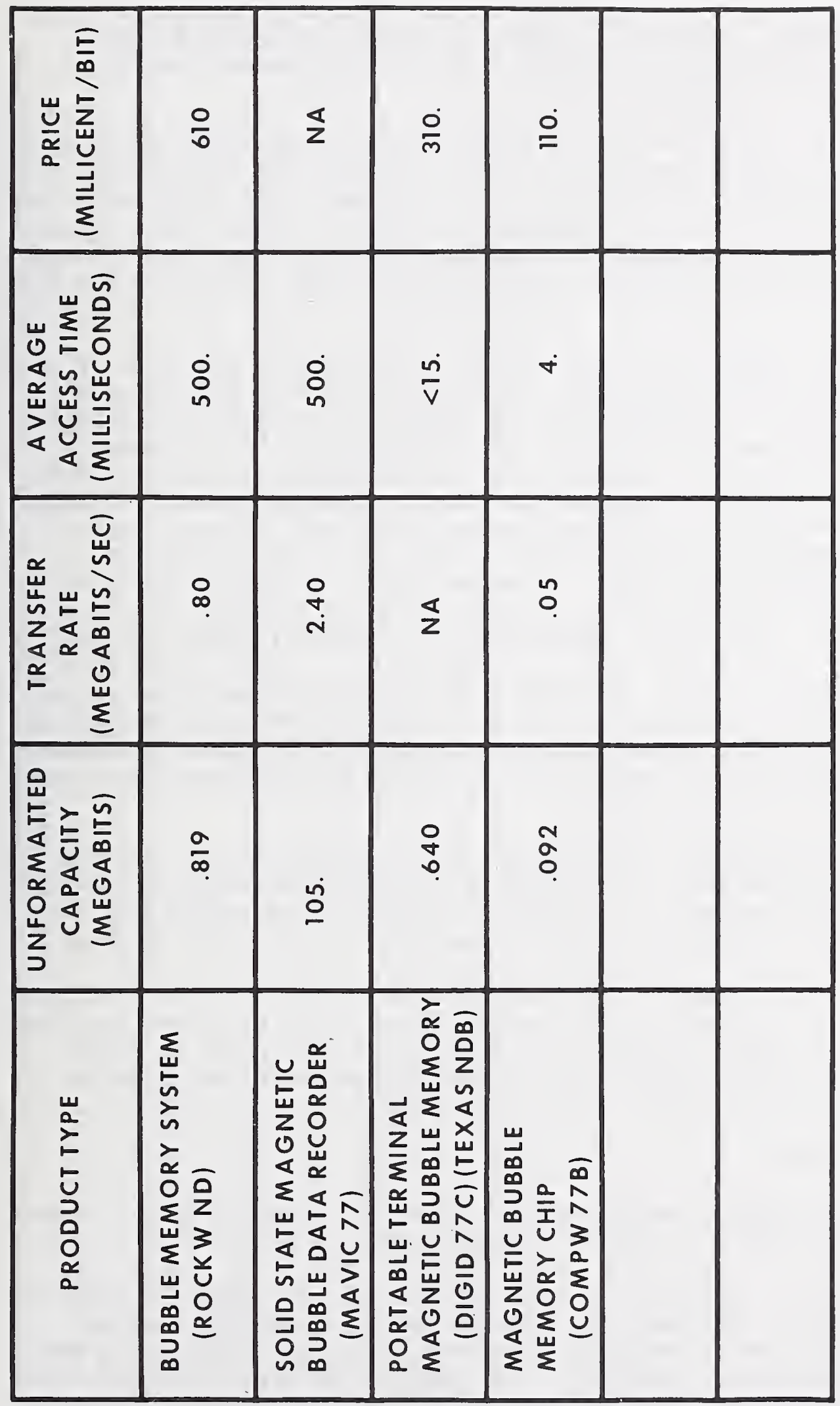

㟒 
serial in nature, like magnetic disks -- parallel transfer occurs by placing many bubble chips in parallel and then addressing and transferring their data simultaneously.

Price - The price per bit for the magnetic bubble memory was established by dividing the advertised price of each design by the unformatted capacity as indicated in the table. The most basic price of the bubble bit was found by using the single magnetic bubble package price (small quantities) and dividing it by its unformatted capacity. This value, when compared to other values in the table provides a "feel" of how much overhead cost is required for a system design.

Table 12 shows typical MB memory designs. As in CCDs, the capacity of these systems vary greatly and a range of 640 kilobits to 105 megabits was encountered. The larger capacity memories were generally employed in military systems while smaller capacities were found in commercial products. The range in transfer rates was 50 kilobits to 2.4 megabits per second. In this case, the difference in rates can be attributed to different products from separate manufacturers that are competing in this area. MB access time varied with the size of the system--the bigger the capacity the longer the access time. A range of 15 to 500 milliseconds was encountered. The price range as shown in the table appears rather chaotic and ranged from 110 to 620 millicents per bit.

The chip capacity of 92 kilobits is higher than any other solid-state chip commercially available at this time. Its low-end price per bit is also less than most LSI memory circuits although researchers feel that current values have not stablized because this garnet technology is new to the solid-state memory market.

Magnetic bubble memories, unlike other solid-state memories, are nonvolatile. Their magnetically stored data remains in memory without external supply of power. In addition, radiation exposure levels usually intolerable to semiconductor memories, have little effect on MBs .

In comparison to magnetic disks, magnetic bubbles at this time excel in access time according to Table 12. There is no comparison in either system capacity, data rate, or price per bit; however, rapid improvements are expected in these areas in the near future.

\section{MOS}

The following comments are presented in order to provide a better understanding of the MOS performance characteristics of Tabie 13.

Storage capacity - Metal-oxide-semiconductor memory designs of Table 13 are random-access memories. The inherent design, packaging, testing, etc. of these types of memories is more difficult and consequently more costly than most serial memories. For this reason large, peripheral MOS mass memories are not found and probably will not be available in the 


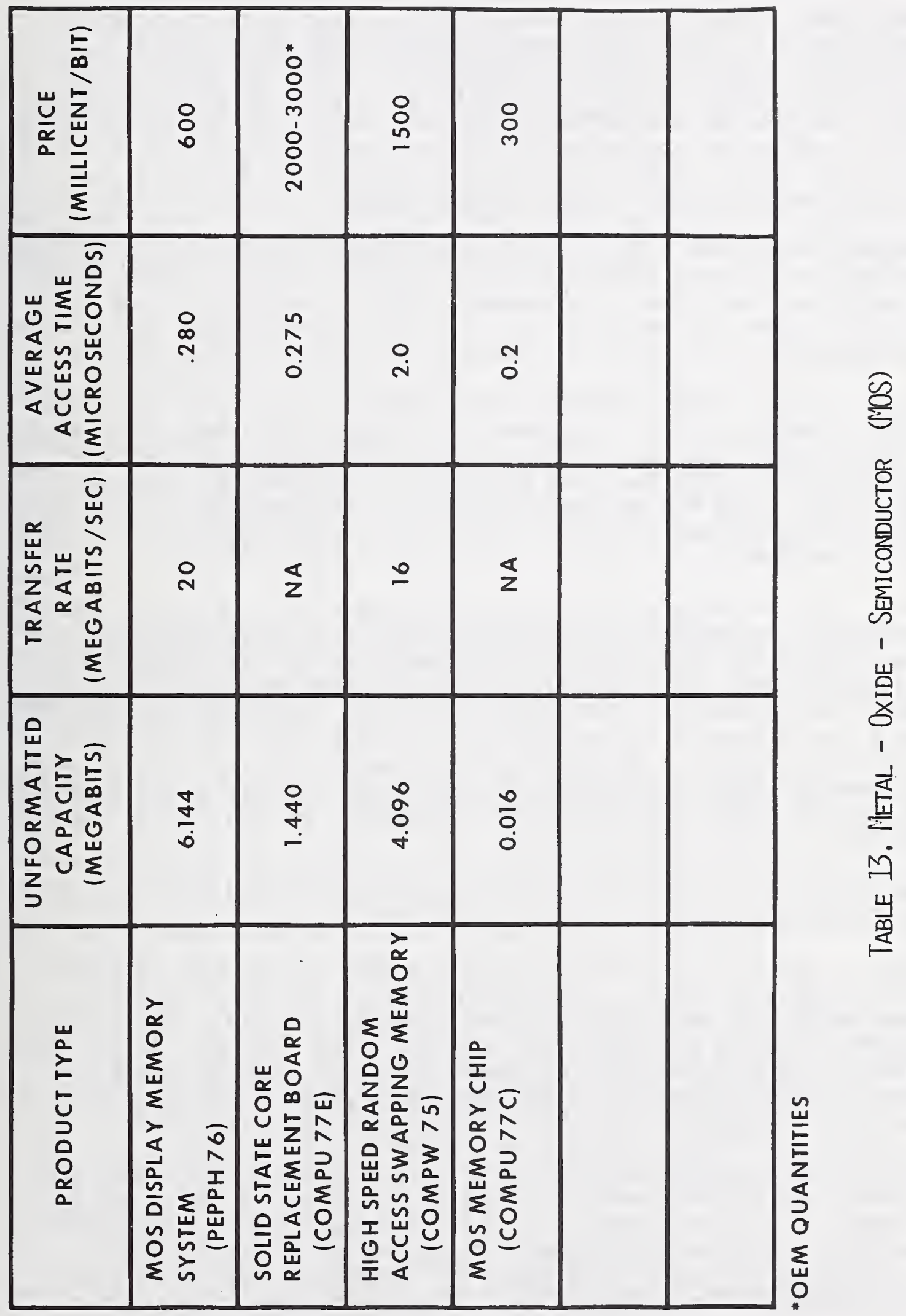


near future. The indicated unformatted capacity was found by multiplying the word, or byte, capacity of the particular design by the number of bits per word. Byte word lengths of 8, 16, 18, 22 bits or more were found to be common in the encountered MOS designs. It should be remembered that all the bits of the memory word are usually not available for data. Some memory system manufacturers revealed that as much as 5 bits of the 16 bit words might have to be used for memory data error corrections thus leaving 11 bits for data storage.

Average access time - The average access time of both static and dynamic MOS memories is much faster than other memories covered in this report. In addition, the access of MOS memory data is achieved in a completely random fashion. As indicated in the table, access times of hundreds of nanoseconds or less are quite common in this memory technology.

Transfer rates - The transfer rates of MOS memories can be relatively high, depending partly on the number of parallel MOS memory chips used in the design. This rate is also greatly enhanced since access and cycle times are very fast when compared to other memories discussed in this report. MOS RAM was generally found in that part of the computer memory hierarchy where flexible handling and fast transfer of data were highly desirable.

Price - The MOS memory price per bit (see Table 13) is currently among the highest encountered in the memory market. To offset this high price, MOS memories offer some of the most powerful data manipulation available today. As indicated by the table, some MOS memory systems could cost as much as 1 cent per bit, or more. Researchers predict that the price of the MOS dual-in-line memory package (DIP) should remain relatively constant - the DIPs capacity, however, is still projected to increase at regular intervals. Thus, with increasingly more bits available per package, the price per bit is expected to drop fast in the near future.

The bit price was calculated by dividing the advertised price by its total capacity in bits.

Table 13 shows various MOS memory designs that were thought to exhibit the potential to be used as peripheral memory. The range of capacity of these designs is from 1,440 to 6,144 megabits while the transfer rate appeared to be in the upper teens of megahertz per second. Access times of 0.28 to 2 microseconds were common. The price per bit ranged anywhere from 600 to 3000 millicents per bit, depending upon the particular design of overhead electronics and extra features desired..

Metal-oxide-semiconductors and other semiconductors are currently displacing magnetic core memories in the mainframe memory hierarchy of computers. These memories are made up of MOS packages, or dual-inlinepackages (DIPs) that are arranged on printed circuit cards (PCs). According to the Table, the highest bit capacity of the MOS chip (in each package) is about 16,000 bits. Each one of these bits can be 
individually, or randomly, addressed as opposed to the serial operation of CCDs and MBs. Thus these types of memories manage their data with far greater flexibility than serial memories. Generally, the drawback for using the MOS memories, as indicated in Table 13, is high price -however, these systems offer very high performance and low-power operation.

According to interviews with MOS manufacturers, large MOS peripheral mass memories have as yet not fared well. Some products that were made available in this type of mass storage are said to have been withdrawn from the market at this time because of poor sales.

10. Cores

Some comments as they relate to Table 14 are presented to provide a better understanding of core performance characteristics.

Storage capacity - Core memory capacities have grown tremendously since their first use as computer main memories in 1955. Today, new designs were found to reach capacities anywhere from 640 kilobits of main memory to over 81,920 megabits of computer peripheral memory. Magnetic core memories are random access in nature and operate similar to MOS memories .

Many core manufacturers claim that core memory capacity is more realistic than advertised solid-state memory capacity since little or no error correction is required (and therefore little data loss in the word) with core memory. Thus virtually the whole memory word (minus the parity bits) is available for data storage.

Average access time - The access time of core memory is moderately fast when compared to most peripheral mass memories like magnetic disk or tape (see Table 14). In some cases, according to the table, the core access time approaches those of some solid-state memories. The range of access times varies with particular designs. According to our searches, the larger the system the slower the access time. Small systems exhibited 250 nanoseconds access and large systems varied between 0.62 and 2.5 microseconds access times.

Transfer rates - The term transfer rate is not commonly used in core memories. To determine how fast bits are transferred, access time and cycle time are often specified as sufficient parameters. Thus, transfer rates must be calculated from these parameters while bearing in mind that word lengths and interleaving methods are other factors to consider. In order to verify results, the calculated transfer rates were checked by each manufacturer. As indicated in the table, the calculated of 3.2 to 13.3 megabits per second transfer rate is typical for large peripheral core memories.

Price - Several manufacturers are currently producing bulk, or peripheral, core memory systems. The advertised prices of these large sys- 


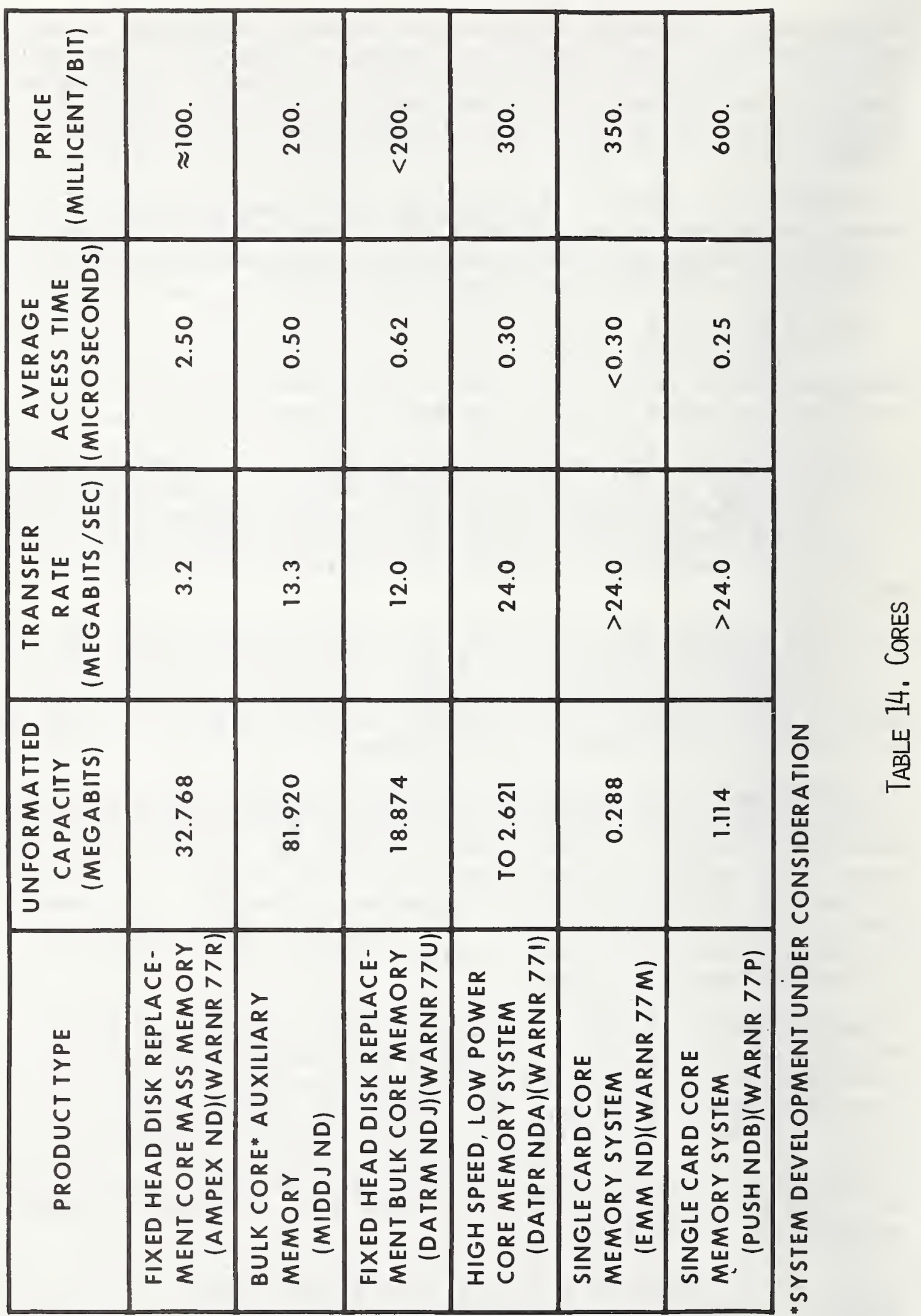


tems appear to be between 100 to 200 millicents per bit. Manufacturers of these systems claim that this price competes well with other random access mass memories, including MOS, when designed as high-storage capacity peripheral memories.

The peripheral core memory systems as shown in Table 14 range from 2.621 megabits to 81.920 megabits. These systems are constructed of many large cards so that other configurations are also possible. Typical single card capacities, not necessarily applying to peripheral memories, are shown to have a range of 0.288 to 1.114 megabits. Generally, the greater the capacity of the system, the slower the transfer rate and access time--ranges were 3.2 to 12 megahertz per second and 0.62 to 2.5 microseconds consectively for these core memories. Price is very competitive and varies between 100 to 200 millicents per bit for some large systems .

Core memory system manufacturers are exploring the computer peripheral memory market with their products. These systems are said to compete well in these applications since large core memories offer fast access times, have good data reliability, are nonvolatile, and cost less in large capacity store designs.

These designs compare favorably with fixed head magnetic disk systems in operating speeds since core memories present faster access times. One manufacturer claims a 3000 to 1 advantage.

On the other hand, this feature is costly since the price per bit is about 200 millicents for core and 2 millicents per bit for fixed disk. [AMPEX ND], [KINNP 77], [WARNR 77R]

\section{Videodisks}

The following comments about optical videodisk memories are rendered in order to provide a better understanding of the performance characteristics of Table 15 .

Storage capacity - The information stored in proposed commercial videodisks is in analog format. The information is presented by a one micrometer wide variable length pit (or oblong hole), the length of which is representative of the stored data. Up to six bits of data can be stored per pit. The disk can be divided into 32 sectors of 14,498 bits each. Since up to 40,000 spiral tracks can be stored per side on each disk, the total unformatted capacity of a commercial disk could be as high as 10 billion bits.

Average access time - The average access time of an optical videodisk is the time required to bring the "reading" laser to the right spiral track plus the necessary time for the desired data to appear under the reader optical head. In the projected systems of one trillion bits capacity, access times in the range of 50 to $1000 \mathrm{milliseconds}$ were found. In more advanced systems, multiple disk packs in "juke box" arrangements of 


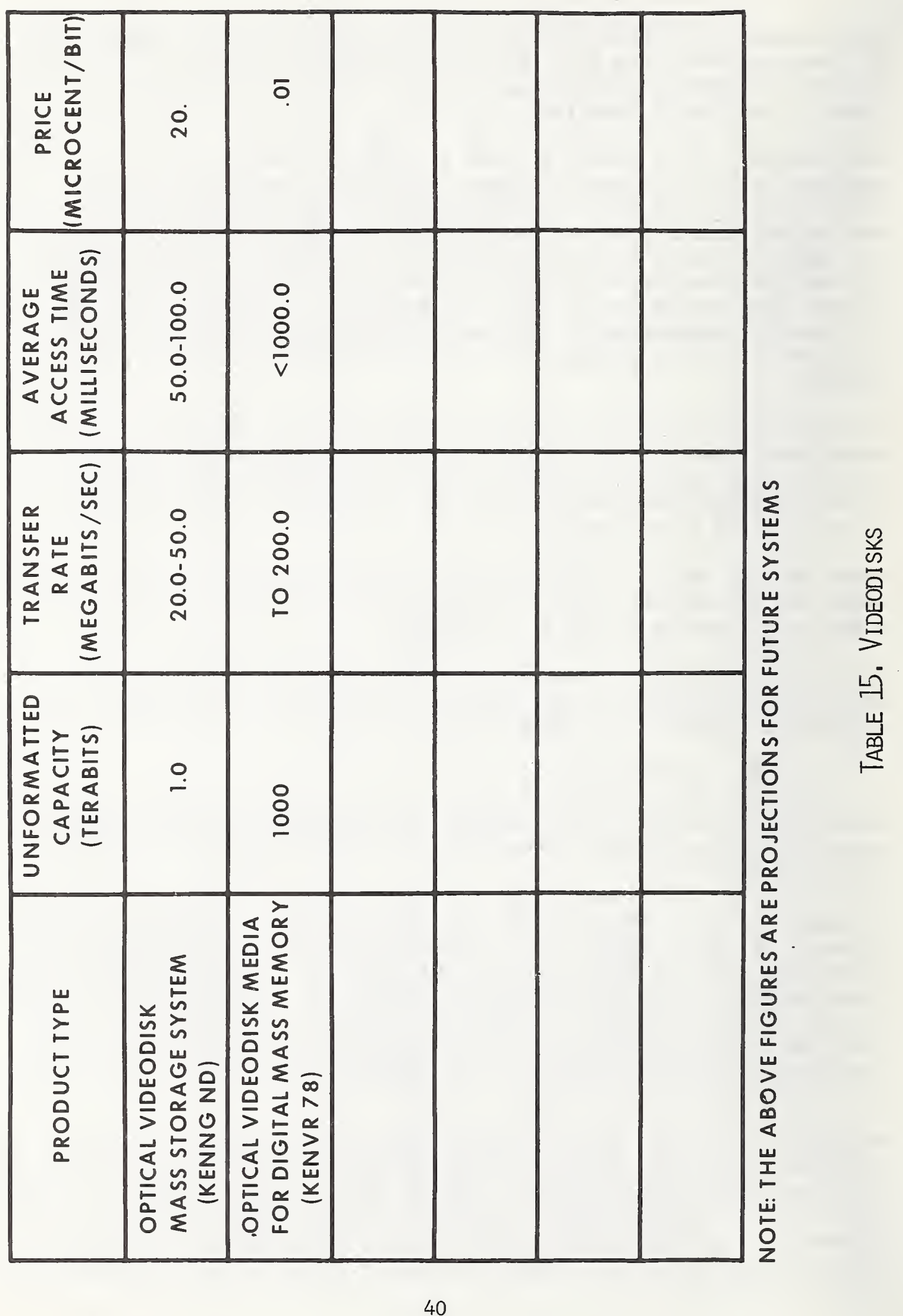


one quadrillion bits capacity (using double-sided recorded disks), the average access time is anticipated to be about 16 seconds.

Transfer rates - The transfer rate of an optical videodisk is primarily dependent on three factors:

- The rotational speed of the disk

The packing density of the data pits

- The analog-to-digital discrimination capability of the data pit analog values

Thus it follows, if the rotational speed is increased, or if more data pits are recorded on each spiral track, or if more data bits can be decoded per analog value of the recorded pits, the transfer rate is accordingly increased. Scientists estimate, if these parameters can be maximized, that future data rates of OVD mass memory systems can become as high as 50 megabits per second or more.

Price - As yet, OVD computer memory systems are still experimental and price is thus difficult to predict. Based on current costs of the few commercial optical videodisk playback systems, manufacturers estimate that a terabit read-only computer memory system could be priced at $\$ 200,000$ in the near future.

Optical videodisk memories as shown in Table 15 are still experimental in nature, but manufacturers assured us that these systems are plausible. Capacities range from one million megabits to a thousand times that value while transfer rates of 20 to 200 megabits per second are predicted. Access time of 50 to $1000 \mathrm{milliseconds}$ are not considered difficult goals to attain. At this time, the price per bit for the system is approximately 20 microcents and .01 microcent for its medium.

An optical videodisk read-only peripheral mass memory offers potential advantages, such as a low-cost and efficient storage medium and improved archival storage. The media costs, when compared to either magnetic disk or tape media of mass storage systems, are much less.

According to one research facility, the medium cost per bit of magnetic disk is 0.4 of a millicent, of tape (246 bpmm) 2.2 microcents, and of the proposed optical disk is 15 nanocents. In addition, the storage density of the optical disk is said to be 48 times better than the 246 bits per millimeter of tape. [KENNG 77]

The archival properties of OVDs are predicted to save data in excess of ten years and some estimate over 100 years. Of course, these storage techniques only allow the read-only capability, as yet.

\section{Beam addressables}

In order to provide a better understanding of the performance characteristics of beam addressable memories, some comments that apply specifically to Table 16 follow: 


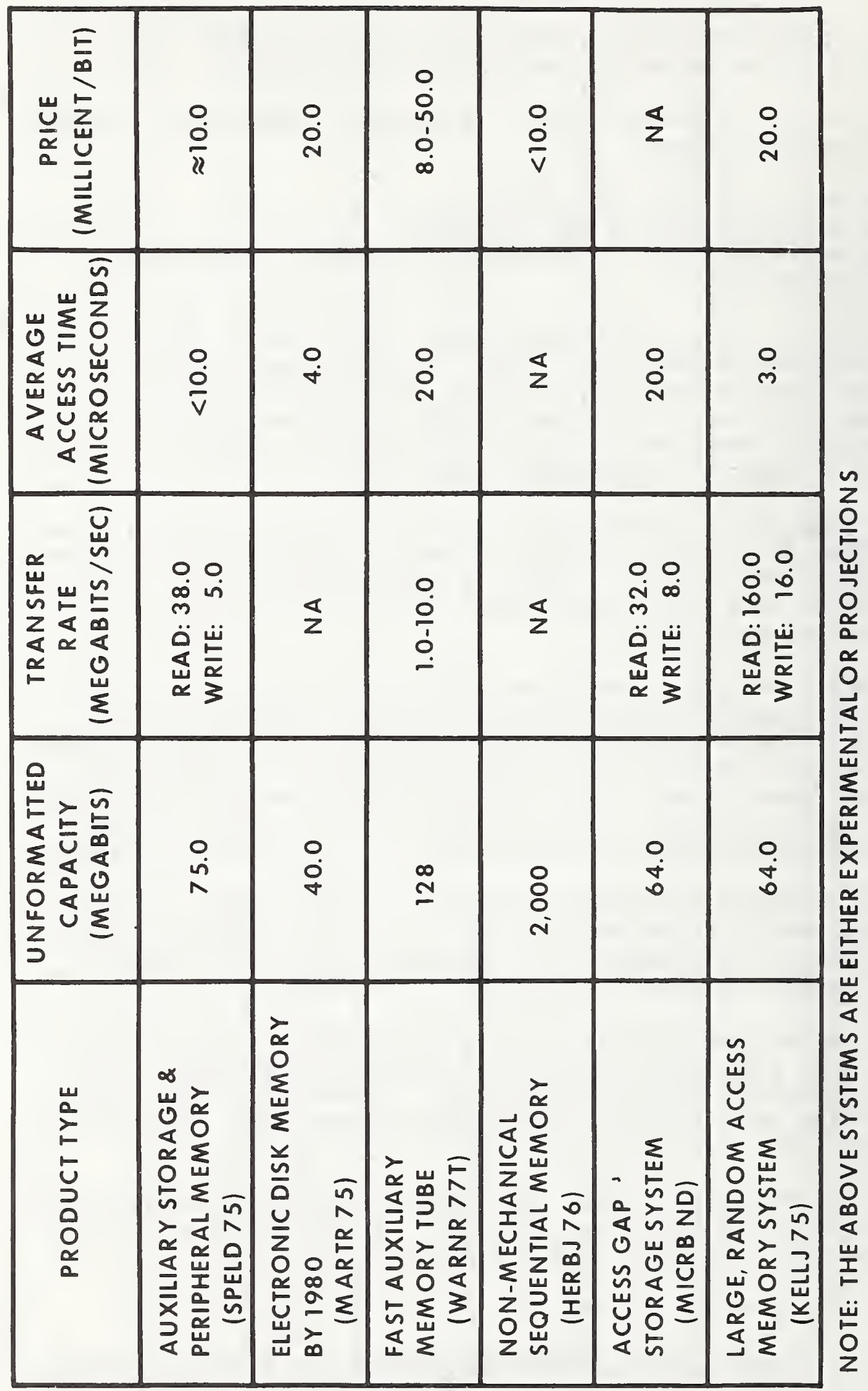


Storage capacity - The total memory capacity of a beam addressable, or electron-beam-addressable memory system is made up of several modules each of which contains a memory tube. Experimental memory tubes were found to range from one million bits to 32 million or more bits in storage capacity. Several of these modules can be connected in parallel; systems with 8,16 , and 22 modules and other configurations have been designed and are operating successfully. Generally, the parallel operation of these modules functions to form both the parallel words and the error correction storage needed to produce low error rates. [SMITD 78], [GENEL ND]

Average access time - The average access time for beam addressable memories is relatively fast when compared to magnetic disk or tape memories. On the average, the access to any data bit was specified to be in the tens of microseconds or about 1000 times faster than for magnetic disk. Data access is accomplished by addressing a block of information which is then read out serially. Because of this peculiar approach, this type of memory is sometimes called a Block-OrientedRandom-Access-Memory, or BORAM.

Transfer rate - As mentioned before, beam-addressable memories are designed with many modules in parallel. Thus, if 16 data modules are connected in parallel and each module has a transfer rate of 10 megabits, the system read transfer rate (see Table 16) would be 160 megabits.

Price - Beam addressable memories are, as yet, not commercially available. Thus pricing information is based on predictions. On the average, most research and manufacturing facilities predicted that early systems would be priced less than 1 millicent per bit in the $1980^{\prime} \mathrm{s}$. The 1 millicent figure is thought only to apply to very high capacity systems where system overhead electronics can be shared by multiple tubes. For example, a system with less than 4 million bits of storage would be priced far above the 1 millicent per bit; at this time systems with 2 billion bits are projected at prices well below 10 millicents [HERBJ 76], and a price as low as 1 millicent is not considered unreasonable.

Table 16 shows a variety of experimental electron-beam-addressable memories (in order to avoid using trade names, we refer to these memories as beam addressables). The range in system capacity is seen to be between 40 to 2,000 megabits, but these figures are not thought to be directly representative of the huge bit densities these memories are capable of. Read transfer rates of 32 to 160 megabits per second and write transfer rates of 1 and 16 megabits per second were uncovered. Access time ranges from 3 to 20 microseconds depending again on design, while price per bit showed a span of 8 to 20 millicents.

Beam addressable memories are still in the experimental stage but manufacturers project that some systems should become available in the $1980^{\prime} \mathrm{s}$. These memory systems feature high data storage capacities of up 
to 2 billion bits with fast access times of tens of microseconds. The memories are block-random accessed and have nonvolatile storage of data. No moving parts are found in these systems. Many manufacturers believe that beam addressables should be preferred over magnetic disk systems in the military environment since beam-addressable are very rugged and have the ability to withstand much shock and vibration. [GENEL ND]

III. Conclusions for Each Peripheral Memory System

A. Advantages and disadvantages of the described peripheral memories -- a comparison of price/performance characteristics of the assessed peripheral memories

1. Rotating cylinder or disk memory systems

a. Drums

Advantages

- High reliability

- Low maintenance requirements

- Relatively small in physical size

- R/W capability

- Fast access time

Disadvantages

- Small storage capacity

- Expensive (between .02 and 3.5 cents per bit, depending upon drum size, bit packing density, motor speed and the number and type of $\mathrm{R} / \mathrm{W}$ heads )

Drum units offer advantages in special situations. These advantages are high transfer rates, fast access time and ruggedness. Drums withstand severe environments in terms of physical shocks. [DATPR 77C]

$$
\begin{aligned}
& \text { b. Disks } \\
& \text { 1. Moving-head disks }
\end{aligned}
$$

Advantages

- Less costly than drums or fixed-head disks

- Comparatively large storage capacity

- R/W capability

Disadvantages

- Relatively long access time

Moving-head fixed media drives have a couple of major advantages over removable media drives. They are 30 to $40 \%$ cheaper for the same capacity, and they are more resistant to harsh environments. Since they are fairly well sealed, the disk is protected against particulate contaminants, as well as operator induced damage, such as dropping. 


\section{Stationary-head disks}

Advantages

- Fast access time

. R/W capability

Disadvantages

- Small storage capacity

- High cost

In summary, disks and drums with similar parameters compare as follows:

- Disks have the advantage in terms of capacity per unit volume

- Drums have the advantage of high speed operation and good mechanical shock resistance

- Fixed-head arrangements have the advantage of high speed operation.

- Moving-head arrangements have the advantages of lower cost and higher storage capacity

- Removeable-media units have the advantages of unlimited offline storage capacity and operational flexibility [DATPR 77C]

- Fixed-media units have the advantage of simplicity of design, requiring fewer adjustments and providing simpler servicing and greater reliability

- Rotating memories have the key advantage of flexibility for use in many applications

2. Tape storage memory systems

a. Mass Tapes

There are three types of mass storage systems: one is an automatic on-line tape library. The second is a videotape mass storage system. The third is a mass storage data cartridge system.

The following advantages can be realized for a fully automatic online tape library:

- Uses standard $1.27 \mathrm{~cm}$ tape

- Media interchangeability

- Completely automated magnetic tape storage and retrieval system

- System makes a tape library, a mass storage device, by combining the inexpensive cost of tape as a storage medium with the operating advantages of automatic on-line access

- Modular, can be expanded to 32 tape drives

- Eliminates human intervention

- Storage on tape is much less expensive than storage on disk

- Tapes are secured in a closed, protected environment until they are requested by the $\mathrm{CPU}$

- Off-line storage of 1 microcent per bit 
- Can be manually mounted

- Improved tape and file maintenance

Some of the disadvantages associated with the automatic online tape library:

- Serial access

- Mechanical in nature

- Physical size may be large

The following advantages can be realized for videotape mass storage systems :

- On-line random access

- Plug-compatible with most major computer systems

- Transverse recording technique allows the data to be recorded bit serially, as opposed to byte parallel, while retaining high channel rates

- Self-contained

- Can be used in a stand-alone environment

- Rapid random access is achieved by block address searches at a tape speed of 25.4 meters per second (mps) bidirectionally

- Eliminate conventional disk and tape libraries

- Compress off-line archives

- Reduce on-line requirements for tape and disk resources

- Improve host system throughput due to high data transfer rate

- Magnetic videotape technology is a mature technology

- Magnetic videotape achieves much higher densities

- Reduction of floor space required (10 square meters versus 195 square meters, with the equivalent bit capacity of 220 typical disk drives)

Currently, there are two mass storage data cartridge systems available and each of the systems has unique characteristics. One difference is the size of the blocks of data handled: one cartridge stores up to 50 megabytes whereas the other cartridge stores up to 8 megabytes. The data transfers of the one are oriented toward data sets of 2 megabytes or more whereas the other transfers 1 megabyte or more. Another difference is that the first required dedicated disk units to serve as staging devices whereas the other uses a common pool of disk drives, all under control of the operating system, for system staged, and unstaged operations. Still another difference is that the implementation requires user program modification for the first system but not for the second system. Another difference is that the first has two accessor mechanisms where the second accessor serves as an alternative to an inoperative first accessor or both accessors can operate simultaneously depending on the model. The other system has only one accessor mechanism. Another difference is that the first uses a helical scan and video technologies rather than the longitudinal recording which means that the first system must perform considerable more error correction [GILDJ 75]. The actual data transfer rate is higher for the second system than the first. 
The following advantages can be realized for the second mass storage data cartridge system:

- Low price/bit for on-line capacity

- Eliminates physical congestion and human errors involved with manual handling

- Faster access time than cassettes

- Automatic tape loading and unloading

- Mass storage system which utilizes tape and disk without inherent drawbacks of either medium

- Reduced floor space required

- Modular - permits field expansion by module add-on

Some disadvantages associated with the mass storage data cartridge system:

- Small capacity per cartridge

- Highly mechanical in nature

- Serial access

\section{b) Cassette}

When applying the cassette to a computer peripheral memory system, the following advantages should be realized:

- A mature technology

- Recording standard widely accepted

- Economical ( $\$ 6$ to $\$ 10)$ medium

- Package is the easiest of the three media to handle

- Can be stored and mailed because it is small and not easily damaged

- Drive mechanism is smaller than floppy disk but the same size as cartridge drive

- Cassette drive operates on a battery and requires no standby power unlike the floppy disk drive

- Cassette's plastic case provide some protection for the tape against the environment (Better protected if stored in its case)

- Minicassette is about one-third smaller and lower in cost than the standard cassette

- Minicassette requires a low-powered drive

- Higher capacity than minifloppy disk

- Nonvolatile

- Portable

Some of the disadvantages associated with cassettes are:

- Cassette's plastic case does limit performance

- The access speed for the data is limited because of the more complicated servos required for tension control and the mechanical limitations in the roller and guidance systems 
- Cassette case contains more parts than floppy disk, so the cassette tends to be less reliable

- Cassette drive requires at least two motors: one each for the take up and supply reels, and one more if a capstan drive is used

- Slow access time

\section{c) Cartridges}

The following advantages can be realized when using cartridge storage systems :

- Bidirectional

- Tape cartridge has four times the storage capacity of cassette

- Cartridge has four track (cassette has two tracks)

- Comes in more rugged case than cassette

- Cartridge housing protects the tape better than cassette

- Faster than the tape in the cassette drive due to its own mechanism within the cartridge itself

- Cartridge drive can be made more economical than a cassette drive because only one drive motor is needed to drive the elastic belt which provides the tape tension

- Increased bit density is available (252 bpmm and higher)

- Cartridge price per bit is lower than cassette and floppy because it stores more data

- Minicartridge is smaller and cheaper than the standard cartridge drive

- Lower power consumption

Some of the disadvantages associated with cartridge storage systems are:

- Cartridge medium price $(\$ 20)$ is two to three times as much as a cassette or floppy disk.

- Even though the cartridge is relatively immune to handling damage, it, like any flexible medium, should not be exposed to high temperatures or rapid temperature changes.

(After such exposure or after extended periods of storage, it is recommended that the cartridge be operated through a tensioning pass from beginning of tape (BOT) to end of tape (EOT) and return before attempting data recovery).

d) Floppy disk

The following advantages can be realized when using floppy disk:

- Data can be accessed in less than one second

- Easy to file because it is thin

- Heads on both sides of the floppy disk drive lower the access period

Some of the disadvantages associated with floppy disk are: 
- The disk drive and controller are larger, more complex and expensive than the cassette's or cartridge's drive

- Disk wear - especially in the hub area

- Varying manufacturer-to-manufacturer "compatibility" specifications

- Medium has environmental problems. It is, like tape, sensitive to temperature and humidity extremes

- Medium must be handled carefully

Magnetic tape media have been researched at NBS for an extensive period with the result that many standards and standard reference materials have been established. Furthermore, numerous articles pertaining to tape technology have been published that address tape characteristics in a definitive fashion and describe these characteristics in exact detail. Several such articles [GELLS, 74] [GELLS, 76] highlight archival data storage and the definitions of terms such as storage content, static and dynamic decay rates, lifetimes (of both medium and content), content density, cost factors, and other archival quality factors. These sources are therefore recommended as an additional guide.

\section{Optical data storage memory systems - laser beam}

The following advantages can be realized when using the laser beam mass storage unit:

- Modular unit

- Low cost (200 to 300 microcents per bit)

- Permanent medium does not degrade while stored or from repeated access

- Compact -- over 3.08 million bits/square centimeter recording density. Off-line storage of 1 trillion bits in less than 0.28 square meters of floor space

- Can be a stand-alone system or a hybrid system

- Reliable

- Longer shelf life than any other archival magnetic media

- Lower error rate

- Multiple searches and strip mount/dismount can occur simultaneously

- Reduces human intervention

- Immune to electro-magnetic radiation and computer power failures

The disadvantage associated with the laser beam mass storage system is that optical storage is in its infancy.

\section{Solid-state peripheral memory systems a. Charge-coupled devices}

When applying the charge-coupled device to a computer peripheral memory system, the following advantages should be realized: 
- Low maintenance, non-moving part solid-state peripheral memory

- Fast access times

- Low-power operation

- Block-random-access design

- High bit-storage density

Some of the disadvantages associated with CCD memories are:

- A still maturing technology

- Not yet bit-random-access

- Volatile data storage

- Poor radiation resistance

Solid-state, CCD peripheral memories offer advantages in special situations where fast access time, relatively low capacity, low-power, and low-cost operation are requirements. This situation occurs in the access gap -- or, the level in the computer memory hierarchy between main memory and mass memory. Also, these designs should offer good volumetric efficiency for small system size operation.

b. Magnetic bubble memories

The following advantages can be realized when using magnetic bubble memories:

- Nonvolatility of stored data

- Low-power operation

- Radiation resistant

- Block-random-access designs

Some of the disadvantages are:

- A still maturing technology

- Relatively large electronics overhead

- Low-speed data rates

Magnetic bubble memories offer advantages in special situations where nonvolatility, low-power and low-cost operation with block, or page, random access are requirements. Magnetic bubble memories are also very rugged and tolerate high radiation, high shock environments. Thus, at this time, bubbles are found as record storing memories in terminals and in space flight event recorders.

\section{c. MOS (metal-oxide-semiconductor) memories}

Very few MOS peripheral mass memory applications were found. Some of the advantages would be:

- Fast access times

- Low-power operation 
- Relatively good volumetric efficiency

- High data rates

The main disadvantage would be:

- High cost

5. Random-access magnetic memory systems - Core memories

When using bulk core memories as computer peripheral mass stores the following advantages should be realized:

- Nonvolatility of stored data

- Low-cost

- Exceptional data reliability

- Fast access times

- Random-access

- Matured technology

Some of the disadvantages would be:

- Relatively high operating power

- Relatively narrow operating temperature range

- New application of old technology (A reason mentioned by solidstate memory manufacturers)

\section{Alternate memory systems a. Videodisk memory systems}

Videodisk computer peripheral memory systems, as yet not available, should be able to offer the following advantages in the near future:

- Very high storage capacities

- Very low cost per bit

- Low static and dynamic wear of media

- Nonvolatility and long-term retentivity of stored data

- Portable media

- Relatively fast access times

Some of the disadvantages that might be encountered are:

- Immature technology

- Low-competition technology (as yet, very few manufacturers are involved)

- Read-only operation

- Moving parts system

Videodisk memories should excel in applications that require high volume storage of archival data. This application affords mass storage at the very low cost per bit that optical video disks (OVDs) offer. Because of the nature of the OVD medium, loss of data should be extremely rare and 
data integrity should be very good. Also, videodisk memory systems will require little floor space when compared to disk or tape memory systems. Manufacturers claim that OVDs will be the first terabit memories that are easily portable.

b. Beam addressable memory systems

When electron-beam-addressable memories are to be designed in future computer memory systems, the following advantages should be realized:

- Non-moving part memory

- Block random-access

- Fast access

- High data rates

- Nonvolatile data storage

- Low-cost per bit for large systems

- High bit-storage density

Some of the disadvantages that might be encountered are:

- Relatively large electronics overhead

- Immature technology

- Low-competition technology (as yet, few manufacturers are involved)

- High voltage system operation

Electron beam memories are still developing and should find applications to fill in the access gap area by the early 1980's. This technology, because of high data storage capability, rapid access time and data rate, paired with low-cost per bit and other factors should also strongly compete with magnetic disk memory systems.

B. Applications of the various peripheral memory systems -major and minor applications

1. Rotating cylinder or disk memory systems a. Drums

The drum is used for information of two types:

- Transient (temporary) information that changes very quickly

- Important information that must be accessible very quickly [FLORI 755]

The drum is very useful for a program that produces a large amount of intermediate results. It is also used to store a copy of the operating system. Routines in the operating system are needed quickly and used frequently. Sometimes the system cannot proceed until it receives these routines. [FORI 75]

According to one source, another use for the drum is in multiaccess computing. Here many users see the computer system as though it were a 
small console with a large amount of storage. Multiple programs are being run concurrently. When there are large programs, we may wish to bring in new copies of the program at frequent intervals, taking out the copy of the program which is presently running. The drum provides a place to keep a copy of programs. The act of interchanging these programs is called swapping. [FLORI 75]

Drums provide a page-swapping area for some systems using virtual memory. Drums can be used to store frequently used utility software and perform buffering and queueing tasks. Message switching for Telex networks and telephony are some typical examples of drum usage. Because of their ruggedness and high reliability drums are used in many military applications where these characteristics are required.

$$
\begin{aligned}
& \text { b. Disks } \\
& \text { 1. Moving-head disks }
\end{aligned}
$$

Moving-head magnetic disks are used in applications where the head positioning time can be tolerated because fast access time is not a requirement. Spooling, multiprogramming and time-sharing are some applications. On-line data entry, data communications, data base management and information retrieval are some other applications. [DATPR $77 \mathrm{C}]$

Moving-head disks are used as buffers, paging devices and file storage in computer systems. Most of the modern concepts of information handling and processing that are leading to wider application of computers stem from the presence of disk storage units. [DATPR 77C]

\section{Stationary-head disks}

Fixed-head magnetic disks are logically the same (in a computer sense) as magnetic drums. Fixed-head disks may be used for the same type of applications as drums, except in those areas where drums are used because of their ruggedness. They may be used to store a copy of an operating system or to store intermediate results in applications where a program may generate many such results. They are used in multiaccess computing applications.

Fixed-head disks are appropriate in applications where the head positioning time of moveable head disks cannot be tolerated. Some uses include process control, power distribution, numeric control and seismic applications. Sometimes they are used as buffers or paging devices in computer systems.

2. Tape storage memory systems

a) Mass tapes

According to the results of our searches, tape mass storage systems are reported to be used in many applications. Specifically these systems are designed to: 
- Replace manually-mounted magnetic tape

- Allow installations to service ever growing numbers of interactive users

- Place fast growing data bases on-line particularly in a multicomputer environment

- Eliminate human intervention

- Improve resource management control and file maintenance

- Consolidate data bases

- Reduce operational cost

The operational cost of running an installation today accounts for a third of the average computing budget [MORGW 75]. The search for savings in this area has led to the development of automatic on-line tape libraries. Some manufacturers of these systems claim that this primary application reduces the cost in the areas of space, personnel, media damage, CPU partition time, errors, reruns, delay and inefficient equipment utilization. Also, the operational complexity of scheduling jobs, media, and machines is reduced.

\section{b) Cartridges/Minicartridges}

A practical application of cartridges to computer storage requires the following:

- High-density recording capability

- A design of sufficient precision and simplicity to yield reliability and interchangeability

- A design which can be produced at low-cost

- A large enough capacity to meet most computer applications

The prime application area for cartridge drives is for unattended data logging, particularly in harsh environments where dust, dirt, heat and cold are problems. Most high volume data logging operations usually cannot permit too many errors to occur. For instance, a system that monitors a chemical plant operation could create a hazardous condition if the logger erred too often. Other applications of tape cartridge drives include:

- Replacement for paper tape punch/reader

- Numerical control of machine tools

- Small business system

- Program and data storage with microcomputer system

- Data logger for the telephone industry

- Printing industry which is beginning to use magnetic tape control of press operations

Minicartridge intended for point-of-sale terminals, automatic typewriters and similar equipment in which size is critical. 


\section{c) Cassettes/Minicassettes}

Cassettes have the following applications:

Operate in hostile environment

- Data logging

- Numerical control of machine tools

- Seismic/hydrological survey

- Airborne equipment

Terminals

- Remote batch

- Data entry

- Point of sale

- Telecommunications

Minicomputer

- Program load

- Data storage

- Master file

- Subroutine files

Calculator

- Calculator programs

- Calculator data

Business systems

- Word processors

- Automatic typewriters

- Editing typewriters

- Programmable calculators

Cassette drives, with their relatively small size and low power requirements, are highly adaptable for remote data collection. Also, they are used as archival storage systems on low cost minicomputers and for data file storage on many small business systems.

Cassettes are used as mass storage memories for microprocessor controlled data acquisition systems such as those used to monitor industrial machinery. Cassette drives have been used for many years in portable inventory equipment. The inventory count is entered by a keyboard that is hand-held or carried on a strap or belt and then made available for use on data processing equipment. Some systems are capable of being connected over telephone lines to a large central computer which collects and processes the data. The same cassette drive is used for collection and transmission. Using the cassettes for word proces- 
sing systems, two drives are required so that new data can be merged and old data can be arranged by taking the old data set from one drive, modifying and adding to it, then recording the new data on the second drive.

Minicassette applications are:

- Hand-held terminals

- Desk top calculators

- Microprocessor program loader

- Data acquisition storage

- Word processing

- Minicomputer storage

With low power, small size and light weight, minicassettes are ideal for remote and portable battery-powered applications. The drive requires less than 1 watt when operating and almost zero standby power. The data storage requirements are low, and many days' worth of information can be collected on a single minicassette.

Also, they are suitable for the personal and home computer market due to their low cost and relatively low data transfer rate.

\section{d. Floppy disks/minifloppy disks}

Floppy disks have the following applications:

- Data entry systems

- Minicomputer systems

- Terminals

- Telecommunication

- Word processing systems

Word processing systems are one of the most demanding areas of storage:

- Storage medium must be easy to store and load into its drive, since few operators are experienced with computer controlled devices

- Searching for data when editing requires rapid accessibility

Applications for floppy disks began with data entry to replace punched cards, but have grown to include uses such as disk systems on minicomputers and storage devices for word processors. Using the floppy disk for word processing systems offers two advantages: both the new and old data set can be contained on the same floppy disk, and data can be accessed faster than with cassette or cartridge -- in less than one second. A cassette/cartridge requires longer searching time -- from several seconds to more than a minute. But, by the same token, the floppy disk may be less desirable than a cartridge if more data must be stored (20 pages) than the floppy disk can handle (see Table 17 for 
comparisons). This is the tradeoff in term of capacity versus access time. Minifloppy disks have the following applications:

- Word processing

- Data entry level microprocessor systems

- Intelligent calculators

- Program storage

- Hobby computer systems

- Intelligent terminals

- Small business machines

The minifloppy disk device, with its small size and random access capability, is compatible with the fast growing word processing and programmable calculator market. The drive is one fifth the volume of the larger floppy disk drive and uses a battery drive motor, consuming $15 \mathrm{w}$ running and $7.5 \mathrm{w}$ in standby.

\section{Optical data storage memories systems--Laser beam}

The applications associated with the laser beam mass storage system as an EDP peripheral are:

- Provide mass storage for data bases and libraries

- Replace archival tape files

- Very high density data storage for archival purposes

- Program backup

- Security files

The laser beam mass storage systems can be a stand-alone or a hybrid system. It can provide both EDP mass memory storage and document image storage in the same system. [PC 77]

\section{Solid-state peripheral memory systems \\ a. Charge-coupled devices}

Charge-coupled devices are reported to be used in the following applications :

- Computer file, or swap memories

- Fixed-head disk replacements

- Microcomputer memory systems

- Intelligent terminal memories

- Fixed-head drum replacements

The above memory applications of the CCD can be classified into two categories:

- Mass, block-random-access memory

- File, or page, swapping memories 
In mass storage applications, CCD BORAMs are currently available as separate subsystems while file memories were found to be part of new computer main memory designs.

It occurs often that electronic computing systems need to be upgraded. In this process difficulties are soon encountered in rearrangement of old and new subsystems because of rigid space, power, size, interface requirements and other criteria. Manufacturers of CCD, disk replacement memories, advertise that their systems are very adaptive and should fit easily in upgraded or new systems. The small size, nonmoving, low-power characteristics of their products are their reasons for advertising a more flexible design than their mechanical counterparts.

When anticipating introduction of these new systems, software changes should be looked at before making a firm decision. Changes of this kind might be necessary since system throughput should be upgraded -- some electronic disk memories are reported to increase data transfer to the computer as much as 40 times. Thus, as part of the reconfiguration estimate, it is suggested that software costs be discussed with the memory manufacturer.

\section{b. Magnetic bubble memories}

According to the literature and interviews with MB manufacturers, the following system replacements are considered feasible depending on the application:

- Cassette replacement

- Flexible disk replacement

- Paper tape replacement

- Solid-state semiconductor memory replacement

Mechanical, moving memories, identified as the top three in the above applications are said not to perform as well as some MBs.

For example, one MB design shows three times the bit rate of a flexible (floppy) disk, uses one tenth the power, measures one tenth in size, and operates at 1000 times better error rate. This comparison applies to a bubble module with an advertised capacity of half that of a floppy disk. [ROCKW ND], [ELECT 76B]

Another application is found in a memory data terminal product line that became recently available. These types of terminals can keep track of permanent records and can be used in applications such as credit verification, real estate inquiries, wholesale and retail order entries, just to name a few. [DIGID 776], [TEXAS ND8]

A future application of magnetic bubbles is said to exist in handheld calculators. Instead of using solid-state semiconductor memories, bubble memories can be designed into handheld calculators to store much larger programs. MBs display an advantage over semiconductors in this 
application since $M B$ data storage is nonvolatile and MB storage capacity is greater per unit volume. Calculators with bubble memories thus retain their information when the power to the unit is turned off.

\section{c. MOS}

Metal-oxide-semiconductor peripheral memories are said to be still too costly for immediate use. Manufacturers who relate this information express the opinion that too few applications would require such MOS mass memories at this time; however, this could change in the near future.

\section{Random-access magnetic memory systems -- Core memories}

According to the literature and interviews with core manufacturers, the following applications were found:

- Computer peripheral memory (sometimes referred to as bulk core store)

- Swapping disk memory replacement

- Minor applications such as video storage, multiprocessor shared memory and high-capacity buffer

Computer main memory used to be dominated by core memories -- as of this date this application of core has been reduced significantly since the advent of semiconductor memories. However, core memories still enjoy long life, especially in large memories. Core memory modules are becoming larger and larger. Whereas formerly 8 kilobyte modules were popular, now 256 kilobyte single card systems are available. [DATRM NDJ]

Core modules of this size can be configured into complete systems with 2 megabytes of directly accessible storage. One manufacturer claims that his products can be configured into systems of up to 1000 megabytes. [WARNR 77V]

Systems of such high capacities coupled with data retentivity and random-access storage find applications in the mass peripheral and access gap areas of the computer memory. In the latter area, bulk core offers increased performance over swapping disks; in mass peripheral memory, 1000 megabyte bulk core systems exceed the storage capacity, access times, error rates, and system ruggedness of many multiple spindle magnetic disk systems. Transfer rates are comparable.

\section{Alternate memory system}

\section{a. Videodisk memory systems}

According to our resources and interviews with manufacturers of future computer optical disk memory systems, the following major applications were proposed: 
- Computer large mass, archival storage

- Computer controlled information storage and retrieval systems

The ultrahigh packing density, wide bandwidth, and high signal-to-noise ratio potential of future OVD memory systems, coupled with its potential for ease of handling of the platters and instant record and playback, have made this technology attractive for many information storage applications [KENVR 78] Also, the storage medium is said to display extremely good archival properties paired with excellent portability. Another advantage of using computer OVD memory over other types of mass storage is that its medium, or disks, experience little or no wear both when in use and in storage. It, thereby, differs from tape for example, which wears when in use. This OVD characteristic can be a costsaver since recorded information would not have to be rerecorded as frequently because of wear.

In applications such as archival mass storage, OVD's appear more promising than magnetic disk and mass tape systems. Of course, this comparison ignores the fact that OVD's are read-only memories and magnetic disks and tape are $\mathrm{R} / \mathrm{W}$, erasable memories.

b. Beam-addressable memory systems

According to the literature and interviews with manufacturers of future beam-addressable memory systems, the following applications were suggested:

- Fast auxiliary memory

- On-line, random-access peripheral memory

- Main memory extension (in combination with semiconductor cache)

Electron beam addressable memories should initially be cost-competitive with fast auxiliary storage designs, and eventually with most on-line random-access peripheral memories, but with superior performance over conventional memories. Equally important is their potential use as main memory extension in combination with semiconductor cache. In this application they are said to have a large price advantage at comparable performance. [SPELD 75]

In a price/performance trade-off and using the figures supplied by the system parameter tables, it was found that beam addressables look between 10 and 100 times cheaper then slow MOS solid-state memory systems (over 40 megabytes in electron beam addressable capacities).

In main memory extension, or fast auxiliary memory applications, beam addressables show about a ten times slower access time; but they can approach MOS transfer rates when many memory tubes are connected together in the design for parallel transmission of data bits. 
C. Projections for Various Peripheral Memory Technologies Current Trends in Developments and Markets

1. Rotating cylinder or disk memory systems

a. Drums

Nothing significantly new or different has occurred recently in drum technology nor does there seem to be anything on the horizon, except that bit density (per track and per device) has been increasing over the years, with decreasing cost per bit as the result. One manufacturer expects this to continue, estimating halving of the bit cost in the next two years. Interest of manufacturers and users has shifted primarily to the disk market.

Drums are becoming a smaller percentage of the total peripheral memory market, although the drum market itself is growing slightly. In many cases, fixed head disks are used instead of drums because they are logically the same, generally cost less and have greater capacity.

There is now only one major computer manufacturer that is actively marketing a wide range of drum units. These are offered only for the larger computer systems. [DATPR 77C] A couple of Original Equipment Manufacturers (OEM) provide drums on a regular and continuing basis. There is one other manufacturer that will fabricate and supply a drum upon receipt of a specific order.

Figure 1 depicts the dollar value of drums shipped by U.S, manufacturers. Only one source, a drum manufacturer, was willing or able to provide us with the data for this chart. This manufacturer felt that the drum market remained at 40 million dollars from 1975 through 1977 and that it would increase to 50 million dollars by 1980. Although losing ground to magnetic disks on a percentage basis, this manufacturer felt that the dollar volume of drum business would remain fairly steady. Other manufacturers told us that they felt the future for drums was not bright but were unable to provide specific figures.

The collected information on drums shows a great probabability that eventually drums will be almost totally replaced by other types of memories. It is therefore recommended that initiation of standards not be implemented in drums for this reason.

\section{b. Disks}

According to one source, performance through 1980 will remain essentially unchanged from today's levels, but a price reduction approximately $20 \%$ each year will be experienced. Although improved disk drive performance is possible, difficulties in implementing it could spur the use of currently experimental, serial, non-mechanical storage technologies. [FETHG 76]

Over the past few years the prices per bit of moving-head disks have decreased at 20 to $25 \%$ per year, primarily due to increased storage 


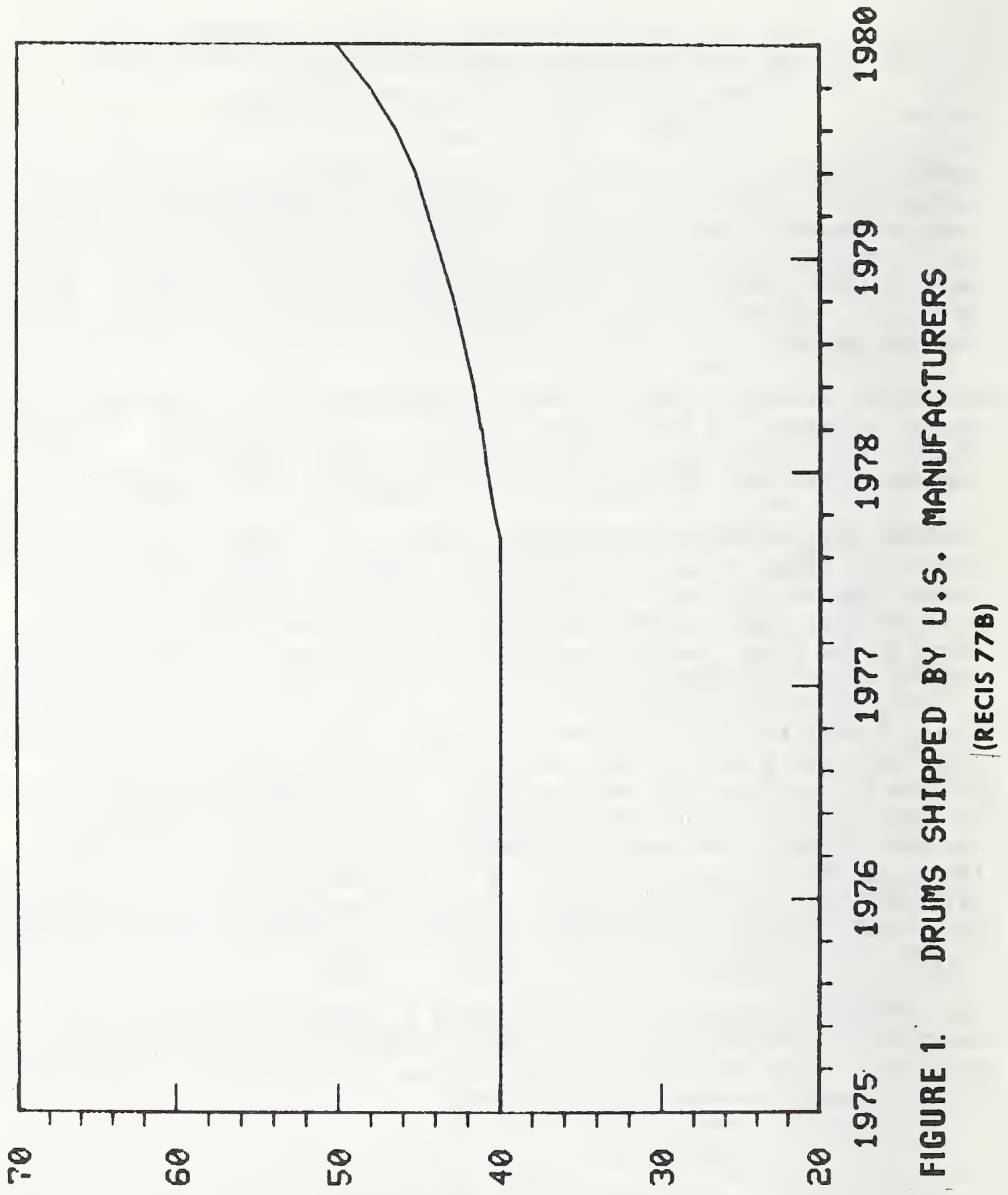

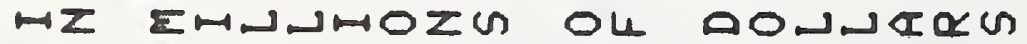

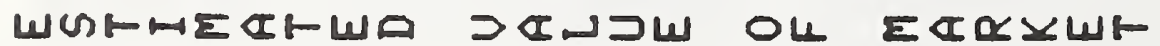


capacity per drive. At the same time, performance has improved through increases in recording density and their accompanying higher data rates. [FETHG 76]

Although some continuation in disk drive price decreases are probable in the next few years, difficulties in attaining further performance increases are predicted. An order of magnitude improvement in density, while theoretically possible, will require finer mechanical tolerances and the use of non-removable disks to achieve them. [FETHG 76]

It is safe to say that limits are being pressed in magnetic media, beyond which increased density becomes prohibitively expensive. The estimated ceiling of 394 tracks per centimeter ( 1,000 tracks per inch) is only a generation of devices away, and bit density limits of even $5905 \mathrm{bpcm}$ are not far off, being little more than double present high densities. By 1980, disk storage density will increase at most by a factor of 10, with most of the development going on in moving-head types of drives.

But even if such density improvements occur, raising a disk drive's access rate to reflect these improvements will still prove difficult. Faster access time will require the use of multiple heads for each disk surface or multiple access mechanisms for each spindle, both costly alternatives. [FETHG 76] Higher RPM is not too likely because of higher power and cooling requirements.

The trend in disks is toward non-removeable media. Primarily this is so because fixed disks offer lower cost and higher reliability.

Today's disk drives are smaller and lighter than they were previously and therefore require less space. In instances where the drives are part of a computer system in which the central processing unit (CPU) and other peripherals require a computer room environment, the size of the disk memory system becomes a significant cost factor in view of the price per square meter of such facilities. However, many of today's drives do not demand a computer room environment since the trend is to include an internal air filtration system to cope with all but the most extreme conditions of air contamination. Current disk drive engineering also results in today's disk drives being comparatively tolerant of temperature changes. [BARCM 77]

For example, today two 300 megabyte drives have the storage capacity previously achieved by six 100 megabyte drives. This improvement shows approximately a 30 percent reduction in electric power consumption.

Since there is a reduction in required pack changes, fewer operators are necessary and because of a reduction in the number of disk drives, fewer maintenance engineers are required. As a result of these improvements operation and maintenance costs have been significantly reduced. [BARCM 77 ] 
The size of the market for very high capacity devices will be dwarfed by the growth of demand for smaller, simpler devices in the business system area. The biggest growth in disk drive sales over the next few years will certainly be in the 10-50 megabyte range. This will be due to the reduction in small business system prices which will open up a whole new market area. [BARCM 77]

One of these smaller simpler devices is the disk cartridge drive. Most systems use a disk cartridge that includes a front-end loaded or top loaded, removable, single platter. The disk cartridge capacity is 1.24 $\mathrm{M}$ bytes to $5 \mathrm{M}$ bytes. Access times range from 35 to $75 \mathrm{milliseconds}$ and data transfer rates from $200 \mathrm{~K}$ to $300 \mathrm{~K}$ bytes per second. [RECIS 77D]

In addition to the removeable cartridge, many of the disk cartridge drives have one or more fixed disks with moveable heads, which provide additional capacity. It is understood that multiple fixed heads are about to become available on one or more of the fixed disks that will be incorporated in future systems. This is important for real-time time sharing systems that require a lot of program and file swapping from disk to mainframe memory. [DATPR 77B]

Magnetic $\mathrm{R} / \mathrm{W}$ heads with sensors of thin-film materials have left the laboratory and are entering the commercial world. They bring with them the promise of a new generation of computer disks with larger storage capacities at lower costs per bit and higher data transfer rates.

Thin-film technology represents a breakthrough in head design. A thinfilm head being produced by one manufacturer is a combination of vacuumdeposited sensors and traditional housings packaged into a complete recording head. It offers designers of disk drives at least five significant advantages:

- Improved frequency response because the sensors are made with laminated layers of magnetically oriented films that better couple flux between coil and gap

- Higher bit-packing density because of the improved characteristics of the head's magnetic field

- Higher track density with more cost-effective, precise track definition

- Increased reliability and longer head life because of an integrated structure, better uniformity in manufacturing processes, and new materials

- Cost advantages in volume production because of automated fabrication [ROSCT 77]

For disk drives with multiple-track fixed heads, thin-film technology permits increasing the number of tracks under each head. This achievement permits twice as much data storage as before with the same number of heads (or the same amount of storage with half as many heads). The combined improvements in bit and track densities result in three or four times greater data storage of fixed-head disks when compared to the same machines with conventional heads. [ROSCT 77] 
More and more computer users are moving to interactive applications because they give managers control of their operations. Files are kept constantly up to date, instead of being updated once a day or once a week, and decisions can be based on current information instead of dayor week-old data. With the cost of mass disk storage dropping steadily, it is becoming less difficult to justify the switch to interactive processing. [RECIS 77E]

Batch processing is a sequential processing operation. Batch processing requires only those files to be on line that apply to the applications program currently being executed. These files are on line only briefly and then are returned to off-line storage.

Every interactive application, however, requires that all related files be on-line all the time because they may be needed at any moment. This movement from batch to interactive systems does not necessarily require a more powerful computer but it does increase the need for expanded online storage by as much as an order of magnitude or more. This pressure for more capacity has caused suppliers to make higher and higher capacity disks available to users. [RECIS 77E]

Dual spindles for fixed and removeable disks have become popular because each spindle and head share the same electronics. This dual arrangement lowers system cost per spindle. [RECIS 77B]

Some companies now make controllers capable of handling a sizable number of spindles. Consequently, for a disk controller capable of handling up to eight spindles, the electronics cost per spindle is about one-eighth of the cost of a single spindle unit. Also, this type of multispindle controller permits designers to implement systems with a number of computers connected to one disk storage system. [RECIS 77B]

At least one company is planning to develop a semi-intelligent, microprocessor-based controller for its mass storage systems. It expects that the controller will perform many of the functions now supplied by the host computer. As an example, the controller could supply the file management function for converting mass memory to a virtual device. This type of controller could also provide transfers between devices without tying up the computer. [RECIS 77B]

Figure 2 shows the dollar value of disks shipped by U.S. manufacturers. Source A and source B are both professional consulting services. Source A's studies indicate a steady market growth for magnetic disks, reaching 2.85 billion dollars by 1980 and 3.77 billion dollars by 1985 . On the other hand, source B looked for more aggresive growth into 1979, peaking at 2.7 billion dollars at that time. From there source B anticipates a decline, precipitated primarily by charge-coupled devices (and possibly magnetic bubbles) making inroads into the peripheral memory market.

The wide acceptance of magnetic disks has necessitated the development of several categories of standards. Those standards that have been 


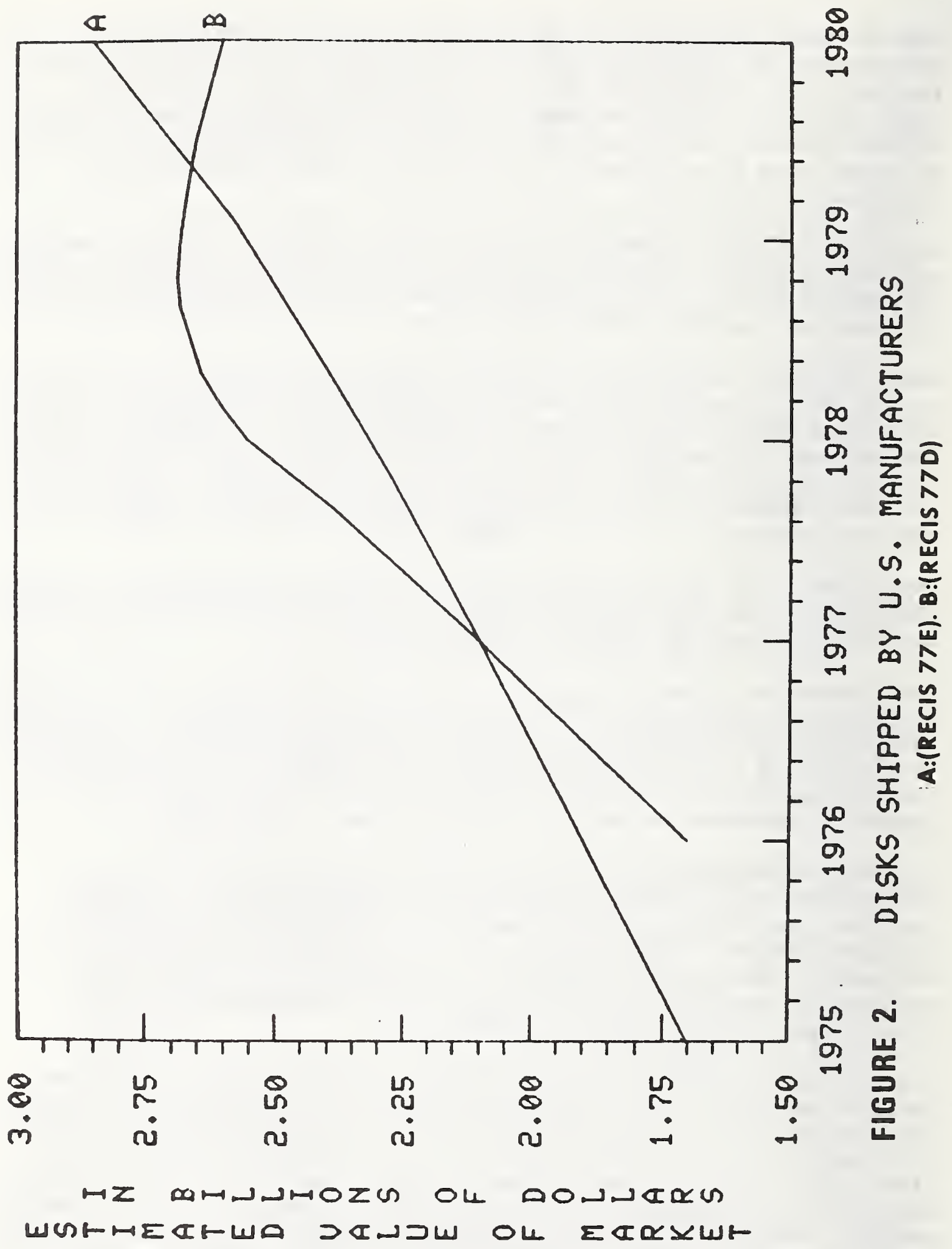


completed have proven beneficial to the user and the manufacturers. Other standards development is in progress and is expected to continue for the forseeable future.

\section{Tape storage memory systems a. Mass Tapes}

The current trends and developments in $1.27 \mathrm{~cm}$ magnetic tapes are as follows: [DIGID 77I]

- Minicomputer throughput rates increased in the last five years, and tape-drive speed and throughput have kept pace.

- Many minicomputers now provide 200 to 750 kilobytes per second throughput rates, compared with 10 to 30 kilobytes per second five years ago. But system throughput limitations still mandate tape speeds in the $63.5 \mathrm{cmps}$ (25ips) to $127 \mathrm{cmps}$ (50ips) range for the bulk of minicomputer applications.

- Common 7-track tape drives are finding less application and now represent only about $3 \%$ of the total tape drive market. The trend toward 9-track recording will make 7-track units more expensive for future users who will require them. [DIGID 77i]

- New tape drives primarily use phase encoded recording techniques although some of the most recently introduced units offer combinations of phase encoded and group coded recordings. This dual capability allows designers to achieve simplified system architecture.

- Future tape drives with 246 bpmm recording density, trading off higher price for increased throughput and the greater reliability that group code recording provides, are foreseen. All 246 bpmm units will accommodate existing $63 \mathrm{bpmm}$ formats.

- A new tape medium similar to the $6.35 \mathrm{~cm}$ data cartridge of a mass storage system could replace the $1.27 \mathrm{~cm}$ tape. The industry is moving away from $1.27 \mathrm{~cm}$ tape as a primary backup storage medium; however, $1.27 \mathrm{~cm}$ tape's demise lies far in the future.

- Future transfer rates and format of the new tape medium will be equivalent to and interchangeable with disk storage.

The number of installations of each on-line tape mass storage device tells its own story. There are over 100 mass tape cartridge storage systems with the 50 megabyte cartridge that have been installed and only 16 of the similar systems with 8 megabyte cartridge that have been installed. Also, there are over 30 automatic tape library mass storage systems ( $1.27 \mathrm{~cm}$ magnetic tape) compared to over 5 videotape mass storage systems. The era of mass storage systems (MSS) is just beginning. Much work remains to be done by MSS suppliers, operating system designers, and application system designers to utilize the full potential of MSS. The practical applications of MSS abound, and more will be discovered as the technology matures. [HOWIH 76] 
Figure 3. "U.S. OEM $1.27 \mathrm{~cm}$ tape drive market," shows the original equipment manufacturer (OEM) value rather than the end-user value. This projection shows the dramatic growth for the OEM tape drive market for two types of tape drives: tension arm and vacuum column. Overall U.S. tape drive unit shipments from 1975 projected to 1980 are 22,400 to 30,800 respectively. The major growth areas comprise miniperipheral, vacuum column drives. The advent of $246 \mathrm{bpmm}$ drives will be felt in this category. "Supermini" applications are expected to create demand in this market for tape drives for disk backup. These applications will require high transfer rates and $246 \mathrm{bpmm}$ will be a desirable feature. By 1980 , more than $50 \%$ of the vacuum column tape drives' demand will come from supermini applications. It is estimated that 10,000 to 12,000 of these tape drives will have 246 bpmm capability. [IDC 77]

Magnetic tape $(1.27 \mathrm{~cm})$, even though not suitable for random access applications, remains one of the most popular storage media. This main shortcoming is offset by other versatilities and low cost.

Magnetic tape technology quietly continues to advance with new hardware, higher density recording, and new tape packages.

The manufacturing of reel-to-reel tape drives is no longer a growth industry. Caught between the random-access capability of disk drives on one side and the handling convenience of cassettes and cartridges on the other, the tape drives seem to be just holding their market. [MINIC $76 \mathrm{~A}]$

The emergence and acceptance of high-capacity, relatively rapid, tape mass store memories are indications that tape systems have, and will have a major impact on the future computer environment. Manufacturers are confident that these systems will be in demand to meed the vastly increasing data storage requirements. Since these systems are large, they are likely to survive competition of any new technology currently in view, and since the price per lot is extremely low, introduction of standards for these types of mass tape storage devices has been beneficial and should be continued in the future.

\section{b. Cartridge}

The current trends and developments in tape cartridges are as follows:

- To improve storage capacity. The more data on each cartridge, the more competitive the drives become with each other and with other types of memory.

Most manufacturers believe that this tape medium has not reached the theoretical capacity limit. Thus, manufacturers are still working to increase bit density and the number of tracks on the tape. The results of this development for a drive with even greater storage capacity may appear in the near future. [DIGID 77G] 


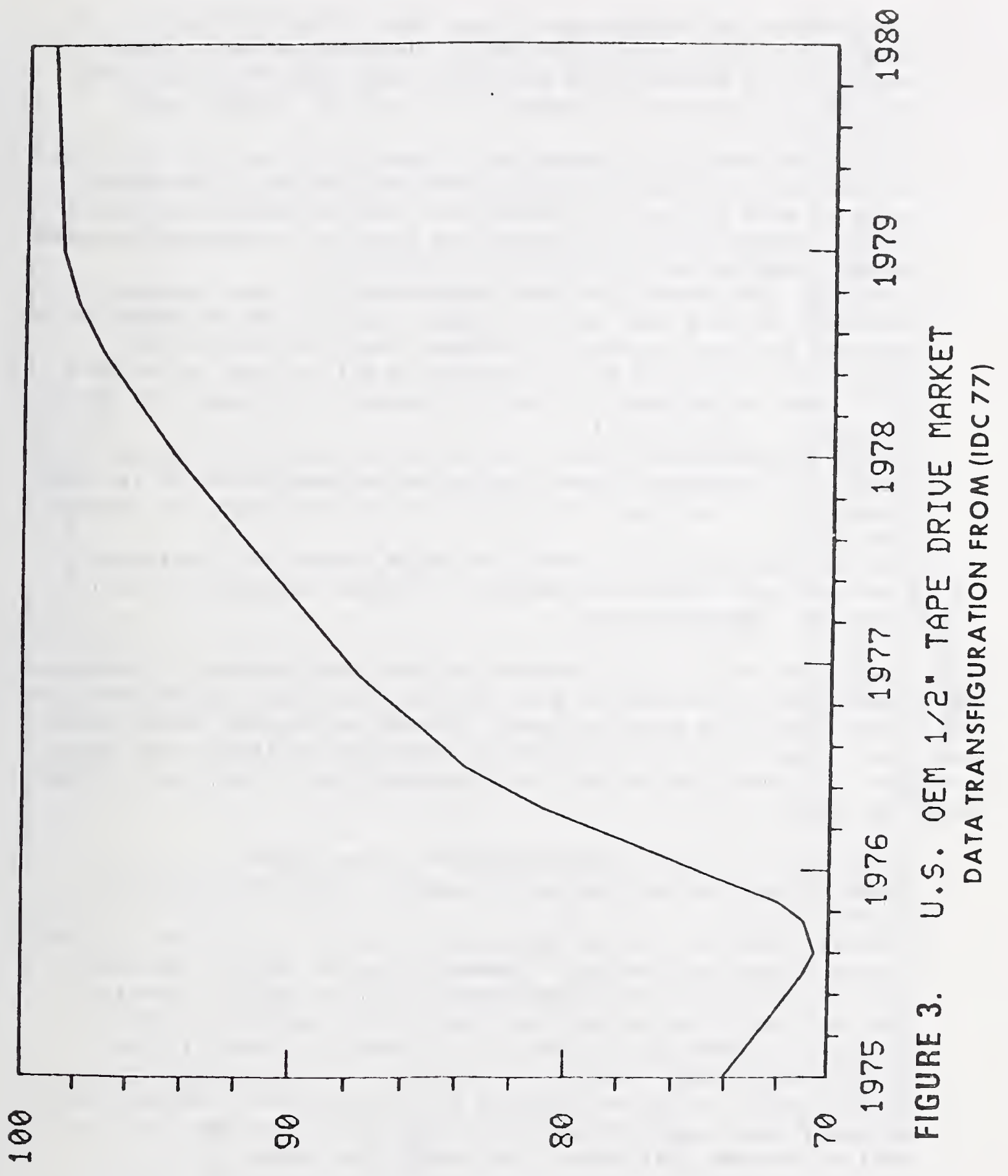

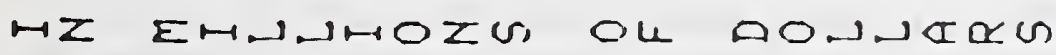

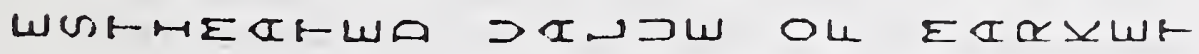


- The number of cartridge tape drives that record data at the 126 bpmm density is increasing rapidly. In round numbers, $80 \%$ of the machines sold record at $63 \mathrm{bpmm}(1600 \mathrm{bpi}$ ) and $20 \%$ at $126 \mathrm{bpmm}$. In 1978 , the rates will change to $70 \%$ and $30 \%$ respectively. [DIGID 77G]

- A 252 bpmm density is theoretically possible. The extra expense encountered in the more complex recorder/playback electronics seems to work against this potential increase because increased product volume has been lowering the price of drives and therefore making higher-priced machines less attractive.

- Cartridge limitations have kept tape speed for data transfer unchanged at $76.2 \mathrm{cmps}$ and $114.3 \mathrm{cmps}$. Speeds are not expected to increase because increased tape speed does not improve the transfer rate as much as an increase in bit density, although a $304.8 \mathrm{cmps}$ rewind speed offered by manufacturers does help system throughput. [DIGID 77G].

- Mechanism problems in cartridge drives are gradually disappearing. Currently, cartridge drives from different manufacturers are very competitive in the area of reliability, more so than they were in the past.

- Some manufacturers of low-end cartridge drives will introduce ECMA- and ANSI-compatible models to replace cassette drives in the hobby computer market.

We conclude that significant increases in the total number of tracks and flux transitions per millimeter plus various mechanical improvements are very likely within the next ten years although no drastic changes will occur soon. Standards are already being formed in cartridges and minicartridges. A continuing effort is recommended until such time as this technology peaks out.

- Minicartridge drives were introduced in early 1977.

- Manufacturers are working on increased bit density drives for minicartridges.

- Manufacturers will design and build militarized versions of their minicartridge tape drives. Commercial units are said to offer about 20,000 hours Mean-Time-Between-Failure (MTBF). Manufacturers expect that the military models will experience about 40,000 hours MTBF due to the drive's inherent simplicity and hence, reliability.

- Minicartridge drives can compete successfully with the minifloppy drives in many applications since they store more data per unit of medium, consume less power, and occupy less space.

In Figure 4, "U.S. Tape Cartridge Drive Market," shows the end-user market value projected by a professional research study. [CASWS 75B] Several manufacturers believe that it will reach its peak between 1977 and 1978 and decline to a constant level. It is expected that the floppy disk, with increased bit density and capacity, will capture some of the tape cartridge market. 


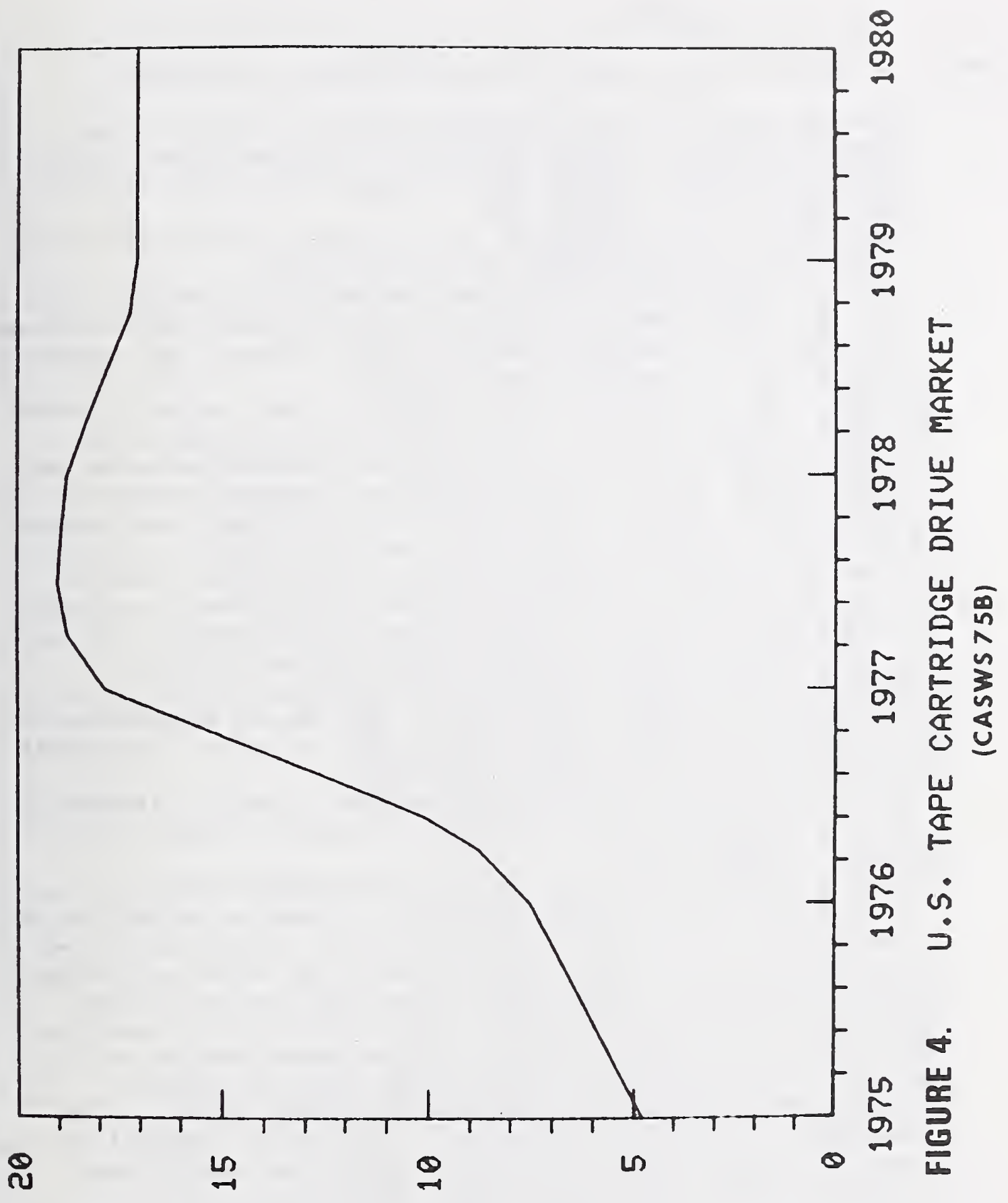

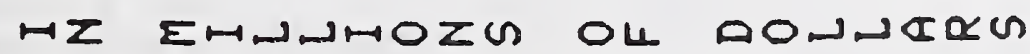

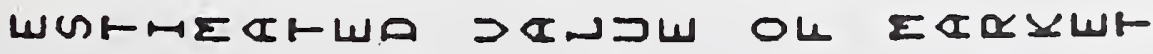




\section{c. Cassettes}

The following are current trends and developments in cassettes:

- Cassette drives are said to offer a greater reliability than floppy disk drives. Buyer confidence in product capability and quality rather than technological changes has boosted sales by about $40 \%$ in 1977. [DIGID 77G]

- Application of cassette drives in instruments and systems operating in a hostile environment will grow in 1978.

- Growing in popularity, distributed processing systems with terminals at a large number of remote locations require buffer storage and perhaps local program loading. These terminals will absorb a large number of cassette drives in 1978.

- Manufacturers will offer ceramic heads for their cassette drives in 1978. These heads will wear longer and prolong media life.

- Increased cassette drive volume will allow manufacturers to use customized LSI chips in the electronics to improve reliability and reduce costs to remain very competitive with floppy disk drives.

- Minicassette drives are accepted as useful products for instrumentation and program loading applications that require low-cost, low-power and small size storage units. This drive is used when the application does not require a faster data transfer rate or as much storage capacity as provided by full-size drives.

- Manufacturers improved the minicassette package in 1977 by replacing the pin guides with rollers. This change extended the expected number of passes across the head by an order of magnitude from about 200 to about 2000. [DIGID 77G]

Manufacturers will start offering built-in interface electronics in their minicassette drives so the customers can connect the drives to their CPUs.

- Increased cassette drive volume will allow manufacturers to use customized LSI chips in the electronics to improve reliability and reduce costs to remain very competitive with floppy disk drives.

- Minicassette drives are accepted as useful products for instrumentation and program loading applications that require low-cost, low-power and small size storage units. This drive is used when the application does not require a faster data transfer rate or as much storage capacity as provided by full-size drives. Manufacturers improved the minicassette package in 1977 by replacing the pin guides with rollers. This change extended the expected number of passes across the head by an order of magnitude from about 200 to about 2000. [DIGID 77G]

Manufacturers will start offering built-in interface electronics in their minicassette drives so the customers can connect the drives to their CPUs.

Figure 5, "U.S. Cassette Drive Market," shows the end-user market value projected by a professional research study group. [CASWS 75C] Cassette design is a mature technology which has had a head start in minicomputer applications. It is still growing in popularity. 


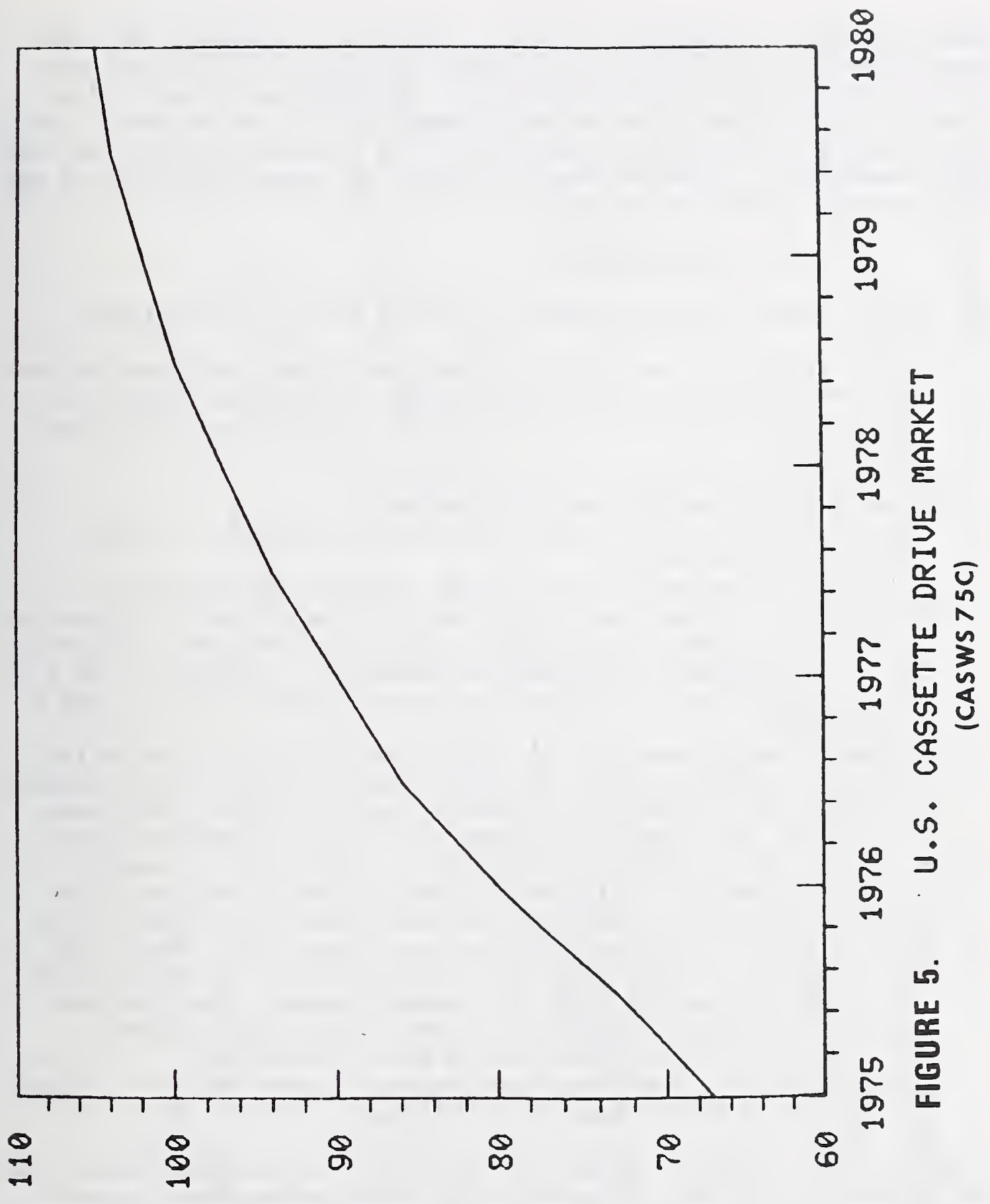

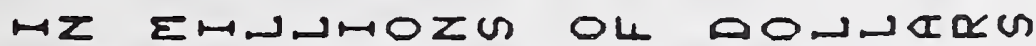

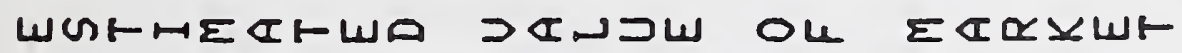


Cassettes are not expected to improve as much as cartridges within the next ten years. This technology appears to have matured and no drastic changes are expected in the near future. Standards are already being formed and will prove to be extremely beneficial for users--these standards should remain intact for reasonably long periods, while at the same time amendments of these standards will keep in reasonable pace with any major changes in this technology.

\section{d. Floppy disks}

The current trends and developments in floppy disks are as follows:

- Improved storage capacity in floppy disk drives would push prices per bit down plus the fact that competition between Japanese and U.S. manufacturers will probably force floppy prices even lower. [DIGID 77G]

- Two-sided recordings are being introduced.

- Minifloppy drives are being introduced.

- Manufacturers are increasing the use of LSI devices in their products. [DIGID 77B]

- Increased track density will become a reality in the $1980^{\prime} \mathrm{s}$. It may not first double but be incremental from 1.5 to 1.75 times its 1977 level. Some manufacturers believe that the next increase in disk track density (or tracks per centimeter -- tpcm) will be from the current 19 up to $38 \mathrm{tpcm}^{\prime} \mathrm{s}$ and possibly even up to $57 \mathrm{tpcm}$ 's. [DIGID 77G]

- Magnetic bubble memories will coexist with floppy drives in the future. Since the drives use replaceable media that can implement a library of information and bubble memories cannot, bubble memories will only displace floppies in a limited number of applications. By the $1980^{\prime}$ 's bubble memories will capture a segment of the floppy market. Only when the manufacturers have learned to produce low-cost pluggable bubble memories will these devices begin to replace floppy disk drives on a large scale. [DIGID 77G]

- Development of a large capacity minifloppy drive by replacing the usual $19 \mathrm{tpcm}$ with $39 \mathrm{tpcm}$. The drives maintain their environmental tolerances within the temperature and humidity specifications of the oxide medium on a worst case basis.

- Development of a two-sided minifloppy disk drive which will permit storage of up to one megabyte of storage.

Figure 6, "U.S. Floppy Disk Drive Market," shows several projections of the End-User market made by manufacturers and a professional research study group. Line A shows that one of the leading manufacturers projects a 70 million dollar floppy disk market by 1980. Plot B is in close agreement with Plot $\mathrm{A}$, and this prediction is made by one of the competitive manufacturers in the floppy disk market. Plot $C$ is projected by a professional research study group; they predict a 49 million dollar market by 1980. Plot D is projected by one of the small manufacturers of floppy disk their estimate is close to that of source $C$. 


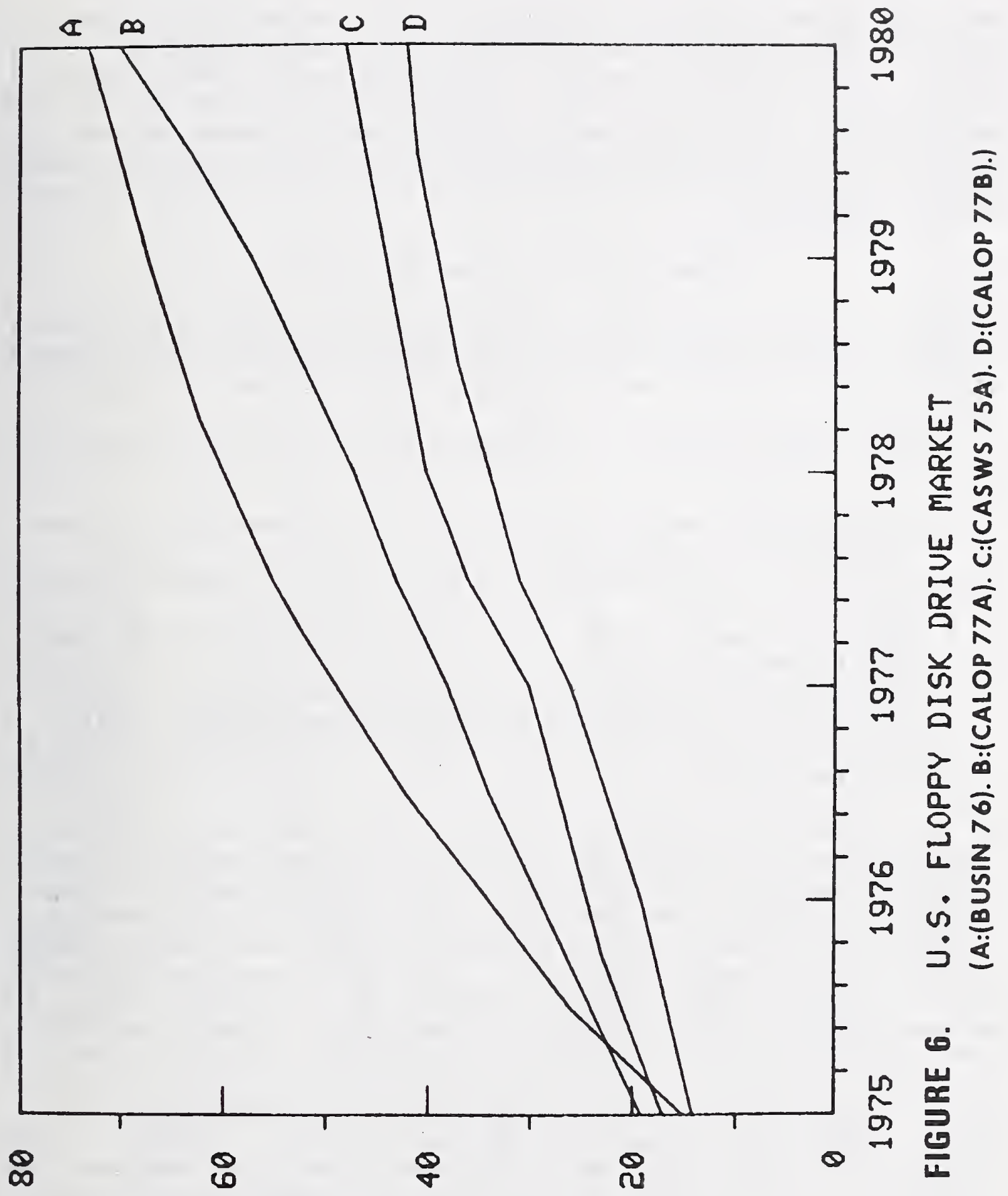

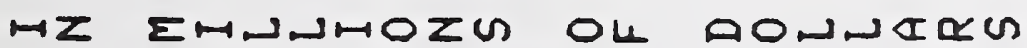

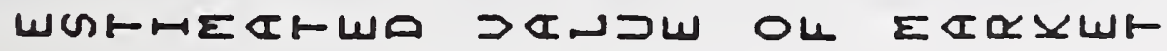


The major growth in the use of floppy disks is in data entry systems, but intelligent terminals and remote-batch applications are not far behind. A professional research firm predicts that by 1980 terminals will account for $23 \%$ of the total; $15 \%$ for data entry and $15 \%$ for point of sale systems and $13 \%$ for peripherals for small computer systems.

Fewer will be used with programmable calculators and in word processing, control, and test systems, in addition to the original use as a program loader. [FRANP 75]

\section{e. Summary graph of tape technologies}

Figure 7, "Summary graph of U.S. Tape Cartridge, Cassette, Floppy Disk and OEM 1.27 Cm Tape Drive Systems," shows the trends of the four media markets. Plot $C$ is the average of the plots $A, B, C$, and D of Figure 06 .

In summary, several trends of development of small removeable media are as follows: [SOLOL 75]

- Keypunch sales, verifier sales and rental revenues will decline throughout the decade.

- Key-to-1.27 cm tape systems will decline due to technical obsolescence.

- Key-to-disk markets will grow as they take over the large scale keyboard entry market.

- Key-to-tape cassette or tape cartridge systems will be advantageous in very low-cost data collection applications. This market will continue to expand in spite of competition from the floppy disk.

The use of small business computers has grown substantially over the last few years. In this market, cassette drives and floppy disk drives constitute the favored storage media. Cassettes have the edge on cost and storage capacity when compared to single density floppies but not when compared to double density floppies; in any case, floppies exhibit superior access times (see Table 17). Currently, cassettes find much more extensive use than floppies. Floppies can be expected to make increasing inroads, but cassettes will continue to expand in absolute numbers, though they will decline in market share, through the $1980^{\prime} \mathrm{s}$.

One of the major trends in data processing is. to capture data at the source, rather than at some later keystroking station. Because it is very costly to employ large amounts of keystroking labor, much data that might be useful often gets ignored. Take, for example, the supermarket, which takes its inventory by having stockpeople walking around the store counting cans. This inventory could be automatically controlled if the store knew which items passed the checkout. [SOLOL 75]

For this reason, it is expected that point-of-sale (POS) system markets will grow very rapidly over the next three years. Furthermore, neither cassette/cartridge drives nor floppy disk drives will play a role in POS systems where many terminals are hooked up to a minicomputer in a store- 


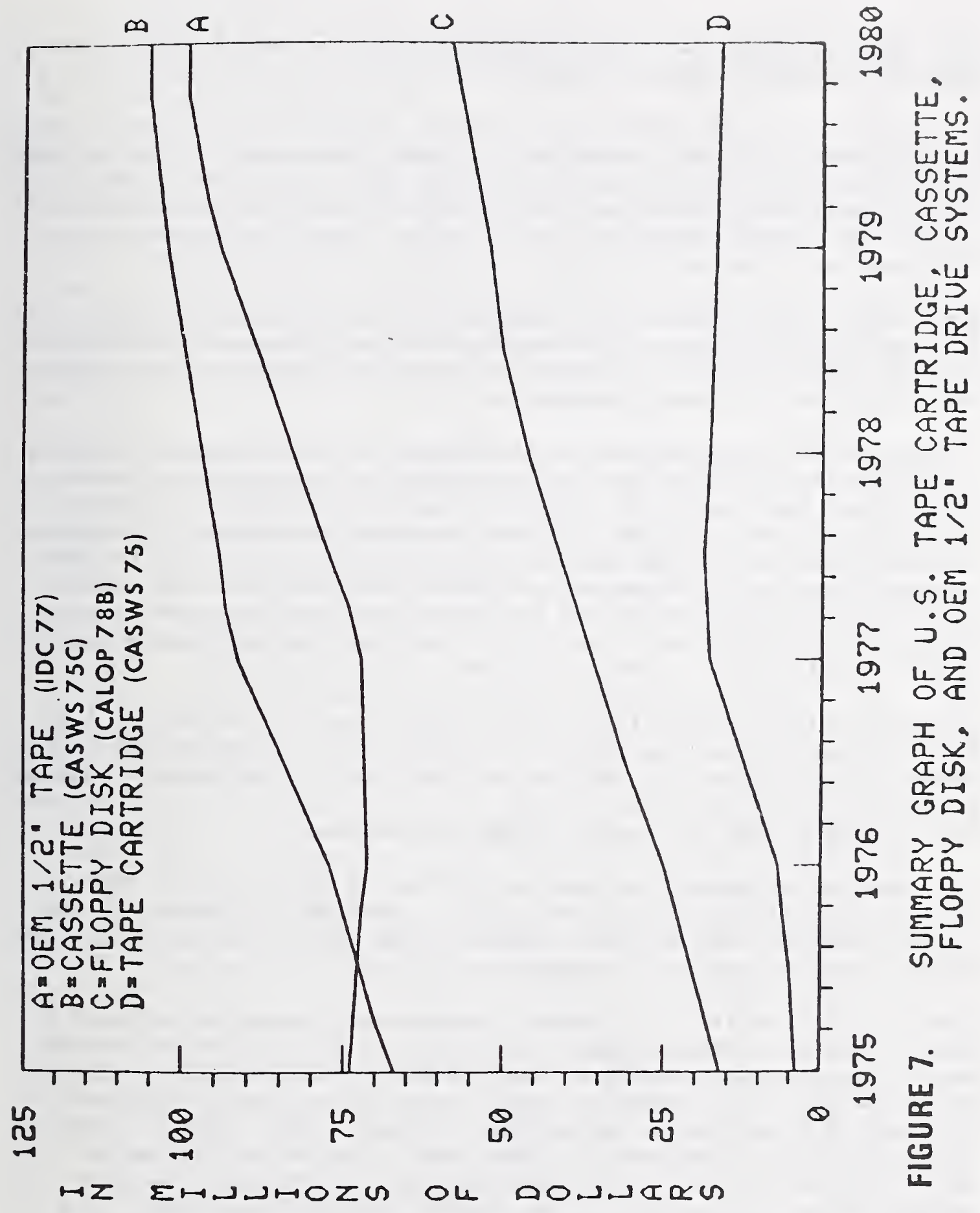

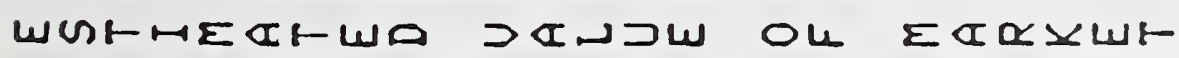


wide system. They will be used in smaller systems and in systems where each terminal operates independently.

Word processing - the composition, editing, and printing of text - uses magnetic cards, magnetic cassettes, or cartridges as its storage medium. Floppy disks will play a major role in the next generation of word processing systems because of their flexibility and storage capacity. However, the inherently higher costs of the floppy disk insures the continued use of cassette drives by independent manufacturers.

The floppy disk is the direct competitor to the cassette/cartridge. Although the floppy disks incorporate essentially the same medium as magnetic tape, they are configured as flexible disks that resemble 45 rpm records and spin continuously.

of all low-cost recording media, the floppy disks have impacted most heavily on the cassette/cartridge market in minicomputer-based products. Quick access time, about two orders of magnitude faster than a cassette's is its main feature. In data retrieval and erasure/replacement of information, the floppy disk is superior to the cassette/cartridge because it has a file structure. However, in remote data collection, this feature is unimportant, so the terminal market for cassettes is affected by floppies much less than the minicomputer-based market is. Higher transfer rate is another feature of the floppy disk. [SOCOC 75]

The relationship of the floppy disk to the large, more expensive disk drives parallels the relationship of cassettes/cartridges to the $1.27 \mathrm{~cm}$ magnetic tape drives. In the same way that certain applications dictate a cassette/ cartridge drive instead of $1.27 \mathrm{~cm}$ tape, other applications require floppy disk rather than large disk drives.

But where large computer systems use both disks and magnetic tape, minicomputer systems commonly use either cassettes or floppies because of cost factors. The medium selected depends on the user's application and the relative merits of each device. [SOLOL 75]

There is very little direct competition between cassette/cartridge and $1.27 \mathrm{~cm}$ magnetic computer tape. If the application calls for low cost, small-size, maximum convenience and low maintenance, cassette/cartridge is the answer. If it requires higher transfer rates and large storage capacity, $1.27 \mathrm{~cm}$ tape is the medium of choice. The cassette/cartridge is superior to $1.27 \mathrm{~cm}$ tape in convenience. Its medium is easy to load, small, and easy to file. The drive size is much smaller than the 1.27 $\mathrm{cm}$ tape drive. Large tape drives require continued maintenance on a regular basis whereas cassette/cartridge drives require very little care. [SOLOL 75]

Drive manufacturers will continue to improve their products by making them store more data, while operating faster and more reliably than ever; however, other nonmechanical technologies may begin displacing them. [DIGID 77G] These displacements, by CCD's MB's (and maybe videodisks in some cases) could occur in the early $1980^{\prime} \mathrm{s}$. 
One of the immediate technologies that may displace the floppy disk storage media of small systems are magnetic bubble memories. These memories could displace electromechanical recording on magnetic media in a large number of applications.

There is still a need for digital systems with a storage medium that writes and reads at very high speeds, uses little power, occupies little space - all at a very low cost per bit. An optical memory system seems to be a potential solution to the storing of large amounts of data.

We conclude that floppies are still in the evolutionary stage of development and nonetheless these systems have become very popular in a short period of time. Manufacturers are hesitant to make any long term projections of these new products, especially since this small disk technology appears to have future solid-state competitors.

Standards forming activities have been started in floppies and drafts are currently being prepared. However, it is felt that, due to continued rapid developments in this technology, standards will be formed less rapidly than those for cassettes and cartridges. But, the effort to follow up on standard setting of the floppy disk is warranted at this time since these systems offer marked improvements over cassettes and cartridges in many areas, and because of their anticipated wide use in the computer environment.

\section{Optical data storage memory system--Laser beam}

The current trends and developments of laser mass memory systems are as follows: [CALOP 78]

- New type of storage medium being developed as a slide with a measurement of $100 \mathrm{~mm} \times 100 \mathrm{~mm}$ instead of the metal film strip.

- This slide contains a metal alloy film sandwiched between two glass or plastic sheets.

- The capacity of this future medium is 10 billion bits per side. It is equivalent to 87 reels of magnetic tape with $246 \mathrm{bpmm}, 61$ meters long, and $1.27 \mathrm{~cm}$ wide. There is a possibility of a double-side capacity of 20 billion bits

- The data information hole would be cratered instead of vaporized. The size of the hole would be 1 micron instead of 3 microns.

- Future system capacity of 10 billion to 1000 trillion bits would be possible. A trillion bit storage device may occupy a volume as small as a 76.2 cubic centimeters and may sell for as low as $\$ 40,000$ in current dollars. [KACZE 77]

At this date, laser memories are still being developed and very few of the laser mass memory systems have been installed.

This technology, although still in the developmental stage, should be monitored as a possible future candidate for standards development. It would be premature to begin standards work at this time because it is 
possible that this technology will not be widely accepted by the computer community and not emerge as a viable peripheral memory device.

\section{Solid-state peripheral memory systems}

The following section provides an overview of developments and market trends in solid-state memory technology as applied to peripheral memory.

The current trends in development of solid-state peripheral memory are identified as follows:

- Increased capacity per chip, or module

- Faster data access time

- Faster transfer rate

- More bit-storage per location (multiple bit per location in CCD)

- Lower-power operation

- Lower-voltage operation (to 1 volt)

- Lower price per bit

- More intelligence, or processing power with high-capacity/high storage density chips, modules, or systems

Some of the drawbacks due to these improvements could be:

- Increased test difficulties in high-density components

This process should result in a lower bit price of system hardware paired with faster availability of desired data. These trends are likely to continue into the $1980^{\prime} \mathrm{s}$ as is described by the following.

Manufacturers related to us that the basic solid-state chip, or module characteristics, and consequently price per bit, are far from stabilized. In CCD's, the basic chip capacity is expected to jump from the current 64 kilobits to 256 kilobits in 1979, to 1 megabit in 1982, and to 4 megabits in the mid $80^{\prime} \mathrm{s}$. System transfer rates are expected to range from 5 to 10 megahertz, or higher (depending upon chip design) while the price per bit is thought to end up at a few millicents per bit by 1986. [GUIDM 78]

Existing system replacement by CCD systems is a strong trend. This means that CCD system manufacturers are aiming their new systems at "old" and established design replacement such as fixed-head disks and drums. Displacement of new disk memory systems might prove to be very difficult (according to disk memory manufacturers) since these mechanical memories are still improving their characteristics and should stave off solid-state memories like CCD's and others, as far as the late $80^{\prime} \mathrm{s}$. [PUTHS 78]

Another introductory approach is the fast-access-memory (FAM) design. This type of CCD system would fit into a memory niche, called the "Access Gap," or the level of memory hierarchy where few or no systems are currently residing. Systems that would fit in this niche should not 
necessarily exhibit high capacities but should feature fast access times and high transfer rates. [BHAND 78]

Since CCD's are fully expected to make inroads into the peripheral memory market, this emerging technology should be watched closely in anticipation of standards preparation. It is still too early to begin standards development, but after determining the course current work will take, standards making efforts should be initiated.

In MBs' current products are advertised at chip capacities of up to 92 kilobits manufacturers report that further developments will soon result in the commercial availability of 256 kilobit and 1 megabit chips.

Transfer rates of hundreds of kilobits to one megabit per second should accompany these developments.

Some sources believe that the MB commercial system is a candidate for replacing fixed-head disks and floppy disks later on. This potential market is expected to reach 40 million dollars by 1983. However, at this time MB memory technology is said to have made its best entrance in military and space electronic systems, terminals, and telephony equipment. [HUH 78] [MINIC 78A]

Magnetic bubbles will probably be taking a course of development quite similar to that of $\mathrm{CCD}^{\prime} \mathrm{s}$. As with CCD's, progress should be monitored carefully. Not enough information is available yet for standards development, but efforts should be initiated if and when magnetic bubbles establish themselves as peripheral memory devices.

MOS chip capacities are predicted to double each year through 1982--by this time the chip capacity of the dynamic type is expected to reach 256 kilobits. Also access time and power dissipation are reported to be improved accordingly. In peripheral memories, MOS will probably compete more with CCDs than with bubbles due to its inherent volatility and other similar characteristics of these semiconductor memories. MOS is expected to be priced 3 to 4 times more per bit per chip than CCD, thereby, preempting MOS from entering into the computer peripheral market except for special situations. [EARLJ 78] [BHAND 78]

Figures 8,9 , and 10 show the estimated memory market trends for CCD's, $\mathrm{MB}^{\prime} \mathrm{s}$, and MOS memory systems. All these predictions do not necessarily directly apply to computer peripheral memories but are related to, or display the potential to evolve into peripheral memories.

In Figure 8, curves $\mathrm{A}, \mathrm{F}$, and $\mathrm{G}$ were constructed from informatioli generated by "loose leaf information service" organizations. In other curves, B,C,D, and E were constructed from information obtained from interviews with manufacturers.

Most manufacturers felt that curves A and G (Source G predicts virtually no market share by 1983) were not accurate and that the source for curve $F$ was too pessimestic. Information on curves $B, C, D$, and $E$ were in- 


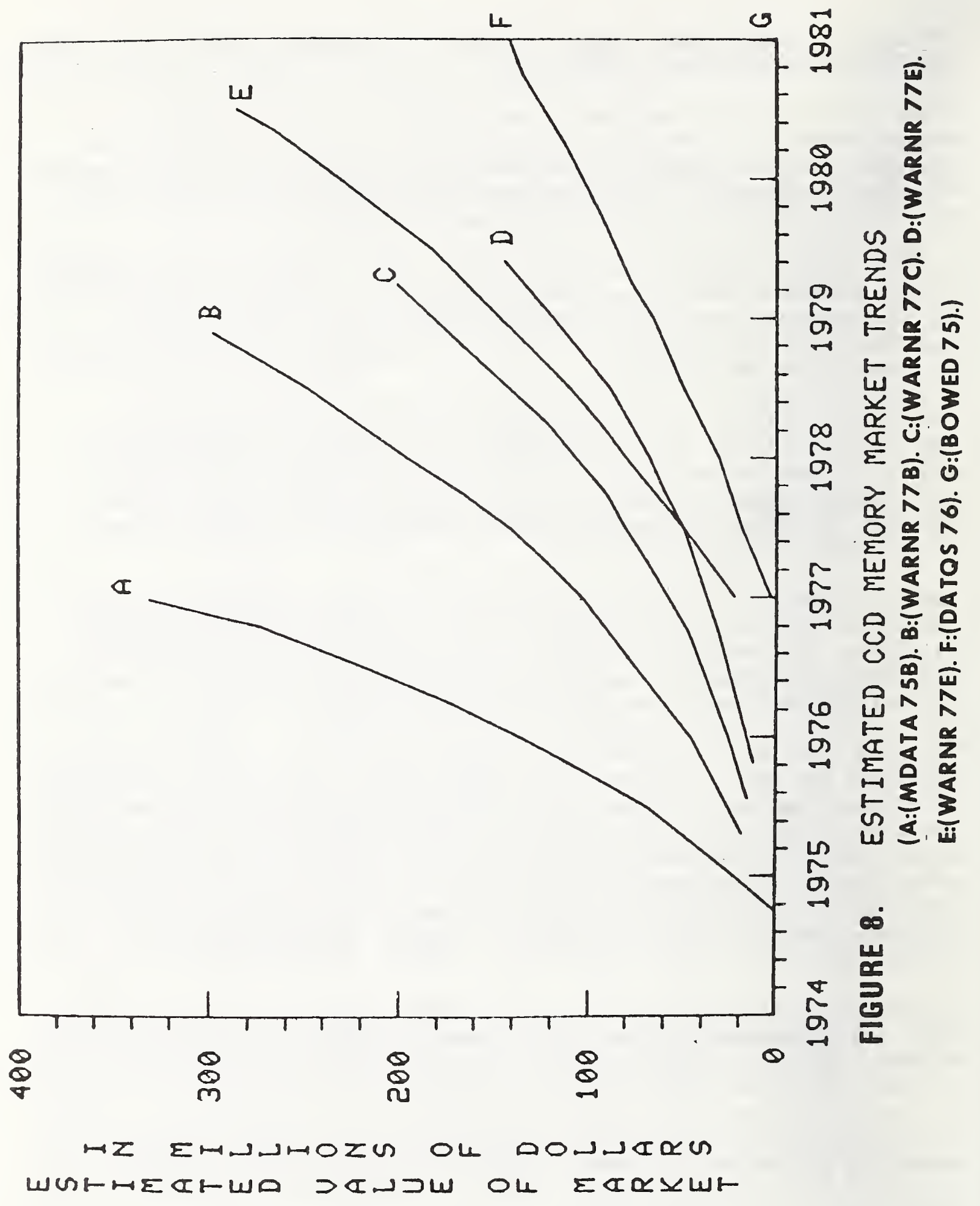


dependently entered by the various manufacturers. Of these manufacturers, sources C, D, and E are said to form the largest part of semiconductor manufacturers in the U.S. These sources felt that the CCD memory market would run between 100 and 200 million dollars in 1979 as shown in the Figure.

All sources except one $(G)$, felt that CCD's would do well in the early $80^{\prime}$ sduring this time, sharply increasing market opportunities would occur.

Estimated magnetic bubble memory market trends are suggested in Figure 9 .

Points $D$ and $F$ are predictions made by two independent MB research facilities. Neither of these provided enough information for plotting complete curves. Both sources predicted about a 200 million dollar market in either 1979 or 1980 as shown in the Figure. Both estimates appear rather optimistic when compared with the rest of the curves.

Sources of curves A and B are major bubble research and development facilities -- together they make up the largest part of MB manufacturers. Source B predicts about a 100 million dollar market in 1981 while source A feels that about a 200 million dollar market is more accurate.

Sources E and C are "loose leaf information service" organizations. Source E predicts that the market value of bubbles is somewhere between the estimates of the two major manufacturers, or about 150 million dollars by 1981. On the other hand, source C sees that magnetic bubble memories will have virtually no market share by 1983 .

Figure 10 shows the estimated MOS memory market trends through about 1980. Information for curves A and B were extracted from data gathered by industry information specialists. Figures used to form plot A are manufacturers' estimates while plot B is generated by data from an independent information source. Sources for plots D and C are independent MOS manufacturers that were interviewed.

As in the previous graphs, the dotted portions of the curves are thought to be the best continuations. These continuations are justified partly by agreement with manufacturers or follow the general slope of the existing plot.

With these assumptions in mind, it follows that sources $B$ and $C$ are approximately in agreement. Both show about a 300 million dollar market for MOS in 1977. This figure is thought to double by 1979.

Sources A and D agree on a 600 million dollar market in mid 77 . At this time, source $D$ felt that the MOS market could level off due to the entrance of other, more advanced integrated circuit technologies. No specific entrant was determined. 


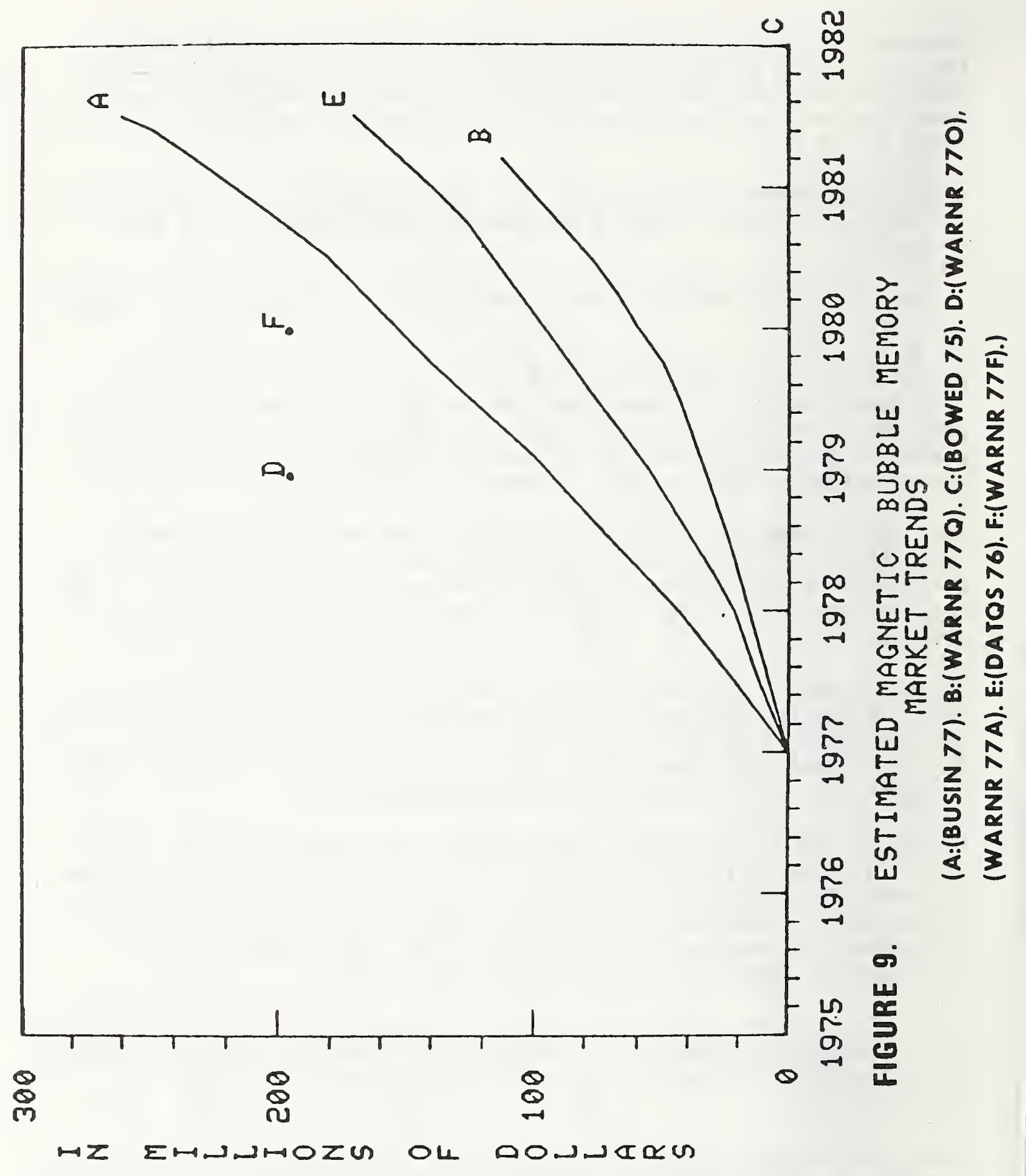

யのเ 


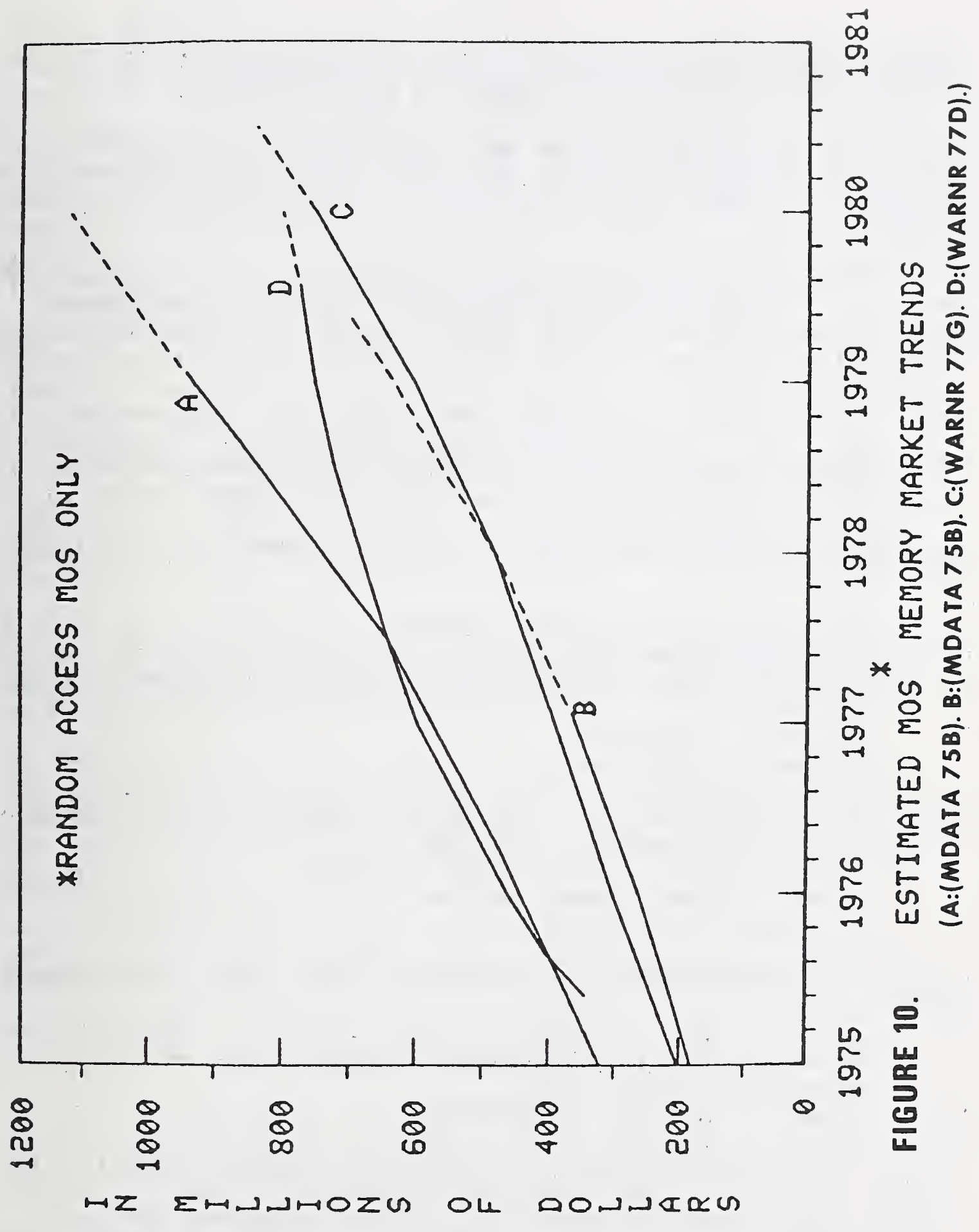

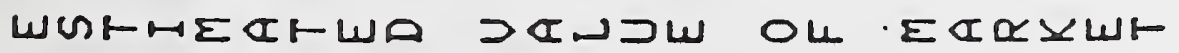


Source $A$ is the most optimistic. This information, transferred by MOS manufacturers to an independent "loose leaf information service" organization, shows a billion dollar market in MOS by mid 1979 .

Most of the MOS market was found not to be directed to the computer peripheral memory market. Thus, the curves of Figure 76 apply mostly to other computer memory applications such as mainframe memory or buffer storage.

It is doubtful whether it will become necessary to develop standards for MOS because little of the MOS market appears targeted for peripheral memory. However, this technology should continue to be monitored in case this condition changes.

\section{Random-access magnetic memory systems -- core memories}

The following section provides an overview of development and market trends in core memory technology as applied to peripheral memory.

The current trends in development of large, core memory are identified as follows:

- Increased capacity of card or module

- Faster data access time

- More core elements per unit area in the core stack (smaller core elements)

- Lower power operation

- More use of LSI circuitry

- Lower price per bit

- More intelligence, or processing with high capacity/high storage density cards, modules or systems

- A reduced number of power supply voltages

- A change from main memory application to other applications such as peripheral mass memory

Some of the drawbacks that could influence these trends in improvements are:

- Increasing fabrication difficulties of denser core stacks

- Increasing software prices

- Complex IC integration with core

One of the most important and successful memory devices is the ferrite core. Magnetic thin films, plated wire, rod memories, waffle-iron memories: all of these technologies, at one time or another, were predicted to replace and displace core as the computers' most dominant main memory element. None of these technologies appeared to have a significant effect on core; however, this trend changed when the integrated circuit appeared on the market. [KRUSG 75]

Thus, competition between IC and core manufacturers is resulting in the pursuing and searching of similar design goals as can be seen when 
comparing their listings of trends. As density, capacity, speed, and complexity increases, both technologies will be required to resort to a substantial amount of on-site processing in order to electronically respond quickly to the computer's commands and demands. This translates into more software per bit in the near future for high capacity peripheral memory whether it be core, solid-state, or other -- a factor that should be cleared up when considering these types of memories and that should be discussed with manufacturers. Currently, most manufacturers claim that their core mass memory products software is transparent when, for example, core is installed instead of fixed head disk.

Core memory cards, or modules, formerly rated in 8 kilowords with 8 bits per word (or 8 kilobyte modules) have constantly been growing. Today, complete core memory boards of 131 kilobytes or 256 kilobyte modules, and other designs, are commercially available. Progress is anticipated to continue in this technology and most manufacturers of core feel that it will never really disappear from the market although its growth rate is said to be reduced. At this time at least three major core manufacturers are producing peripheral or bulk core memories. They all expect to do well even though some IC manufacturers are expressing their doubts.

Trends indicate that the price per bit for core is losing out when core systems are small, or below 32 kilowords with 18 bits per word; but, above this size and especially in the hundreds of kilobyte word range, we found that core is still priced lower.

Figure 11 shows the estimated market trends for core. All these predictions, or curves, do not only apply to peripheral core memory but are representative of the total core market. Specific figures for constructing curves on just peripheral memories for core were found not to be available as yet.

Curves A [KRUSG 75] and C are predictions from semiconducter memory manufacturers -- the figures for these curves were extracted from information obtained from open literature or private interviews. Sources for curves B and D produce core or core/MOS memories and agree much with sources $A$ and $C$. Source $E$ is an independent research source that also agrees with the general thought of the previous sources.

All these sources, A, B, C, D, and E, claim that the core memory market should drop below the 100 million dollar mark by 1982 -- or less than 10 percent of the estimated solid-state (MOS) memory market.

Sources $F \& G$ are major core memory manufacturers who felt that the above views held by these other sources are entirely pessimistic -these sources $F \& G$ claim that their shipments, and interest in their new designs by their customers, should result in at least double that market, or 200 million dollars or more by 1982. Source F felt that the 1982 market could be as high as 250 million dollars a year. Both these sources and others are investing large amounts of resources in these new 


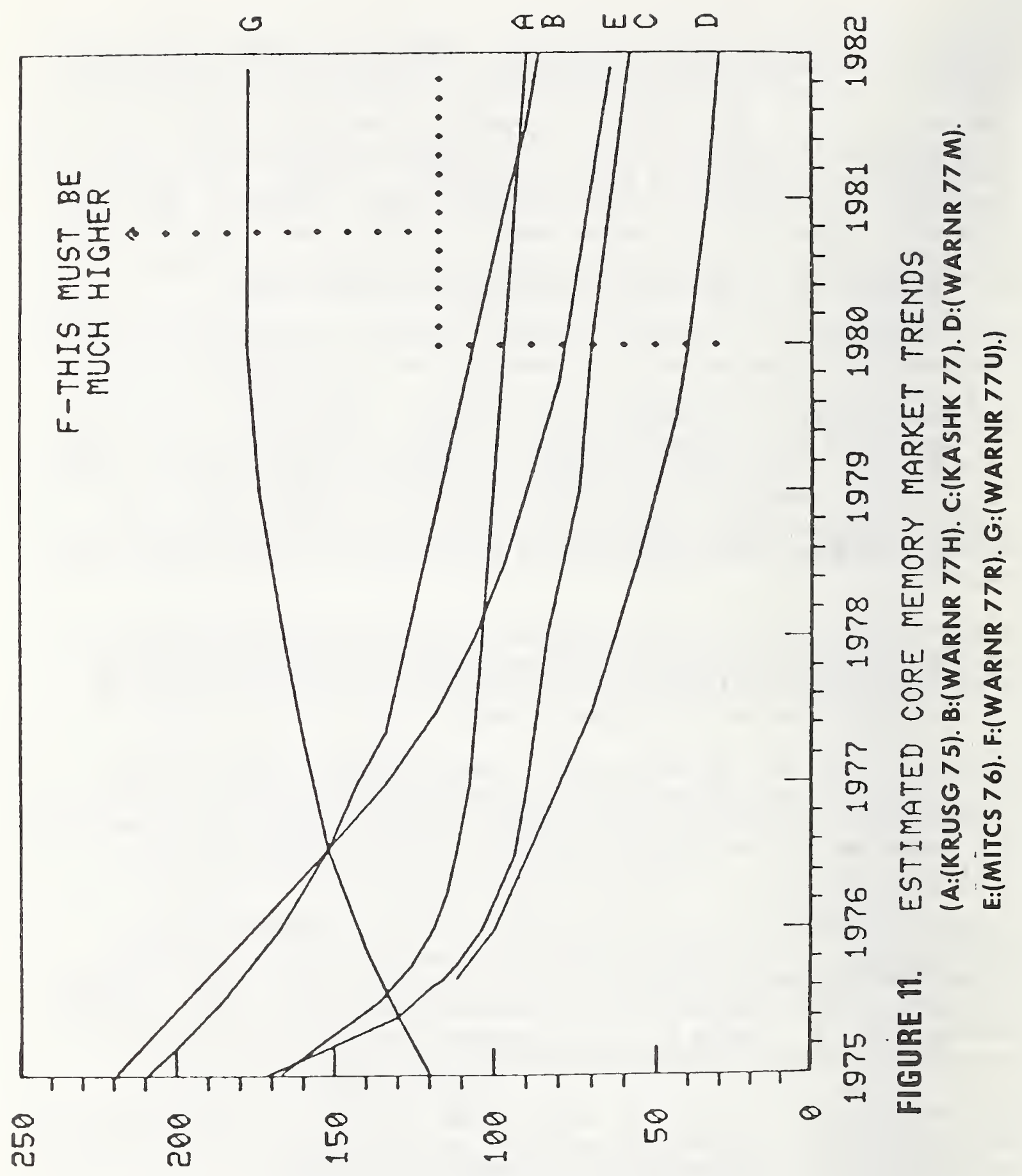

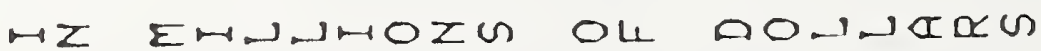

WUคHEव 
products. Production assembly lines will be more and more computer controlled, thereby perfecting fabrication to an even finer degree. Such efforts can only point to the fact that many core manufacturers are confident that they can lower their prices even more and still be successful in competing with IC memories.

Although core memories are a mature technology, specific figures regarding the number of installed peripheral core memories are not yet available. Current indications are that there probably will not be a requirement for developing standards for peripheral core memories.

\section{Alternate memory systems \\ a. Videodisk memory systems}

This section provides an overview of development trends in optical videodisk memory technology as it applies to future peripheral memory.

The current trends in development of optical videodisk peripheral memory can be identified as follows: [KENVR 78], [KENG 77]

- Ultra-high storage capacities (to a quadrillion bits)

- Decreased block-random-access times (faster laser beam switching)

- Faster transfer rates (wider bandwidth operation)

- Extremely low-wear storage media (both when in use and when not in use)

- Extremely long-term, archival storage (from 10 to 100 years)

- Increased ease of portability (to one trillion bits of portable information)

- Lower price per bit

- Lower power dissipation per bit (in the ROM mode)

- More intelligence, or processing with higher capacity systems (such as microprocessor controlled functions)

- A strong push towards error-free memory operation

- Increasingly better signal-to-noise ratios

- Increasingly smaller (in physical size) memory systems

Some of the hurdles that might be encountered in this developmental process could be:

- Slow market entry due to:

1. An apparent low number of involved manufacturers

2. A still maturing technolooy

3. Rotating media competition

Optical videodisk memory systems are not yet available. Most of the above information, thus, refers to projections that are made by the few manufacturers that are involved. However, commercial optical videodisk playback systems are available and certain facts can be derived from these systems since they bear similarity to the projected memory systems. 
Optical videodisk players have been demonstrated to both commercial and government interested parties. Program reproduction was what the producer said it would be -- better than the average television picture. One disk can be played one half-hour on each side. The amount of material on each disk is calculated to be 18.6 billion bits unformatted and 10.5 billion bits formatted. Added electronics are said to keep the error rate to less than 1 bit per billion. Each picture frame has its own spiral track on the disk and each track has its own identification number and can thereby be addressed. These features and others plus demonstrations of this commercial system at various locations provide high confidence that optical videodisk memories are very likely to soon come into being.

Small samples of the commercial videodisk playback units are scheduled for release in the midwest by the end of this year (1978). This is the market strategy by one manufacturer. No information was made available when the videodisk memory would be released -- but it is thought that much of the timetable on producing this type of memory will depend on the acceptance of the commercial player and associated videodisk sales.

Because this technology is still in the developmental stage, it would be premature to begin standards work at this time. This technology should continue to be monitored in order to keep abreast of any significant progress.

b. Beam addressable memory systems

This section provides an overview of development trends in electron beam addressable memory technology as it applies to future peripheral memory.

The current trends in development of electron beam addressable peripheral memory can be identified as follows: [KELLJ 75], [SMITD 78], [POSSG ND]

- Improved random-access times (to better than 10 microseconds)

- Improved transfer rates

- Lower cost per bit

- Higher capacity storage tubes (to 10 or 100 billion data spots)

- Increasing memory tube lifetime (to 16 years)

- Higher system capacity

- Reduction and simplification of overhead electronics (deflection amplifiers, power supplies, etc.)

- Improved long-term retentivity of stored data (improved data protection methods)

Some of the obstacles that could be encountered in the developmental process could be:

- Slow market entry due to the following:

1. Apparent low number of involved manufacturers

2. Still maturing technology 
- Refresh and calibration routines of this memory system (similar to preventive maintenance)

Prototype electron beam addressable memories have been operational with computer systems in laboratory environments. These experiments have been successful and several major computer manufacturers are currently investing in these memories. The actual areas of application have not been established but research and development trends indicate that the following is feasible: [SPELD 75]

Computer peripheral mass memory

Fast auxiliary memory (access gap memory)

Main memory extension

Most computer manufacturers that were interviewed did not reveal or were not exactly sure where the electron beam memory was going to fit in. However, a small number of manufacturers feel that this memory is a natural in the fast auxiliary memory (FAM) application.

The low-cost, beam addressable FAM would operate as a nonvolatile interstaging memory with high capacity/high transfer rate characteristics. This area of application is currently pretty much a void, -- and it seems likely that, judging by the activities of large computer manufacturers, this is the entrance portal for electron beam addressables. [WARNR 77]

The main question still to be answered is -- when?

Electron beam memories are large when compared to solid-state memories and not yet accepted when compared to disk memories. Solid-state manufacturers can "sense" the market acceptance of their parts, like MOS, $C C D$, and $M B$, much easier since these parts are easy to handle, and operate, and can be applied to numerous applications, either small or big. Beam addressables do not enjoy this luxury. The major commitment of one, or more, large computer manufacturers will determine the acceptance of this technology [THEID 78] Thus, it could be that the entrance of the beam addressable is a surprise announcement that could occur within the next five years.

Progress of the technology should continue to be monitored because this technology is still in the developmental stage. Although it would be premature to begin work on standards at this time, continued progress may dictate development of standards.

IV. Summary and Overall Conclusions

A. A summary table of the various peripheral memory technologies

The following computer peripheral memory system comparison (Table 17) provides a quick overview of how each technology compares with others in terms of technical parameters, physical arrangements and price. All values are typical. 


\begin{tabular}{|c|c|c|c|c|c|c|c|c|c|}
\hline 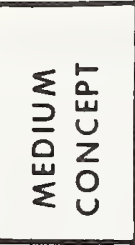 & 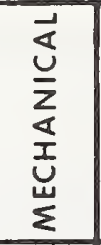 & 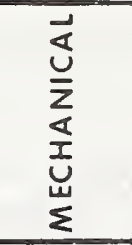 & $\begin{array}{l}\stackrel{w}{\underline{w}} \\
\stackrel{\alpha}{\tilde{n}} \\
\frac{\dddot{n}}{0}\end{array}$ & 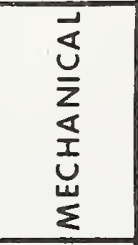 & 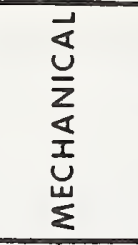 & 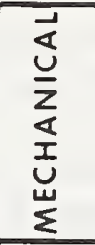 & 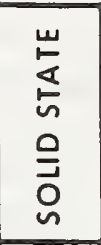 & 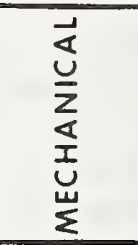 & 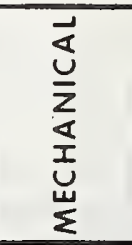 \\
\hline 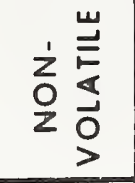 & $\stackrel{\stackrel{\Perp}{\nu}}{\nu}$ & $\stackrel{\mathscr{\sim}}{\nu}$ & $\underset{\nu}{\stackrel{\leftrightarrow}{*}}$ & $\underset{\sim}{\sim}$ & $\underset{\nu}{\stackrel{\Perp}{\nu}}$ & 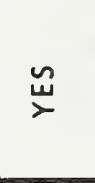 & 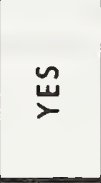 & $\underset{\nu}{\stackrel{\sim}{\sim}}$ & $\underset{\check{\nu}}{\stackrel{\sim}{\sim}}$ \\
\hline $\begin{array}{l}\sum_{0} \underset{n}{0} \\
0 \\
\sum \\
\substack{\alpha \\
\alpha} \\
\alpha\end{array}$ & 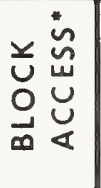 & $\frac{0}{z}$ & $\underset{\nu}{\breve{\nu}}$ & 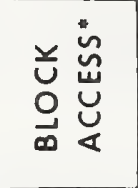 & 苍芯 & 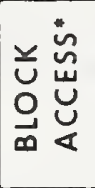 & 总芯 & $\frac{0}{z}$ & $\frac{O}{z}$ \\
\hline 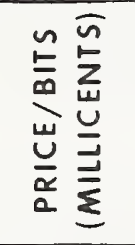 & $\stackrel{\sim}{\nabla}$ & $\begin{array}{l}\stackrel{n}{N} \\
\text { ơ } \\
\stackrel{0}{0}\end{array}$ & 오 & $\begin{array}{l}\hat{a} \\
0\end{array}$ & $\stackrel{\infty}{\longrightarrow}$ & $\cong$ & $\begin{array}{l}0 \\
\infty \\
0 \\
1\end{array}$ & $\stackrel{m}{2}$ & $\stackrel{\circ}{\circ}$ \\
\hline 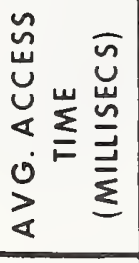 & $\stackrel{n}{\infty}$ & $\begin{array}{l}\circ \\
\circ \\
\circ \\
\pm \\
\end{array}$ & $\begin{array}{l}\text { Na } \\
\circ \\
\circ \\
\circ \\
0\end{array}$ & ले & $\stackrel{\infty}{m}$ & $\begin{array}{l}\vec{\infty} \\
\omega_{0}\end{array}$ & $\begin{array}{l}\stackrel{ }{N} \\
\text { ㅁ } \\
\dot{0}\end{array}$ & $\begin{array}{l}\circ \\
8 \\
\circ \\
\text { ㅇ } \\
\end{array}$ & $\begin{array}{l}8 \\
8 \\
=\end{array}$ \\
\hline 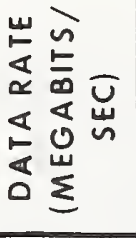 & $\stackrel{\circ}{\forall}$ & $\stackrel{\circ}{\circ}$ & $\begin{array}{l}\stackrel{0}{\sim} \\
\stackrel{\sim}{ }\end{array}$ & $\stackrel{\circ}{\circ}$ & "n & in & $\frac{0}{0}$ & $\begin{array}{l}\infty \\
\stackrel{+}{+} \\
\stackrel{0}{0}\end{array}$ & \begin{tabular}{l}
$\infty$ \\
\multirow{0}{0}{} \\
0 \\
0 \\
0 \\
0
\end{tabular} \\
\hline 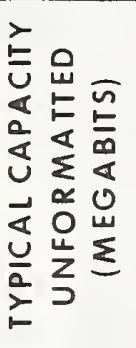 & $\stackrel{+}{m}$ & $\begin{array}{l}\circ \\
\circ \\
\circ \\
\circ \\
\circ \\
\vdots\end{array}$ & $\begin{array}{l}a \\
\infty\end{array}$ & $\begin{array}{l}0 \\
\stackrel{+}{\sim} \\
\sim \\
\sim\end{array}$ & $\begin{array}{l}0 \\
0 \\
0\end{array}$ & $\begin{array}{l}\infty \\
\dot{\sim} \\
\sim\end{array}$ & $\stackrel{\infty}{\sim}$ & $\stackrel{m}{\sim}$ & $\begin{array}{l}\nabla \\
\text { in }\end{array}$ \\
\hline 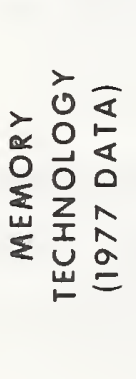 & 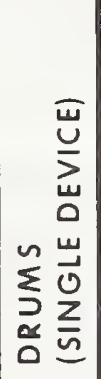 & 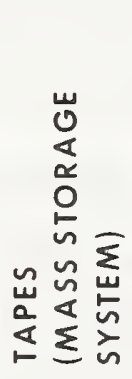 & 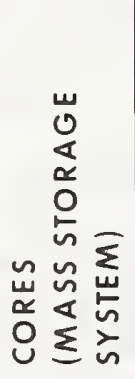 & 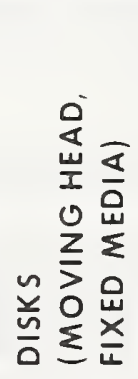 & 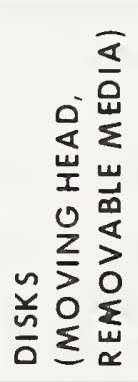 & 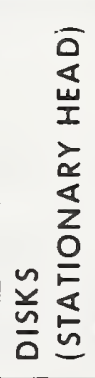 & 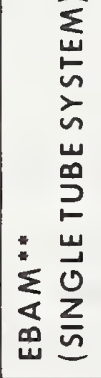 & 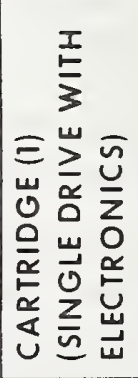 & 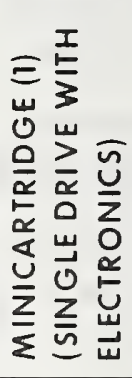 \\
\hline
\end{tabular}




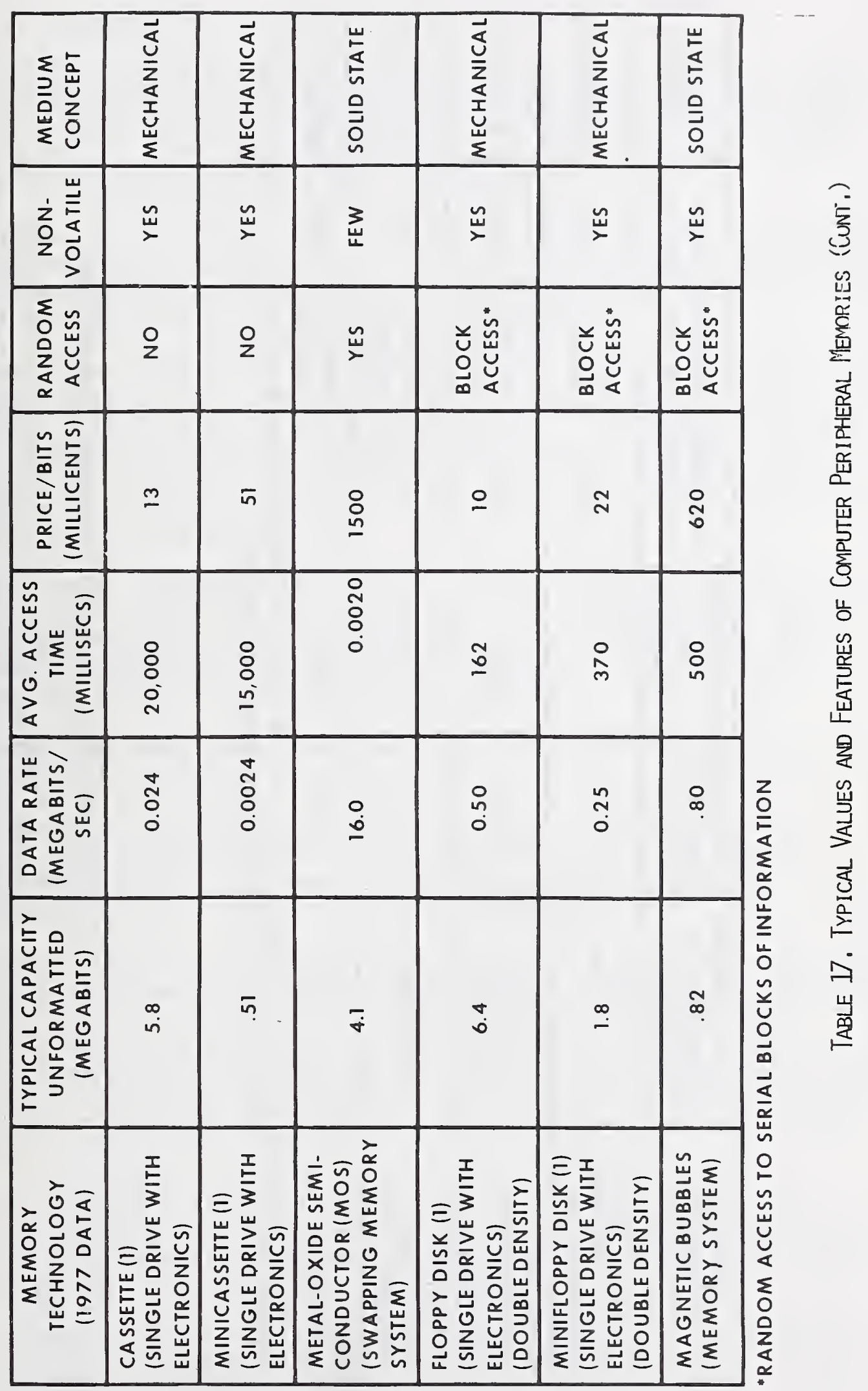




\begin{tabular}{|c|c|c|c|c|}
\hline 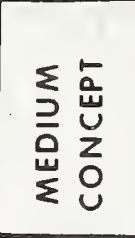 & 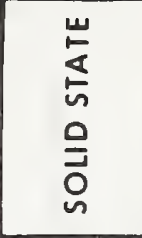 & 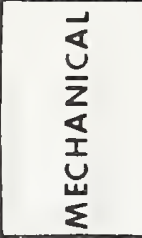 & 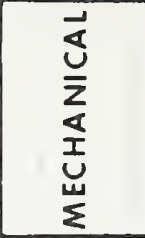 & 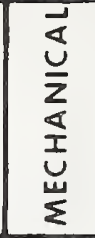 \\
\hline 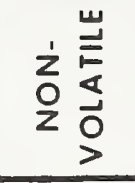 & 矛 & $\stackrel{\sim}{\sim}$ & $\stackrel{4}{\sim}$ & $\stackrel{\sim}{\sim}$ \\
\hline 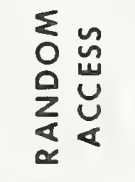 & 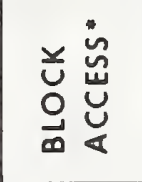 & 芯 & 总 & 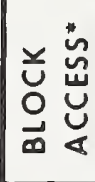 \\
\hline 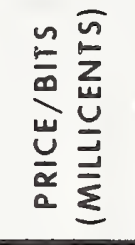 & $\begin{array}{l}\frac{0}{\alpha} \\
\text { à }\end{array}$ & $\begin{array}{l}\text { ㄱ. } \\
\text { ํ. } \\
\text {. }\end{array}$ & $\begin{array}{l}\text { ㅇ } \\
\text { ᄋ } \\
\text { ㅇ }\end{array}$ & 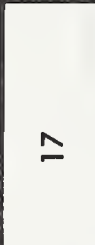 \\
\hline 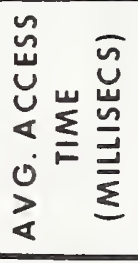 & $\stackrel{0}{\text { ㄴ. }}$ & $\begin{array}{l}\text { 은 } \\
\text { 은 }\end{array}$ & $\begin{array}{l}\text { ㅇ } \\
\circ \\
\text { о } \\
\text { ஸे }\end{array}$ & 응 \\
\hline 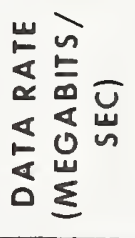 & $\stackrel{\circ}{\circ}$ & $\begin{array}{l}0 \\
0 \\
\text { in } \\
0 \\
0\end{array}$ & $\stackrel{\sim}{m}$ & $\stackrel{\circ}{-}$ \\
\hline 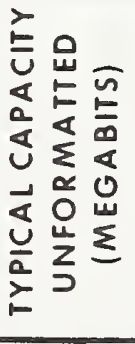 & $\underset{\infty}{+}$ & $\begin{array}{l}\circ \\
\circ \\
\circ \\
\circ \\
\circ \\
-\end{array}$ & $\begin{array}{l}\circ \\
8 \\
8 \\
8 \\
\circ \\
-\end{array}$ & $\underset{\sim}{\sim}$ \\
\hline 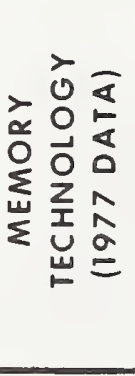 & 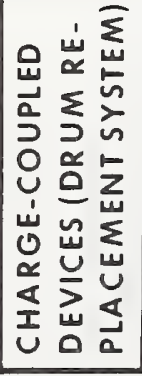 & 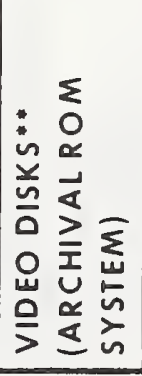 & 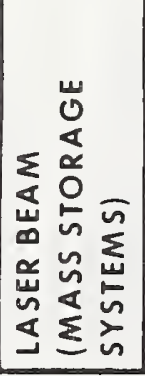 & 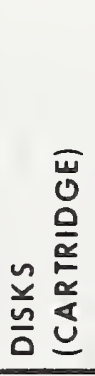 \\
\hline
\end{tabular}

है 


\section{B. Conclusions}

The following information is provided for the Federal computer manager as decision aids when purchasing, upgrading or leasing computer systems. In this report, the computer peripheral memory area is specifically addressed and we feel that it would be beneficial for Federal computer managers to consider the suggested approaches.

Approaches and suggestions are based on the information as presented in this report and are supported by a variety of views from sources such as manufacturers of computer systems and components, other forecasters and assessors, and private and Federal computer users.

Approaches and suggestions for choosing computer peripheral memory are presented in a series of decision trees as shown in Figure 12 through Figure 26. These decision trees represent most of the possible choices that can occur in the selection of peripheral memory hardware. Small computers are defined here as anything from micro-processors, microcomputers, and most minicomputers up to medium size computers -- large computers are those systems above this size category.

Guidelines are considered to be very general -- especially when new or emerging technologies, or those that are still seeking their market niche, are involved. Mostly, choices that are provided in the Figures are based on manufacturer's and user's suggestions and (projected) machine capabilities as represented in this report.

Important considerations in the selection of computer peripheral memory systems are as follows:

One especially important consideration is that final decisions of these kinds could be based on values other than technical; for example, established policies, economic factors, schedules, and personal preferences tend to play large roles in this process.

Also, any new memory technology must be at least as good as, or better than existing memories in terms of reliability, error rates, maintenance costs, initial price, etc., to be accepted -- and even then, manufacturers and users of proven technologies such as magnetic disk, core, and tape feel that their products will never completely disappear from the market because of continuous efforts to improve these technologies and simply because these systems have continued to survive for a long time.

Another frequently overlooked consideration is that any new memory subsystem must be fully developed in terms of hardware, software, interfacing, and fitting existing environments before users will consider purchasing it and installing it. [THEID 78]

And finally, users indicate that the particular application that needs to be filled most urgently might, and many times does become the most weighty factor in this decision process. 
As a rule of thumb, our guidelines mostly consider the equipment's cost/ performance factors and the timing in introducing the suggested peripheral memory systems. Many of the above-mentioned factors are left to the reader for additional considerations as might apply in individual circumstances.

The decision trees treat significant alterations of computer peripheral memories in the following manner:

- Upgrading

1. Large computer system

2. Small computer system

- Purchasing

1. Large computer system

2. Small computer system

- Leasing

1. Large computer system

2. Small computer system

Within each computer system, the following alterations are considered most common:

- Increase on-line capacity

- Increase off-line capacity

- Increase computer thruput via FAM memory

- Replace all, or some, of the existing memory

No information has been gathered in this report on the leasing of computer memory systems -- leasing should always be considered as a viable alternative to purchasing. This choice is presented because, in some cases, leasing could be advantageous for reasons such as possible better servicing, and economics.

In upgrading a large computer system, Federal computer managers should look at the following possibilities: (see Figure 12)

- Peripheral bulk core store

- Magnetic disk (moving head - fixed media)

- Tape mass storage systems

The independent bulk core store can help improve the accessibility of data to a speed not currently realized via other contemporary systems -manufacturers assure us that hardware, software, and other parameters look like fixed-head disks and should be transparent to the system. 


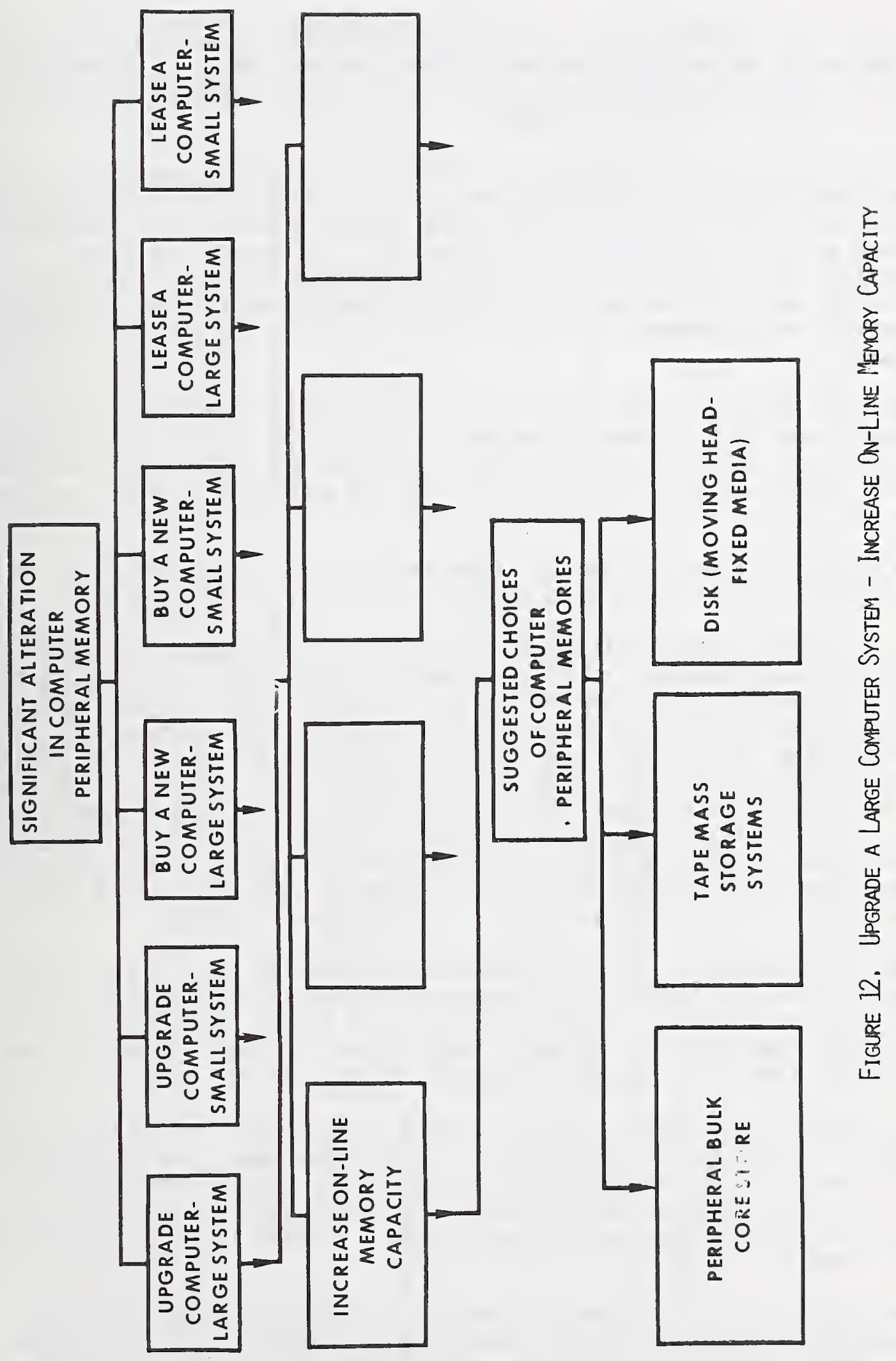


Moving-head, fixed media disks are good candidates when just a moderate increase in thruput and a relative higher storage capacity is required -all at a relative low price per bit. These systems have matured well and are considered very reliable.

Tape mass storage systems, up to one terabit, are being installed at many locations with apparently good success. When anticipating a very large data base requirement consisting of many records, these systems should prove to be a good choice. Several large manufactures are competing for a place in this market and many of these project that their systems will become very popular as the amount of on-line stored data becomes too great for conventional storage in the not too distant future.

Figure 13 shows that the best practice for storing off-line data on conventional large computer systems is still tape. Special mass storage systems are currently available that will load and operate with off-theshelf reels in tape libraries, while another manufacturer might provide its own type of reel that might be wider.

The costs for transferring data from one reel to another should be discussed in detail with each manufacturer.

For increasing computer thruput (if system is input/output (I/O) bound), fast auxiliary memories such as peripheral bulk core store or fixed-head disk can be considered (see Figure 14). Both systems will help improve $\mathrm{I} / 0$ transfer rates. The fixed-head disk and the core system, both priced about the same per bit, differ much in characteristics as can be seen in the data of Table 17. If fixed-head disks exclusively now make up the peripheral storage media, adding other disks might be considered best for fulfilling the new requirement; therefore, not affecting an already well-operating system. Possibly one may also look at a drum. In other situations, the bulk core system would probably do better and would have a vastly greater impact on the computer thruput.

Figure 15 suggests a situation wherein the existing gear needs to be replaced for reasons such as being worn out, being outdated, etc. The choice of replacement in this case could, of course, be extremely application sensitive. If an entire tape library system is involved, the automated tape mass storage systems should be considered.

For somewhat lower capacities but faster access time, moving-head disk systems are still very economical. On the other hand, manufacturers of bulk core store claim that their products would be more advantageous than fixed-head, fixed media disk in this case. But, when comparing these two systems core would be more costly. Possibly one may also look at a drum.

When upgrading a small computer system, as shown in Figure 16, it is assumed that peripheral storage capacity is generally moderate. This assumption introduces entirely new candidates for on-line storage that are usually priced lower. 


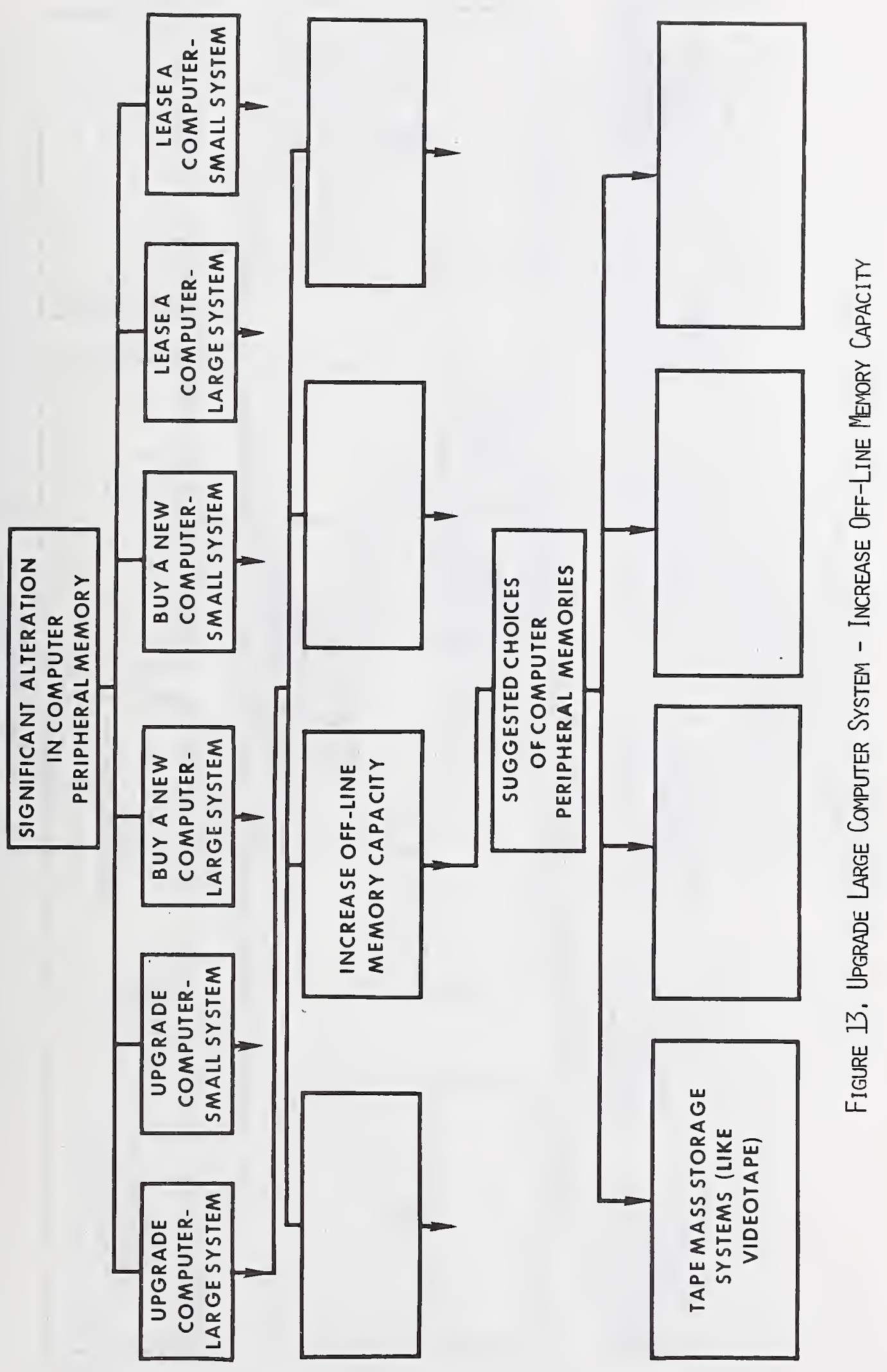




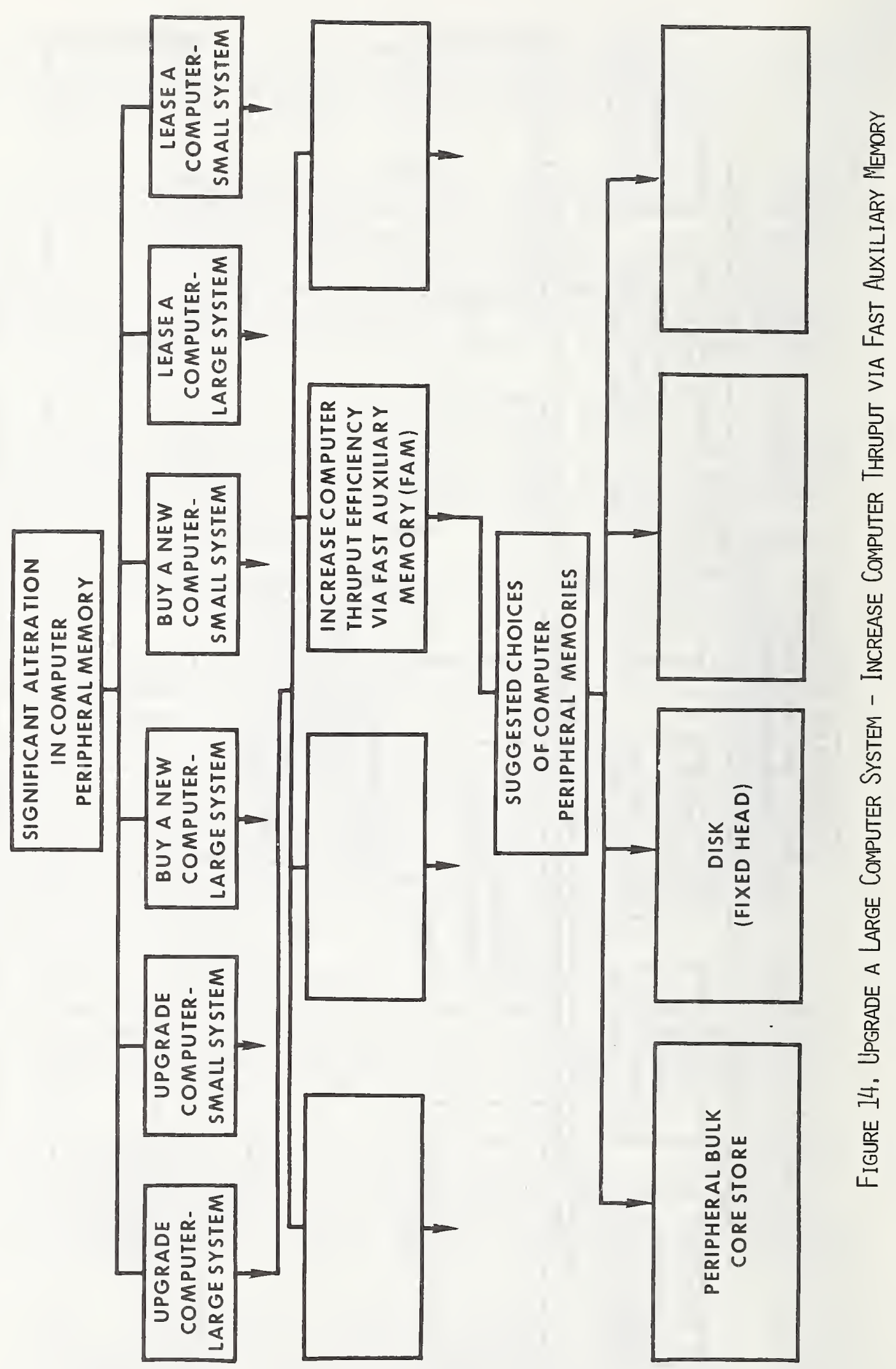




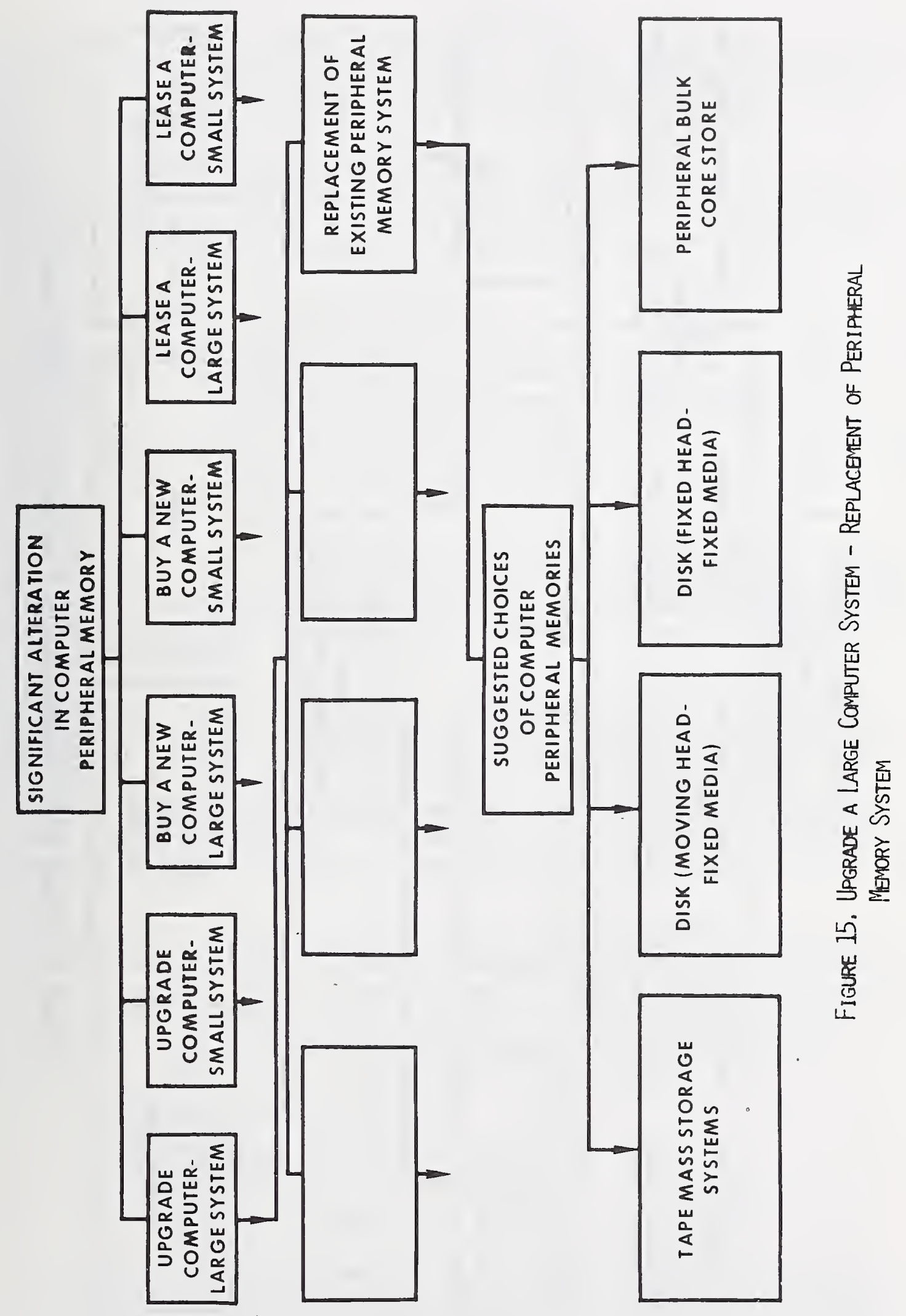




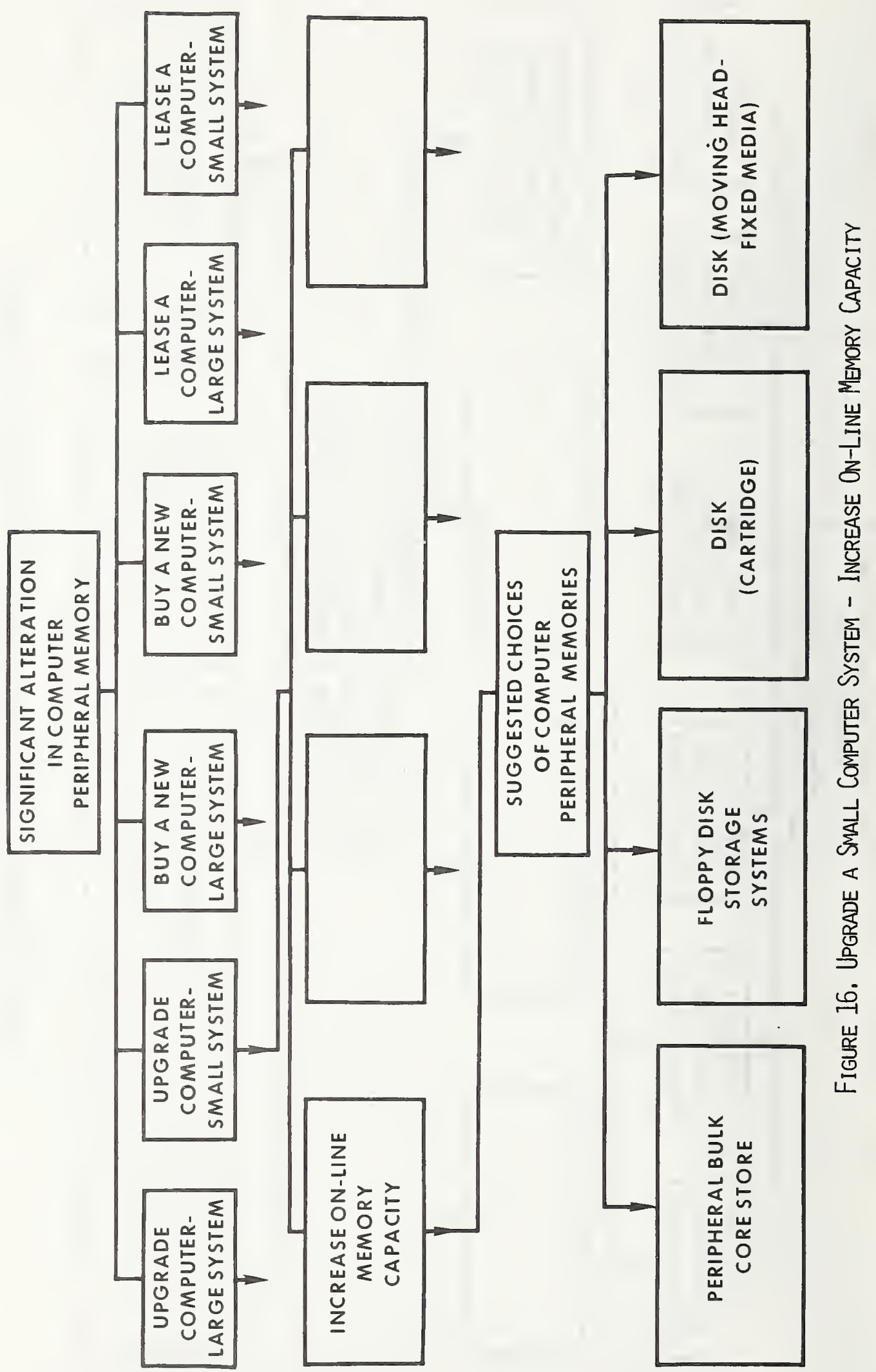


Peripheral bulk core could be considered but if a higher price is objectionable, and fast speed not needed, a floppy disk system might fit in as well. These systems have matured rapidly and are said to enjoy very good reliability at low price for both system and media.

Another consideration is magnetic disk, either a cartridge or movinghead magnetic disk system. A cartridge disk system would fit well with a small computer system because of small storage capacity, but the moving-head disk system would probably be more costeffective (see Table 17).

To increase off-line memory capacity of a small computer system, cartridge, cassette, and floppy disk storage systems should be considered (See Figure 17 for this decision tree). In cassette and cartridge systems, several sizes of the storage media such as standard or minicartridges and standard or minicassettes are available. In general, the cartridge checks out a little better as shown in Table 17 at about equal price per bit. However, in comparison the cassette system appears to be somewhat cheaper than the cartridge system.

If serial access is objectionable, the block-random accessed floppy disk system should be considered. It is about 200 times faster and slightly cheaper on a price per bit basis than the cassette or cartridge. Also, the data transfer rate of the floppy is better, about 10 times, than the serial tape units.

Figure 18 is the decision tree for increasing the thruput of a small computer system via a fast auxiliary memory (FAM). Two possible choices are offered -- the peripheral bulk core store and the fixed-head disk system. If fixed-head disks are already in use with the system and moderate upgrading is required, another fixed-head disk (or possibly a drum) could be considered. If considerable improvements are required, then the core system would be a better choice. Price per bit should be about the same.

Replacement of existing peripheral memory systems can be very much application dependent. Generally, replacement could be necessary due to wearout, increased requirements, change of mission, outdated equipment or other reasons. Replacement might not mean that equipment had to be upgraded. Perhaps, identical performance is desired at better reliability. There are various choices: (See Figure 19.)
Moving-head, fixed media disk
Floppy disk
Peripheral bulk core store
Cartridge disk
Magnetic bubble storage

For smaller capacities, consider floppy disks; and perhaps magnetic bubbles for special applications in hazardous, or secure environments. For larger capacities, look at moving-head disks and cartridge disks. 


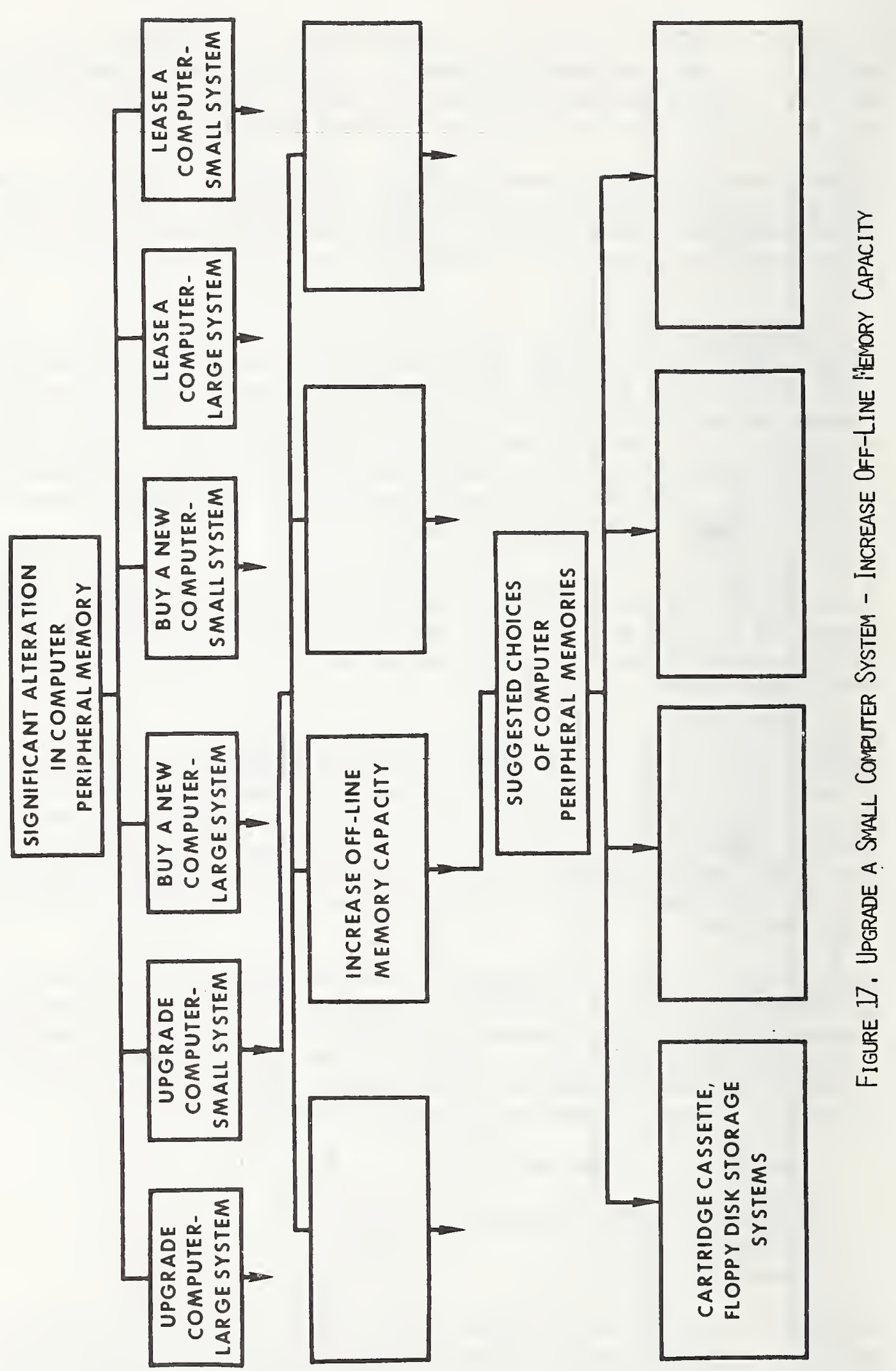




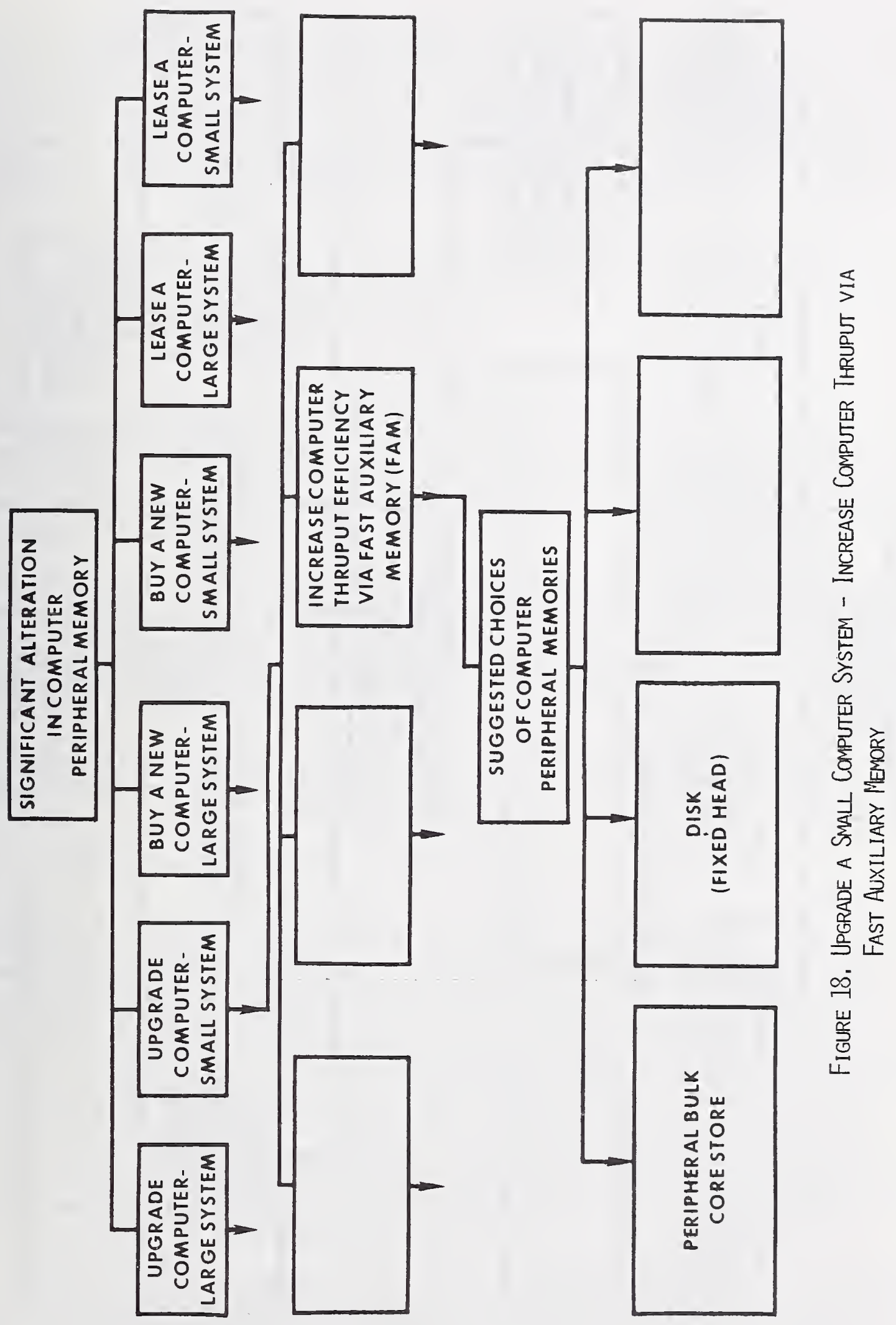




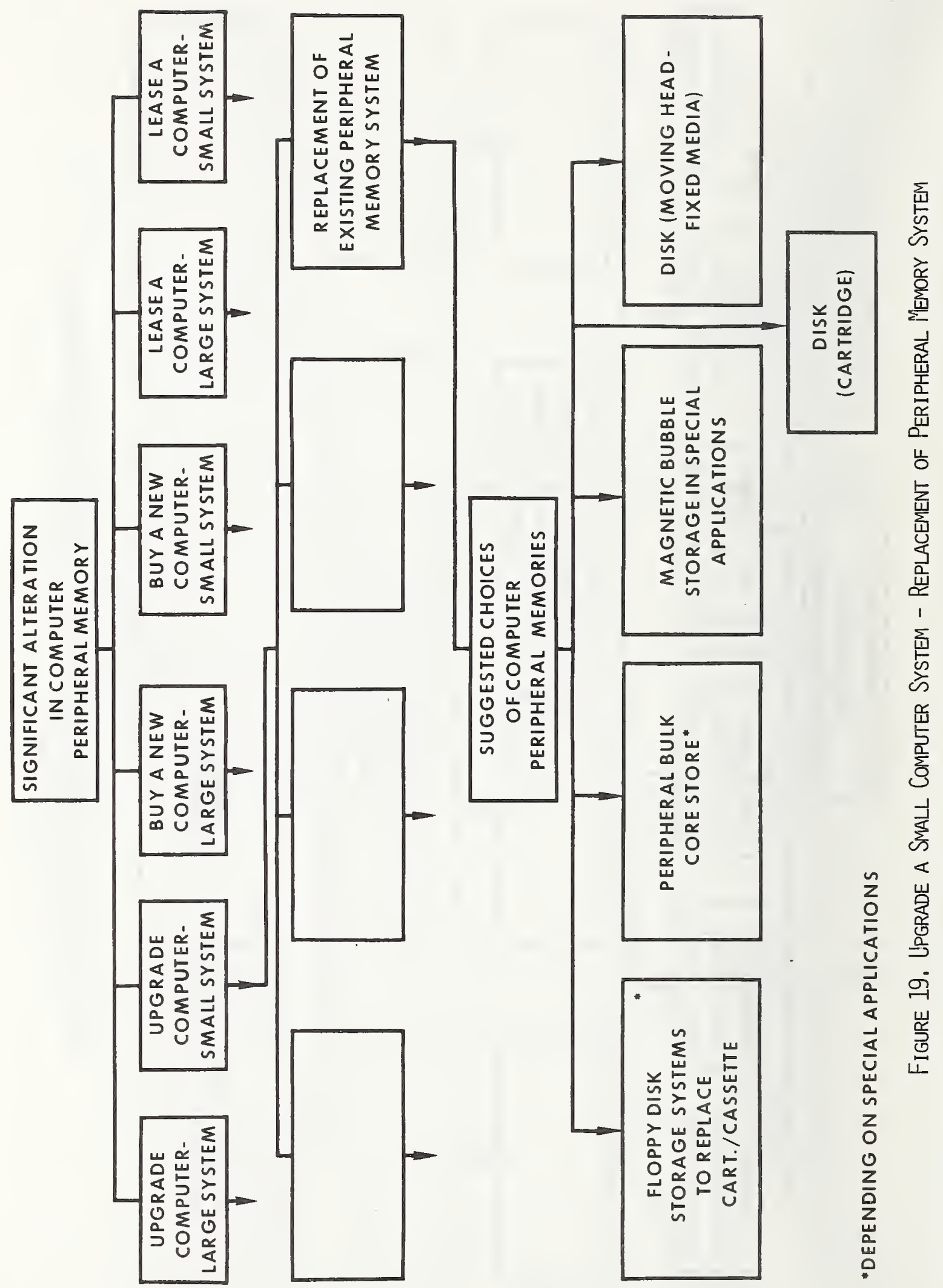


For special cases, where improvements in transfer rates and access times are requirements, the new peripheral bulk core stores should be good candidates. For the best price per bit, moving-head, fixed media disks are best, while the floppy disk system represents probably the cheaper system. When purchasing a new, and perhaps yet to be designed computer system, some of the following choices should be considered (See Figure 20).

\section{Charge-coupled device}

Magnetic disk (moving-head, fixed media)

Peripheral bulk core store

Tape mass storage

Laser mass memory

Optical videodisk memory (archival storage)

Charge-coupled devices are currently used as main memory extensions in new computer designs. This approach vastly increases the throughput of the machine at a reasonable increase in cost since CCD's are relatively low-cost memories when compared to conventional semiconductor randomaccess main memory.

For relatively low-cost, high capacity demands, moving-head disk memories are still good economical candidates. Bulk core store will provide a faster, relatively low-capacity storage system for meeting more stringent requirements. If very high capacity requirements are anticipated, the terabit tape mass storage systems should adapt well to new and future computers.

For extremely high-capacity storage, two types of optical mechanical mass store systems should be evaluated.

\section{Laser}

- Optical videodisk mass store

Laser mass store memories of up to one terabit are commercially available now -- optical videodisks are not (yet) available. Both of these read-only-memories (ROMs) store more information per unit volume than other mass storage systems and are more economical than these systems. Unlike soft magnetic records that can be easily erased, laser data is hard and any attempt at alteration can be detected. Laser-recorded data will not deteriorate or print through and is immune to electro-magnetic radiation and computer power failures. [MDATA 76A] Videodisks (see Table 17) are expected to have better memory operating characteristics than certain other laser memories; it is anticipated that these disks are targeted for archival storage applications. For future archival applications, either one of these systems should be considered as viable alternatives over tape archival systems.

off-line storage requirements for future computer systems in the form of archival, or read-only-memories, could be filled well by optical videodisk memories. The videodisk materials of these memories are very 


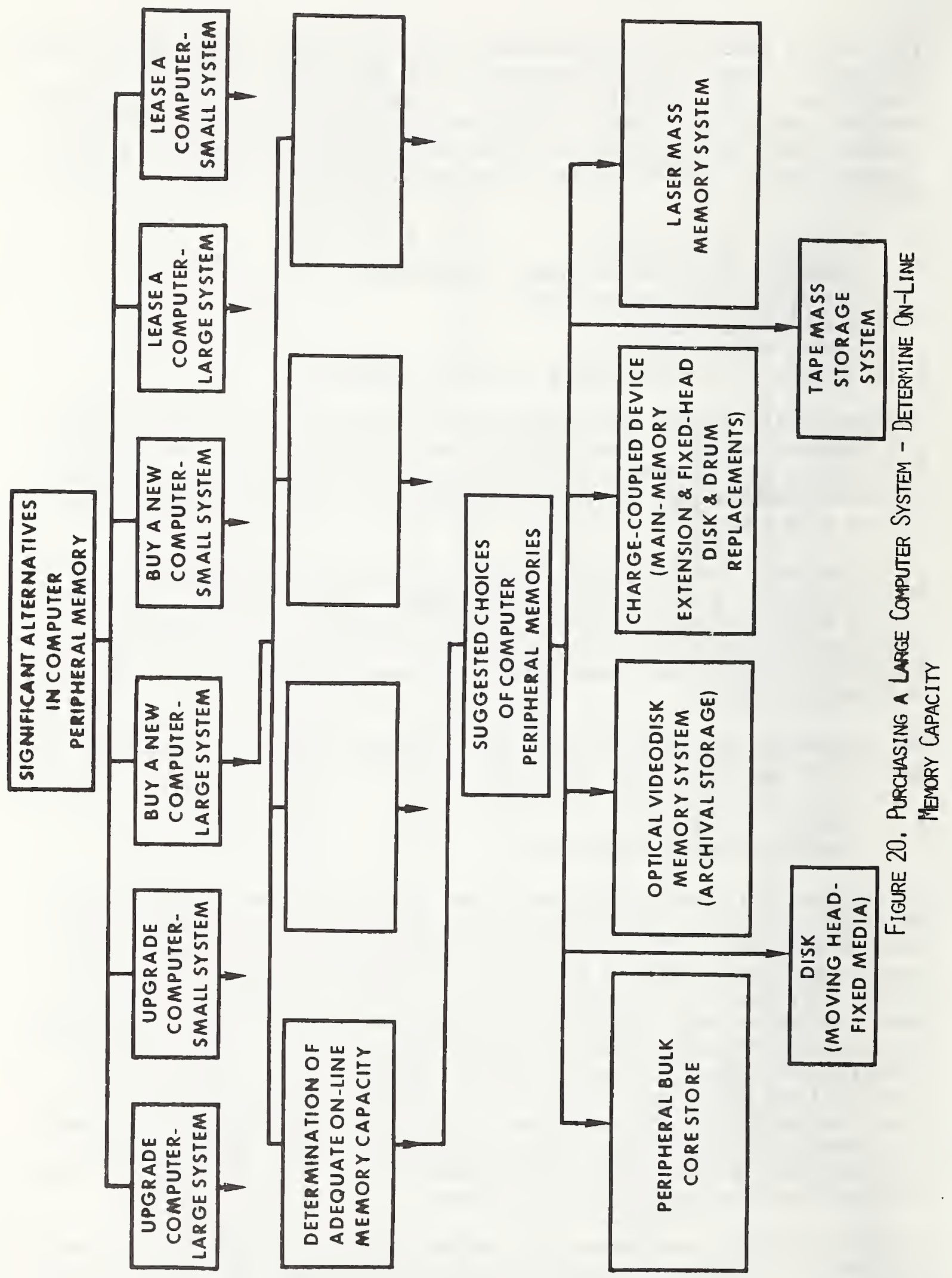


durable, rugged, and lend themselves to excellent portability. It would be excellent for off-line archival record storage.

Tape mass storage, claim many tape manufacturers, will always be around for future computers. And, it is very likely that videotape off-line mass storage will always be good for the recording and playback of large quantities of information. However, data retrieval will be slow and not as reliable as future optical memories; but, as opposed to videodisks, data can be erased. This could be an advantage or a disadvantage, highly dependent upon the application. Generally, most computer users feel that archival memories should not be erasable. The decision tree for this choice is shown in Figure 21 .

The choice of a fast auxiliary (FAM) memory for a large, new computer system is shown in Figure 22. A strong candidate to consider, in this case, is the electron beam addressable memory, or EBAM. A multiple tube system of this type for a future computer system would feature high capacity, nonvolatility, fast transfer rate, and fast access time, all at a reasonable price per bit if a relatively high capacity system (multiple tube) can be justified. This type of memory could improve the throughput of a large computer very noticeably.

Fixed-head, fixed media disks will still be available, according to their manufacturers, as another alternative. Possibly one may also look at drum memory. However, most computer users and manufacturers of other computer memories feel that these memories are on the decline in future applications.

When purchasing a small computer system, many choices in peripheral memories are available (see Figure 23). It is assumed that a small system does not need a vast peripheral memory capacity (although there are exceptions to this) and that some of these systems display operating characteristics such as fast access time, fast transfer rates, etc.

The choices are as follows:

- Charge-coupled device FAM

- Magnetic bubble device as nonvolatile special purpose mass storage Floppy disk storage system

Peripheral bulk core store FAM or special purpose mass store Moving head - fixed medium magnetic disk

Cartridge magnetic disk (small system)

Optical videodisk ROM mass store

According to users and manufacturers, two computer requirements are bound to increase. They are:

Memory capacity and data accessibility

System thruput

The use of CCD and bulk core store as part of the computer memory hierarchy can improve both of these capabilities at a potentially low-price 


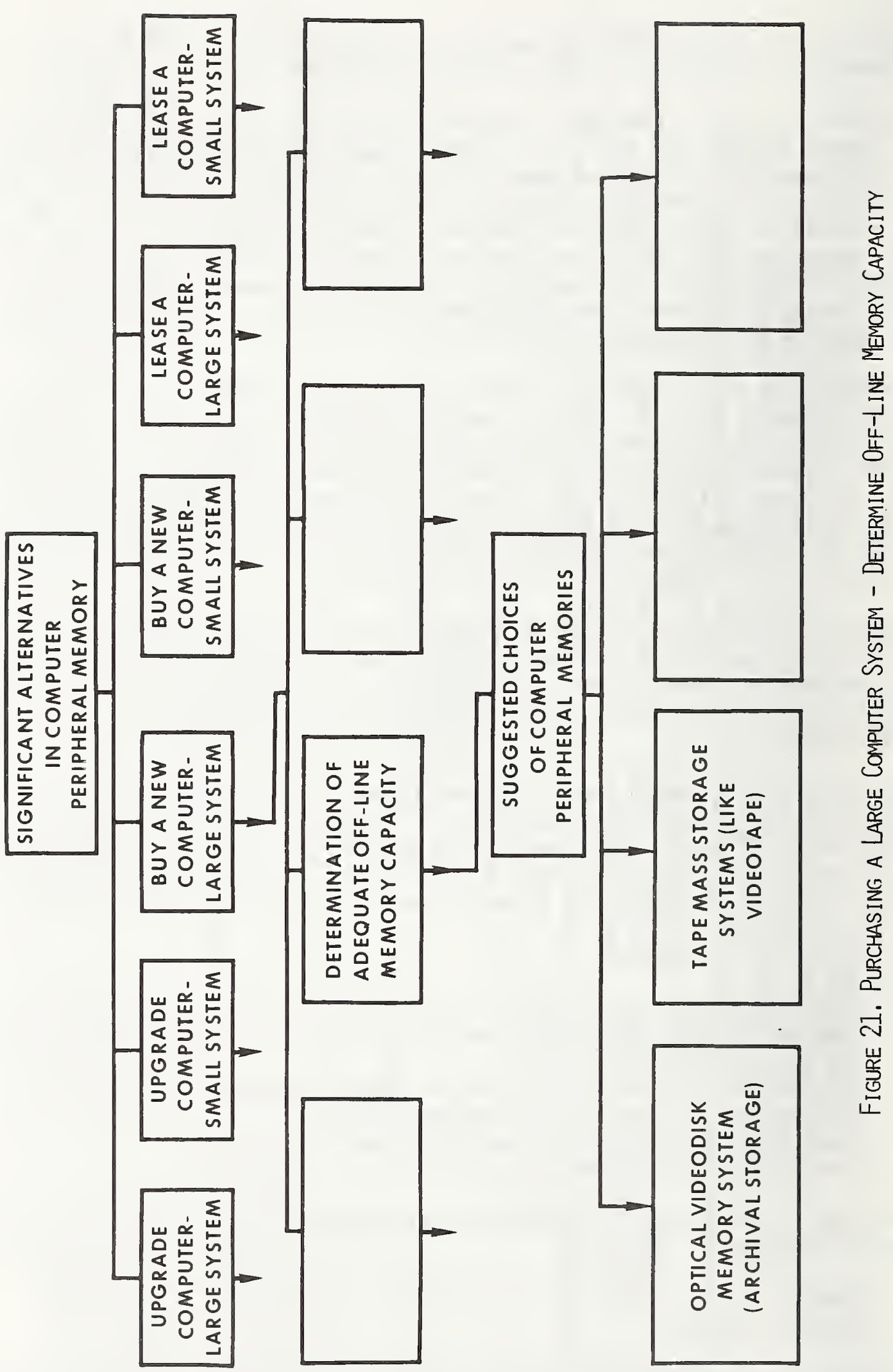




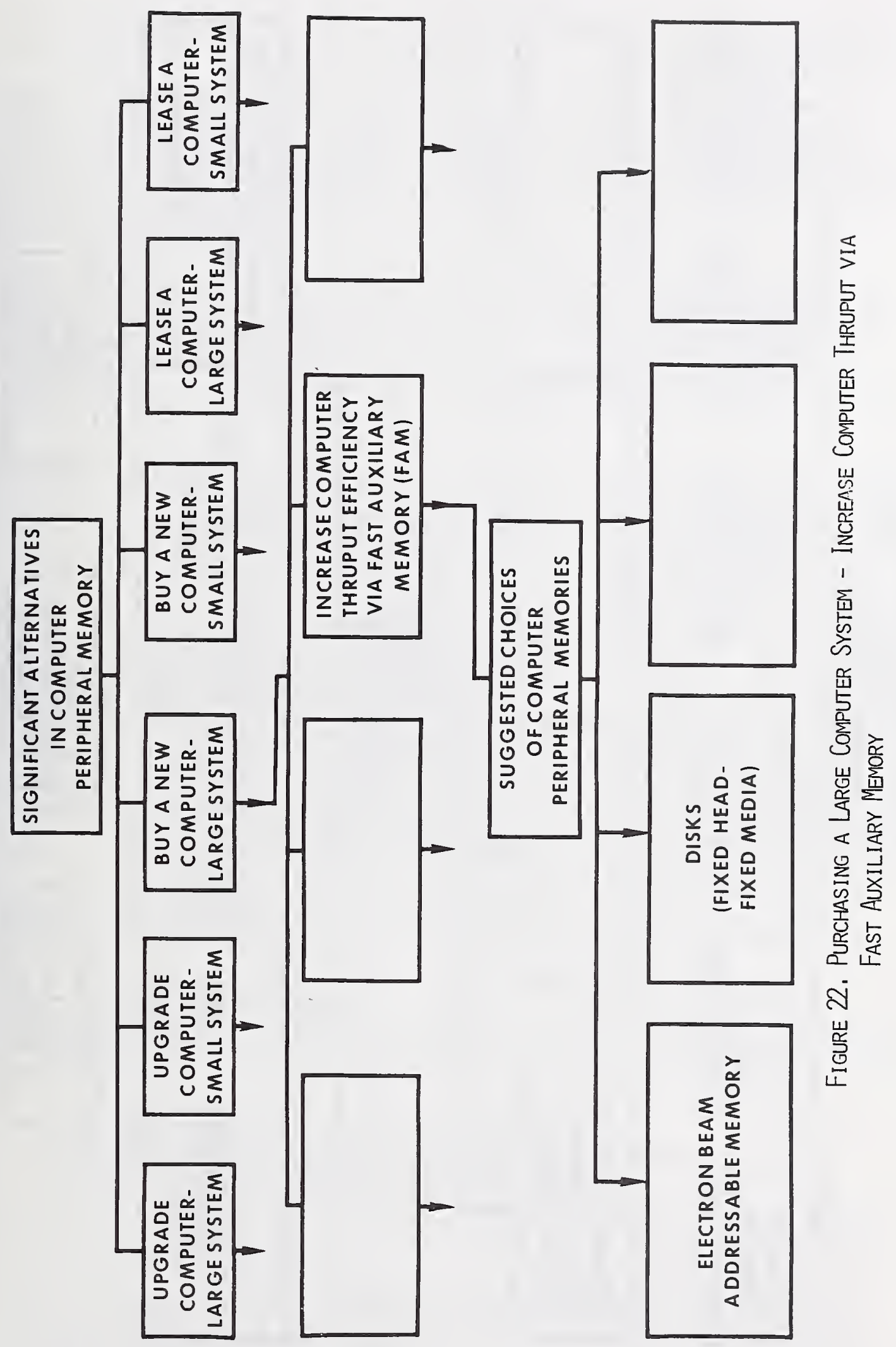




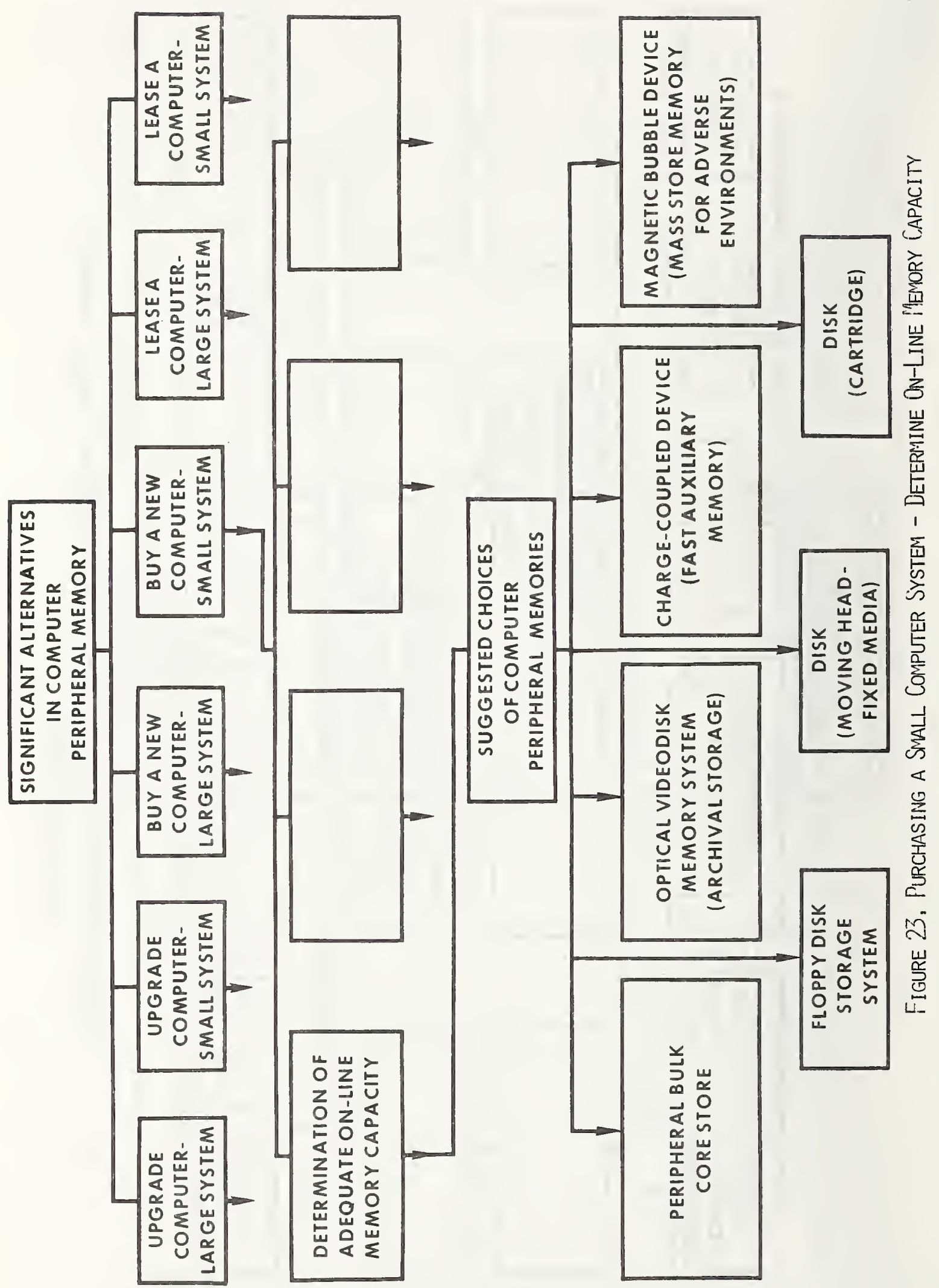


increase to the system. CCD's can be designed as part of the mainframe or can, like the bulk core, be applied as an "in-between" FAM memory.

Magnetic bubble mass store will outperform semiconductor and other magnetic memories in environments that need to be kept secure or are inherently hazardous in terms of radiation, shock or other adverse conditions. These memories are, therefore, also very useful in special applications since they are said to display excellent physical and electronic reliability.

Floppy disks can be used in areas where quick data entry of small records is routine. Some designs also use these disks as on-line memories (usually in very small systems). Floppy disks are excellent recordkeepers, and they are easily stored. The medium cost is very attractive and, since system cost is also very reasonable, many applications should be shifted towards the floppy.

For larger and faster (and more expensive) on-line storage, moving-head, fixed medium disks and the lower capacity (but cheaper) cartridge disk systems are good candidates for future, small computer systems. These units can be staged through the previously mentioned FAMs (CCD and bulk core store) for forming a fast I/O transfer peripheral system.

Optical videodisk memories of the future should lend themselves very easily to both large and small computer systems. This is said to be so since video disk memories are relatively easy to operate and since their volumetric memory efficiency is excellent (one terabit is said to be portable). Of course, this would be a read-only application. When deciding on videodisks, one should remember that, even though these systems are ROMs, R/W operations can still be performed for many applications. For example, in recordkeeping and updating, enough "freespace" writing area can be allowed to satisfy the bookkeeping of most records. And since the medium is cheap, takes up little space and does not wear, old records can be kept on-line virtually indefinitely at little or no extra cost. Of course, it must be kept in mind that a REWRITE over already written data is not possible in this technology, as yet.

Figure 24 shows some of the possibilities that exist for choosing offline storage for a new, small computer system design. The choices are as follows:

Cartridge, cassette, and floppy disk

- Magnetic bubble device (special off-line recorder)

- Optical videodisk read-only memory system

The main off-line storage in most minicomputers will be on cartridges, cassettes, and floppy disks.

Magnetic bubbles could, according to some forecasters and MB manufacturers, compete and be used as fast solid-state floppy disk substitutes in special applications. 


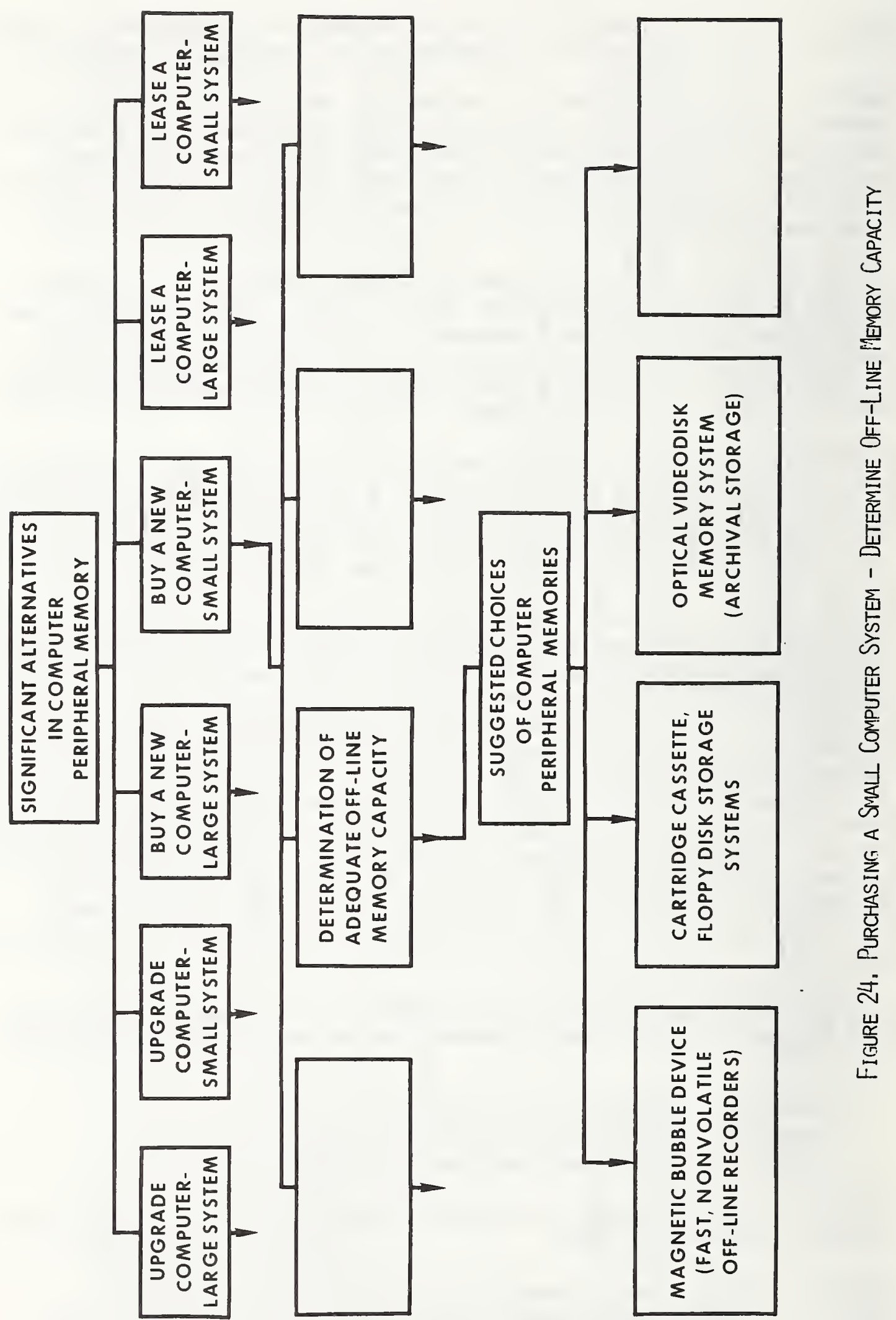


Optical videodisk memories, when available, will offer a significant off-line storage back-up to the small computer system. Their vast data storage capacity, portability, ruggedness, and fast access should make these memories particularly useful in applications such as video recordkeeping, digital storage, education, training, graphics, and equipment manual data presentations.

Figure 25 is the decision tree for choosing which peripheral memory system might serve best as a fast auxiliary memory in an effort to increase the system I/O thruput. The choices presented are:

- Charge-coupled device

- Magnetic bubble device (advanced, high-speed designs)

- Peripheral bulk core store

- Fixed head - fixed medium disk or possibly also a magnetic drum (in special applications)

Of these choices, it is anticipated that CCDs and peripheral bulk cores will be the better developed systems -- their speed, system size, power consumption and, hopefully, economics will make them quite attractive in this application.

Magnetic bubble memories will find their place first in special applications as auxiliary memories -- and as their speed, price and designs mature, they will start to compete with the magnetic disk memory systems in the near future.

Fixed-head, fixed medium disks might be used in very special applications, but it is anticipated that the demand for these units in this particular application, as well as others, will diminish rapidly in the near future. 


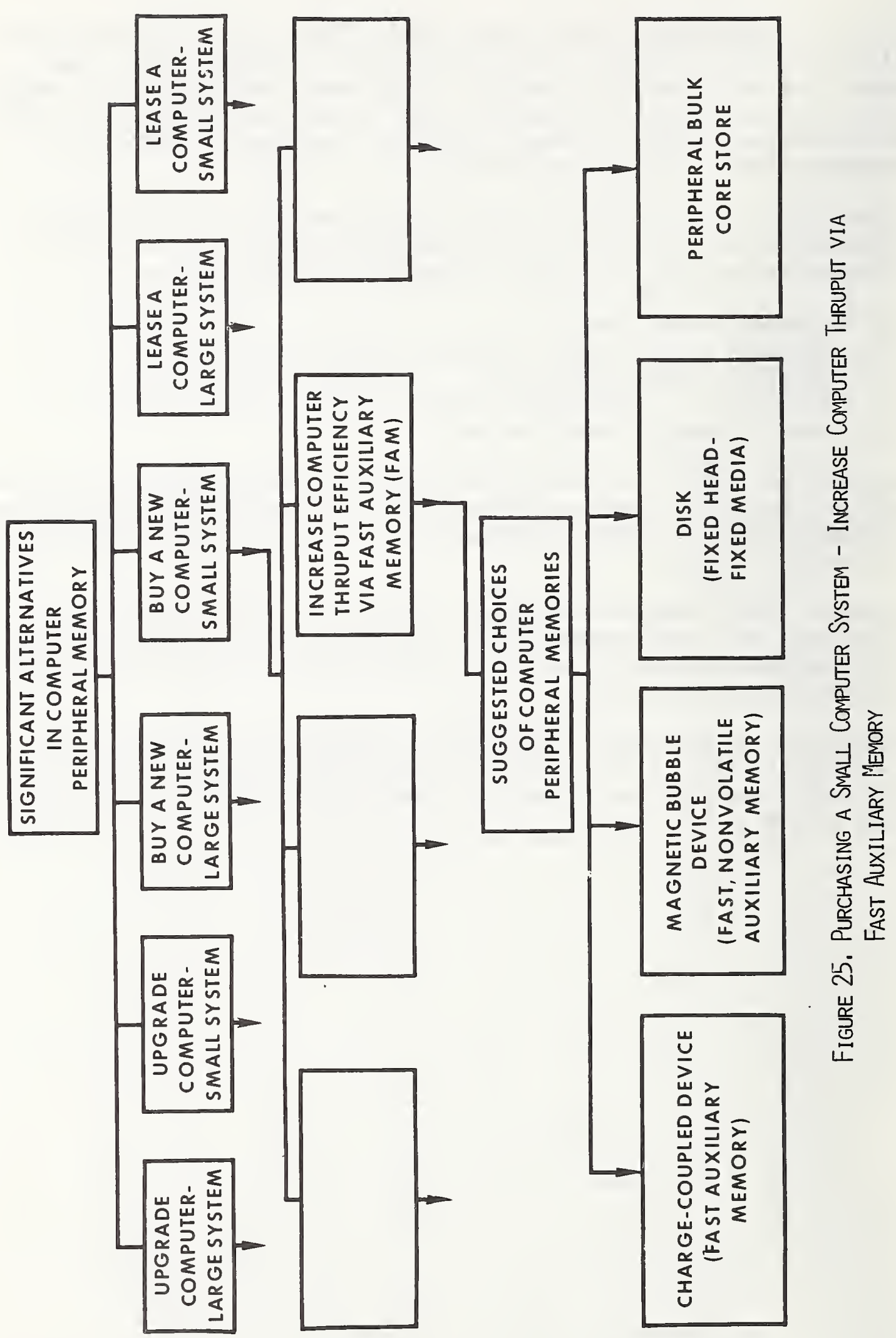




\section{Bibliography}

[ALPHA ND] Alpha Data Incorporated. CCDISC memory system. Chatsworth, CA; [n.d.]. [2 p.].

[AMPEX ND] Ampex Corporation Memory Products Division, 1223 megastore. El Segundo, CA; [n.d.]. [6 p.].

[ATKIN 75] Atkinson, Norman. Small Storage Peripherals: Floppy disc. Data Processing, 1975 January/February; 17(1): 48.

[AUERB 75A] Auerbach Computer Technology Reports. California Computer Products 7110 Automated Tape Library; Philadelphia, PA; Auerbach Info, Inc.; 1975 June; 220.2007.060. 1-3 p.; [n.a.].

[AUERB 75B] Auerbach Computer Technology Reports. Control Data: 38500 Mass Storage System; Philadelphia, PA; Auerbach Info, Inc.; 1975 October; 220-2952.120. 3 p.; [n.a.].

[AUERB 75C] Auerbach Computer Technology Reports. Ampex Corp. TBM Mass Storage System; Philadelphia, PA; Auerbach Info, Inc.; 1975 December; 220-1276. 100.1-4 p.; [n.a.].

[AUERB 75D] Auerbach Computer Technology Reports. System 190 Laser Mass Memory; Philadelphia, PA; Auerbach Info, Inc.; 1975 December; 220.5520.180.1-3 p.; [n.a.].

[BACKJ 75] Backler, Jordan. Floppies grow Flexible. Minicomputer News. 1975 April; 10:6.

[BACKJ 76] Backler, Jordan. Low Cost Data Storage Multiple Options-Hard Choices. Digital Design. 1976 June; 6(6): 60-70.

[BARCM 77] Barclay, Mike. Discs - past, present and future. Data Processing. 1977 May; 19(5): 29,32.

[BARNS ND] Barnes, Sam. Elastomeric Band Simplifies Tape Drive. Reprinted from Design News. [n.d.]. 1 p.

[BASF ND] Badische Anilin - \& Soda - Fabrik AG [BASF]. BASF Disk Packs - perfect - reliable. 6700 Ludwigshafen AM Rhein, West Germany. [n.d.]. [6 p.].

[BHAND 78] Bhandarkar, Dileep P. Dynamic MOS Memories: Serial or Random Access? Spring Compcon 78 Sixteenth IEEE Computer Society International Conference; 1978 February 28-March 3; San Francisco, CA. New York; IEEE Computer Society; c 1978: 162-164.

[BLAKT 75] Blakeslee, Thomas. Powering Up a Cartridge Tape Drive. Digital Design. 1975 February; 5(2): 28-32, 38. 
[BOWED 75] Bowers, Dan M. The Computer Memory Market. Modern Data. 1975 August; 8(8): 51.

[BORKA 77] Bork, Alfred M. Videodiscs - The Ultimate Computer Input Device? Creative Computing. 1977 March-April; 2(2): 44-45.

[BRECR 78] Brechtl'ein, Rick. Comparing Disc Technologies. Datamation. 1978 January; 24(1): 139-150.

[BRIGG ND] Briggs, G. A. Sound Reproduction. IDLE, Bradford Yorks: Wharfedale Wireless Works; [n.d.]. 264-273.

[BRITA 74] Gutenberg, Johannes. The New Encyclopaedia Britannica: in 30 volumes. Chicago, London, Toronto, Geneva, Sydney, Tokyo, Manila, Seoul, Johannesburg: Encyclopaedia Britannica, Inc.; 1974: 505-506.

[BUSIN 76] The Floppy Disk Gets a Whirl. Business Week. 1976 May 17; 2432:44AA-44DD.

[BUSIN 77] The Bubble Memory Finally Arrives. Business Week. 1977 March 28; 2476: 72, 74 .

[BUSIN 78] Semiconductors: A Sure Bet For First-Half Growth. Business Week. 1978 January 9; 2516: 55-58.

[BUTLM 76] Butler, Margaret K. Prospective Capabilities in Hardware. AFIPS Conference Proceedings; 1976 June 7-10; New York City, New York. Montvale: AFIPS Press; c 1976: 323-336.

[BYTE 76] State of the Art Disk Technology. BYTE. 1976 December; 16: 86-87.

[CALIF 76A] California Computer Products, Inc. 140 Floppy Disk Drive. Anaheim, CA; 1976. [2 p.]; SS964A 5M 676.

[CALIF 76B] California Computer Products, Inc. (CALCOMP). 7110 Automated Tape Library. Anaheim, CA; 1976; [2 p.]; SS947E 5M 1076.

[CALIF 77] California Computer Products, Inc. $142 \mathrm{M}$ Floppy Disk Drive. Anaheim, CA; 1977; [2 p.] SS987 5M 277.

[CALOP 77A] Calomeris, Peter J. (Interviewer). Interview with Floppy Disk Manufacturer. 1977 May 25.

[CALOP 77B] Calomeris, Peter J. (Interviewer). Interview with Floppy Disk Manufacturer. 1977 May 27.

[CALOP 78A] Calomeris, Peter J.; Warnar, Robert B. J.; Recicar, Steve A. Interview with Laser Mass Memory Manufacturer. 1978 March 29. 
[CALOP 78B] Calomeris, Peter J. Compilation of Floppy Disk Drive Market Data: Calculating the Average. 1978. Unpublished draft.

[CAPER 77] Capece, Raymond P. Memory-Oriented Designs Maximize Throughput. Electronics. 1977 October 27; 50(22): 104-110.

[CARSR 73] Carson, Robert W. Minicomputer Removable Storage. Modern Data. 1973 March; Special Report; 5 p. Available from: Data Products, 3M Company, Camarillo, CA.

[CASWS 75A] Caswell, Stephen A. Floppy Disk Drives and Systems. Modern Data. 1975 August; 8(8): 32-41.

[CASWS 75B] Caswell, Stephen A. Tape Cartridge Drives and Systems. Modern Data. 1975 September; 8(9): 35-44.

[CASWS 75C] Caswell, Stephen A. Cassette Drives and Systems. Modern Data. 1975 October; 8(10): 58-63.

[CDC ND] Magnetic Peripherals Inc. Control Data Corporation. Memory. Minneapolis, MN; [n.d.] [4 p.].

[CEN 77] Technology: Two New Computer Terminals Using Bubble Memories Have Been Developed. C \& EN. 1977 April 25; 55(17): 21.

[CHACP 72] Chacedhari, P. Defects in Garnet Suitable for Magnetic Bubble Domain Devices. IEEE Transactions on Magnetics. 1972 September; 8: 333-339.

[CHANH 75] Chang, Hsu. Magnetic Bubble Technology: Integrated Circuit Magnetics for Digital Storage and Processing. New York: IEEE Press: 1975: 15, 17, 20, 21, 39, 89, 215-218.

[CHEND 75] Chen, Di; J. David Zook. An Overview of Optical Data Storage Technology. Proceedings of the IEEE. 1975 August; 63(8): $1207-1230$.

[CHRISM 77] Christopher, Maxwell. Interview with Laser Mass Memory Manufacturer. 1977 June 21. 3 p.

[COMP 76] Compact Data Cartridge System Offered by 3M Computer. 1976 March. $9(3): 57$.

[COMPD 77] Mass and Mini Storage: Storage Devices for Both Mainframes and Minis are Improving in Speed, Capacity and Reliability. New Technologies will Increase Their Performance Even More. Computer Decisions. 1977 March; 9(3): 48-52, 54.

[COMPU 75] Mass Storage System Moves Data Directly from Cartridge to Computer. Computer Design. 1975 July; 14(7). 18. 
[COMPU 76A] Bulk Core System Bridges Gap Between Main Memory and I/0 Devices. Computer Design. 1976 May; 15(5): 22.

[COMPU 76B] Small Floppy Disc Drive Meets Microcomputer System Requirements. Computer Design. 1976 September; 15(9): 134, 136.

[COMPU 77A] Core-Based Mass Storage System Replaces Discs, Lowers Access Time. Computer Design. 1977 March; 16(3): 27.

[COMPU 77B] Nine New Memories from the Leader...Texas Instruments. Computer Design. 1977 April; 16(4): 40-41.

[COMPU 77C] Interchangeable 4K/]6K RAMs Can Reduce Cost of Systems Design. Computer Design. 1977 May; 16(5): 152.

[COMPU 77D] Replacement for Rotating Disc Memories Based on CCDs. Computer Design. 1977 May; 16(5): 22.

[COMPU 77E] Single-Board Memory Stores 128K-Bytes with Error Correction. Computer Design. 1977 May; 16(5): 22.

[COMPW 75] Micos System Gets Swapping Memory. Computerworld. 1975 June $25: 29$.

[COMPW 77] Fairchild Offering CCD Memory Sample. Computerworld. 1977 March 21: 42 .

[CONWJ 77A] Conway, John. Special Report: Flexible Discs vs. Magnetic Tape - Are They Complementary or Competitive? EDN. 1977 February $5 ; 22(3): 36-46$.

[CREAT 76] How the Videodisc Players Work. Creative Computing. 1976 March-April; 2(2): 42-43.

[CUMMM 75] Cumming, Mike. Small Storage Peripherals: Cartridge Disc. Data Processing. 1975 January/February; 17(1): 44-45.

[DATMI 74] A Mass Market for Mass Storage? Datamation. 1974 November; 20(11): 118-120.

[DATMI 76] Erasing Myths about Magnetic Media. Datamation. 1976 March; $22(3) ; 65-70$.

[DATMI 77] Floppies Continue Their Price Dive. Datamation. 1977 May; $23(5): 253-254$.

[DATPC 75] Floppy Disc as Input Medium. Data Processing. 1975 January/February; 17(1): 40-42.

[DATPD NDA] Dataproducts. Dataproducts store/3220 core memory system, the $32 \mathrm{~K}$ jewel. Woodland Hills, CA; [N.D.]. [6 p.] 
[DATPD NDB] Data Products 3M Company. In the Grand Tradition of the Paper Clip. Camarillo, CA; [n.d.] [2 p.].

[DATPN 77] Fast-Access storage with CCDs. Datapro Newscom. 1977 April; 5(4):4 p.

[DATPR 76] Datapro reports on Minicomputers. Minicomputer Cassette and Cartridge Magnetic Tape Units; Delran, NJ; 1976 Datapro Research Corporation; 1976 May;M13-100-351 to M13-100-354 (Updated Reference Source).

[DATPR 77A] Datapro reports on Minicomputer. Wangco/Orbis Model 82 MicroFloppy; Delran, NJ; 1977 Datapro Research Corporation; 1977 January; Ml3-100-272 (Update Reference Source).

[DATPR 77B] Datapro reports on Minicomputers. Advanced Electronics Design AED2200; Delran, NJ; 1977 Datapro Research Corporation; 1977 October; M13-100-209 (Update Reference Source).

[DATPR 77C] Datapro reports on Minicomputers. Control Data 9427H; Delran, NJ; 1977 Datapro Research Corporation; 1977 0ctober; M13-100-215 (Update Reference Source).

[DATPR 77E] Datapro reports on Minicomputers. Diablo Model 31; Delran, NJ; 1977 Datapro Research Corporation; 1977 October; Ml3-100-221 (Update Reference Source).

[DATPR 77F] Datapro reports on Minicomputers. Minicomputer Disk Storage; Delran, NJ; 1977 Datapro Research Corporation; 1977 October; M13-100-225, M13-100-228, M13-100-242 (Update Reference Source).

[DATPR 77G] Datapro reports on Minicomputers. Pertec D3311; Delran, NJ; 1977 Datapro Research Corporation; 1977 0ctober; Ml3-100-235 (Update Reference Source).

[DATPR 74] Datapro 70 the EDP Buyer's Bible. IBM system/360 Model 22-195: Delran, NJ: 1974 Datapro Research Corporation; 1974 January; 70C-49l-03e to 70C-491-03g, 70C-49]-05h. (Updated Reference Source).

[DATPR 75] Datapro 70 The EDP Buyer's Bible. Control Data 38500 Mass Storage System; Delran, NJ; 1975 Datapro Research Corporation; 1975 September; 70D-263-2la to 70D-263-2ld. (Updated Reference Source).

[DATPR 76] Datapro 70 the EDP Buyer's Bible. IBM System/370; Delran, NJ; 1976 Datapro Research Corporation; 1976 April; 70C-491-04x, 70C-491-04y. (Updated Reference Source). 
[DATPR 77A] Datapro 70 The EDP Buyer's Bible. Control Data Cyber, 170 Series; Delran, NJ; 1977 Datapro Research Corporation; 1977 June; 70C-263-12h to 70C-263-12i, 70C-263-12u. (Updated Reference Source)

[DATPR 77B] Datapro 70 the EDP Buyer's Bible. IBM System/370; Delran, NJ; 1977 Datapro Research Corporation; 1977 April; 70C-491-04w to 70C-491-04x. (Updated Reference Source).

[DATPR 77C] Datapro 70 The EDP Buyer's Bible. UNIVAC 1100 Series; Delran, NJ; 1977 Datapro Research Corporation; 1977 January; 70C-877-1la to 70C-877-1lgg. (Updated Reference Source).

[DATQS 76] Electronic Memories: Charge Coupled Devices and Magnetic Bubble Devices. Dataquest Research Newsletter. 1976 November $5 ; 1-7$.

[DATQS 77] MOS Memory - 4K Shipments. Dataquest Research Newsletter. 1977 March 25: 1-5 p.

[DATQS 78] MOS Memory - 4K and 16K Shipments. Dataquest Research Newsletter. 1978 February 3: 1-6 p.

[DATRM NDA] Dataram Corporation. Add-in Memory for PDP-8/E,F and M. Cranbury, NJ; [n.d.]. [2 p.].

[DATRM NDB] Dataram Corporation. Add-in Memory for Varian V70 Series. Cranbury, NJ; [n.d.]. [2 p.].

[DATRM NDC] Dataram Corporation. Add-on/Add-in Memory for DEC PDP-11. Cranbury, NJ; [n.d.]. [2 p.].

[DATRM NDD] Dataram Corporation. Add-on Add-in Mini-Memory, Cranbury, NJ; [n.d.]. [l p.].

[DATRM NDE] Dataram Corporation. Bulk core. Consider the implication of this perspective twisting new product from Dataram Corporation. Cranbury, NJ; [n.d.]. [1 p.].

[DATRM NDF] Dataram Corporation. Bulk Core Replacement for Data General Novadisc. Cranbury, NJ; [n.d.]. [2 p.].

[DATRM NDG] Dataram Corporation. Bulk Core Replacement for DEC RC-11 and RF-1l Fixed-head disk systems. Cranbury, NJ; [n.d.]. [2 p.].

[DATRM NDH] Dataram Corporation. The DR-100 Family core memory systems. Cranbury, NJ; [n.d.]. [6 p.].

[DATRM NDI] Dataram Corporation. Interdata-compatible Add-in Memory. Cranbury, NJ; [n.d.]. [2 p.]. 
[DATRM NDJ] Dataram Corporation. A New high in storage capability bulk core. Cranbury, NJ; [n.d.]. [8 p.].

[DATRM NDK] Dataram Corporation. Nova-Compatible Add-in Memory. Cranbury, NJ; [n.d.]. [2 p.].

[DATRM NDL] Dataram Corporation. Replace Fixed-Head Disc with Dataram Bulk Core. Cranbury, NJ; [n.d.]. [] p.].

[DATRM NDM] Dataram Corporation. 1K to 32K Bytes Microprocessor Core Memory. Cranbury, NJ; [n.d.]. [2 p.].

[DAVIP 76] Davies, Paul. Disk Cartridge Driver. Modern Data. 1976 January; $9(1): 44,45$.

[DAVIS 74] Davis, Sidney. Update on Magnetic Tape Memories. Computer Design. 1974 August; 13(8): 127-138.

[DIGID 75A] Electron Beam Memory Stores 32M Bits. Digital Design. 1975 June; 5(6): 15-18.

[DIGID 75B] Hard-disk drives. Digital Design. 1975 December. 15(12): 59.

[DIGID 76A] Cart/Cassette Drives. Digital Design. 1976 January; 6(1): 67.

[DIGID 76B] Moving-head Disks at Performance Plateau? Digital Design. 1976 June; 6(6): 16-18.

[DIGID 77A] With Help From Dual Microprocessors, Carousel Accesses 16 3M-type Cartridges. Digital Design. 1977 April; 7(4): 10-11.

[DIGID 77B] Bubbles debut in phone system announcer. Digital Design. 1977 May; 7(5): 22.

[DIGID 77C] Magnetic bubble memories. Digital Design. 1977 May; 7(5): 50-64.

[DIGID 77D] Minifloppy disk drive more than triples its capacity by using 77 tracks. Digital Design. 1977 May; 7(5): 82.

[DIGID 77E] Small double-sided double density disk. Digital Design. 1977 August; 7(9): 19.

[DIGID 77F] Technology Trends: Floppy Drive Innovations: Challenging Hard Disks. Digital Design. 1977 November; 7(11): 8, 10.

[DIGID 77G] Cartridge and Cassette Drives. Digital Design. 1977 December; 7(12): 58-59. 
[DIGID 77H] Rigid and Floppy Disk Drives. Digital Design. 1977 December; $7(12): 68,72$.

[DIGID 77I] Tape speed is up, but most systems need slow units. Digital Design. 1977 March; 7(3): 16.

[DORIL 76] Dorie, Larry A. Floppy disks the lowcost storage medium of the $70^{\prime} \mathrm{s}$. Data Management. 76 January; 14(1): 6-8.

[EARLJ 78] Early, James M. S. VLSI Technology. Spring Compcon 78 Sixteenth IEEE Computer Society International Conference: 1978 February 28-March 3: San Francisco, CA. New York: IEEE Computer Society; c 1978: (Section I) 1, 79, 102.

[EDN 77] Second generation minifloppy increases storage capacity by a factor of four. EDN. 1977 February 20; 22(4): 126.

[EDPI 74] EDP Industry Report. IBM 3850, NEE "OAK," Provides 472 Billion Bytes; Mass Storage Device Applies Virtual Storage Concept to 330 ; Newtonville, MA. International Data Corporation; 1974 October 17.

[EDPI 76] EDP Industry Report. IBM 3850 Shipments and Shakedowns Start to Roll Almost a Third of Large 370 Sites Expected to Install by 1978; Newtonville, MA. International Data Corporation; 1976 March 12.

[EETIM 78] Bubble Memories Are Applied in Air Force Avionics. Electronic Engineering Times. 1978 March 20; 125: 4.

[ELECN 74] See Floppy Disks Unprofitable Till '76. Electronic News. 1974 December 2: 43.

[ELECN 75] Floppy Disk Seen Firming in Second Half. Electronic News. 1975 May; 19: 51.

[ELECN 77A] Amperif Offers CCD Memory. Electronic News. 1977 January 10: 42 .

[ELECN 77B] Tape Drives Emerge From Shadow of Disk Growth. Electronic News. 1977 October; 3: 47.

[ELECT 76A] Data Handling: Floppy Disk in Miniature. Electronics. 1976 September 30; 49(20): 116.

[ELECT 76B] Prototype System Available From Rockwell. Electronics. 1976 September 30; 49(20): 29.

[ELECT 77A] Bubble Memory Gets NASA Check for Use in Space. Electronics. 1977 January 6; 50(1): 31-32. 
[ELECT 77B] Microprocessoos, Memories Dominate Digital Sessions. Electronics. 1977 Februaṛy 17; 50(4): 82-85.

[ELECT 77C] TI Raises Curtain on Its 65-K RAM. Electronics. 1977 April 14; 50(8): 49-50.

[ELECT 77D] Five Technologies Squeezing more Performance From LSI Chips. Electronics. 1977 August 18; 50(17): 92, 93.

[ELECT 77E] Two Heads Give Minifloppy On-Line 0.4 Megabyte Store. Electronics. 1977 November 24; 50(24): 41-42.

[EMM ND] Electronic Memories and Magnetics Corporation. Technical Manual Micromemory 3000 Memory System. Hawthorne, CA:

Electronic Memories and Magnetics; [n.d.]. 81p.

[EXCEL 76] Ex-Cell-0 Corporation (REMEX), Flexible Disk Drives: Data Sheet RFD 1000 Specification and Interface. Irvine, CA; 1976. [6 p.]; ED2-560/604.

[FARMV 75] Farmer, Vic. CDC 38500 Mass Storage System For Big 370s Makes NCC Debut. Computerworld. 1975 May 28; 9(22): 1-2.

[FETHG 76] Feth, George. Technology Trends: Moving-Head Disks at Performance Plateau. Digital Design. 1976 June; 6(6): $16,18$.

[FLORI 75] Flores, Ivan. The Drum. Peripheral Devices. Englewood Cliffs: Prentice Hall, Inc.; 1975: 437-447.

[FRANP 75] Franson, Paul. Floppy Disks Spin Into Systems. Electronics. 1975 January 23; 48(2): 59.

[FRASJ 75] Fraser, Jack. Disk and Tape-Mass Storage Moves in. Electronic News. 1975 June 23; 20(1033): 1, 38-39.

[FRASJ 76] Fraser, Jack; Rosenberg, Ron. Interest Rising in Small Diskette Market; Product Studies Under Way. Electronic News. 1976 September 13; 21(1097): 40 .

[FRASJ 77] Fraser, Jack. Tape Drives Business Enjoying Quiet Revival. Electronic News. 1977 October 3; 22(1152) 41, 46-47.

[FREEJ 77] Free, John. Here at last--disc players.' Popular Science. 1977 February; 210(2): 85-87, 140.

[GALUG 76] Galusha, Gary. Floppy disks: an update. Data Management. 76 January; 14(1): 10-12.

[GELLS 74] Geller, Sidney B. Archival Data Storage. Datamation. 1974 October; $20(10)$ : $72,75-76,80$. 
[GELLS 76] Geller, Sidney B. Erasing Myths About Magnetic Media. Datamation, 1976 March; 22(3): 65-68.

[GENEL ND] General Electric Company. BEAMOS (Beam-Addressed Metal Oxide Semiconductor). Schenectady, NY: General Electric Company; [n.d.]. $12 \mathrm{p}$.

[GILDJ 75] Gilder, Jules H. Mass Memories raising speed, dropping cost and storing billions of bytes. Electronic Design. 1975 October $25 ; 23(22): 46-50$.

[GROSR 76] Grossman, Robert M. "Micro" peripherals - moving to meet the microcomputer challenge. EDN. 1976 February 5; 21(3): $38,42-47$.

[GUIDM 78] Guidry, Mark R. Charge Coupled Device Digital Memory - A Forward Look. Spring Compcon 78 Sixteenth IEEE Computer Society International Conference; 1978 February 28-March 2; San Francisco, CA. New York: IEEE Computer Society; C1978: 144-155, 169.

[HAUFK 75] Haughton, Kenneth E. An Overview of Disk Storage Systems. Proceedings of IEEE. 1975 August; 63(8): 1148-1152.

[HELSA 70] Heller, Saul. Digital Computers Made Simple. Williston Park: Ameco Publishing; 1970. 128 p.

[HERBJ 76] Herbert, John P. Memory Prices Seen Dropping 40\%/Year Until '80. Computerworld. 1976 June 7: 57-58.

[HILLP 77] Hille, Peter F.; Holder, Carl. Avoiding Defective Diskettes. Mini-Micro System. 1977 February; 10(2): 54-55.

[HNATE 77] Hnatek, Eugene R. A. User's Handbook of Semiconductor Memories. New York, London, Sydney, Toronto: John Wiley and Sons (Wiley-Interscience Publication); 1977; 362, 372 p.

[HOAGO 72] Hoagland, Alberts. Mass Storage - Past, Present and Future. AFIPS Conference Proceedings; 1972 December 57; Anaheim, CA. AFIPS Press; c 1972: 985-991.

[HOBBL 76] Hobbs, L. C. Low-Cost Tape Devices. Computer. 1976 March; 9(3): 21-29.

[HOWIH 76] Howie, H. Robert, Jr. More Practical Applications of Trillion-Bit Mass Storage Systems. Spring Compcon 76: Twelfth IEEE Computer Society International Conference; 1976 February 24-26; San Francisco, CA. c 1976: 53-56.

[HUGHW 75] Hughes, William C., et. al. A Semiconductor Non-volatile Electron Beam Accessed Mass Memory. Proceedings of IEEE. 1975 August; 63(8): 1230-1240. 
[HUH 78] Hu, H. L. Bubbles--A New Magnetic Solid-State Technology. Spring Compcon 78 Sixteenth IEEE Computer Society International Conference; 1978 February 28-March 3; San Francisco, CA. New York: IEEE Computer Society; c 1978: 165, 166.

[HUNTF 54] Hunt, Frederick V. Electroacoustics: The Analysis of Transduction and Its Historical Background. Cambridge: Howard University Press (New York: John Wiley and Sons, Inc.); 1954: 37-40, 66-73.

[IDC 77] International Data Corporation Planning Service. OEM Storage Product Market. Waltham, MA: International Data Corporation (IDC); 1977 February. 69 p.

[KACZE 77] Kaczorowski, Edward M. Optical Mass Storage. Spring Compcon 77 Fourteenth IEEE Computer Society International Conference; 1977 February 28-March 3; San Francisco, CA. 33-36.

[KALSD 76] Kalstrom, David J. Simple Encoding Schemes Double Capacity of a Flexible Disc. Computer Design. 1976 September; 15(9): 98-102.

[KASHK 77] Kashkooli, Fred. MOS New Product Presentation. 1977 January 17 .

[KATZB 77] Katziue, Bob. Matching Magnetic Media With Modern Machines. Digital Design. 1977 June; 7(5): 20-34.

[KATZH 74] Katzan, Harry. Information Technology; the Human Use of Computers. New York: Petrocelli Books; 1974. 350 p.

[KELLJ 75] Kelly, John. The Development of an Experimental Electron-Beam-Addressed Memory Module. Computer. 1975 February; 8(2): 32-42.

[KENNG 77] Kenney, G.; Lou, D.; Wagner, J.: Zernike, F.; McFarlane, R.; Chan, A., An Experimental Optical Disc Data Recorder. Briarcliff Manor, NY: Philips Laboratories: A Division of North American Philips Corporation [1977]; N00014-76-C-0441. 17 p.

[KENVR 78] Kenville, Richard F. Optical Video Disc for Digital Mass Memory Applications. Spring Compcon 78 Sixteenth IEEE Computer Society International Conference; 1978 February 28-March 2; San Francisco, California. New York: IEEE Computer Society; c 1978: 170-172.

[KINGG 77] King, George. Cassette, Cartridge and Diskette Drives. Digital Design. 1977 June; 7(5): 50-90.

[KINNP 77] Kinnucan, Paul. A New Use for Core Memory. Mini-Micro Systems. 1977 March; 10(3): 23,26. 
[KRIER 76] Kriegler, Rudolph J. Recent Advances in MOS Technology. Telesis 1976/2. 1976 June; 4(6): 176-180.

[KRUSG 75] Kruschke, Gene. Memories Core and Semicon: Prices to Fall, Capacities to Rise. Digital Design. 1975 June; 5(6): 31.

[KURTA 77] Kurtzig, Arnie. Thin Film Heads for High Performance Disk Drives. Compcon Fall 77 Fifteenth IEEE Computer Society International Conference; 1977 September 6-9; Washington, D.C. Long Beach: IEEE Computer Society; c 1977: 124-127.

[LEAVD 77] Leavitt, Don; Bubble Device Put to the Test. Computerworld. 1977 February 7; $11(6): 1,5$.

[LOOME 75] Loomes, E. A. A Review of Magnetic Storage Devices. The Post Office Electrical Engineers Journal. 1975 July; 68(2): 96-102.

[MANLA 77A] Manildi, A. Bruce. Cassettes, A Mature Mass Memory for Microprocessors. Compcon 77 Fourteenth IEEE Computer Society International Conference 1977 February 28-March 3; San Francisco, CA. Long Beach: IEEE Computer Society; c 1977: 88-90.

[MANLA 77B] Manildi, A. Bruce. Designer's Guide for Selecting Magnetic Mini-Media. Computer Design. 1977 September; 16(9): 120a-120f.

[MANLA 77C] Manildi, A. Bruce. Inexpensive Digital Storage Media: Cassettes, Discs and Cartridges all have advantages. Let the application decide which one does your job. Electronic Design. 1976 December 6; 24(25): 86-88.

[MARCR 65] Marcucci, Richard. Design and Use of Recording Styli. Journal of Audio Engineering Society. 1965 April; 13(2): 130-133.

[MARTR 74] Martin, R. R.; Frankel, H. D. Forecast of Computer Memory Technology. Spring Compcon 74 Eighth IEEE Computer Society International Conference; 1974 February 26-28; San Francisco, CA. Northridge, IEEE Computer Society (New York, IEEE, Order Department); c 1974: 287-290.

[MARTR 75] Martin, R. R.; Frankel, H. D. Electronic Disks in the 1980's. Computer. 1975 February; 8(2): 24-30.

[MASSD 77] Massaro, Donald J. Advent of the Minifloppy (TM). Spring Compcon 77 Fourteenth IEEE Computer Society International Conference; 1977 February 28-March 3; San Francisco, CA. Long Beach: IEEE Computer Society; c 1977: 91-95. 
[MAVIC 77] Mavity, C. William. Bubble Memory System Applications . Anaheim, CA: Rockwell International Autonetics Group; 1977

February 28; International Electronics Convention Presentation.

5 p.

[MDATA 75A] A Cassette Competitor is Born. Modern Data. 1975 August; $8(8): 43$.

[MDATA 75B] Semiconductor Memories as Seen from Wall Street. Modern Data. 1975 September; 8(9): 46-47.

[MDATA 75C] Peripherals Make the Mini. Modern Data. 1975 December; $8(12): 34,43$.

[MDATA 76A] Laser Mass Memory for Sensitive Agencies. Modern Data. 1976 February; 9(2): 10.

[MDATA 76B] The HP 9825A. Modern Data. 1976 March. 9(3): 52.

[MICRB ND] Micro-Bit Corporation. Micro-Bit 700 Electron Beam Accessed Memories. Lexington, MA; [n.d.]. 8 p.

[MIDDJ ND] Middlestaedt, Jack; Fabri-Tek Inc. Model 760 Memory Requirements Survey. Minneapolis, MN; [n.d.]. 4 p.

[MIDDS 76] Middelhoek, S.; George, P. K.; Dekker, P. Physics of Computer Memory Devices. London, New York, San Francisco: Academic Press; 1976. 1-137, 402, 647 p.

[MINCD 76A] Mincom Division 3M Company. DCD-1 Data Cartridge Drive (Price List: effective July 12, 1976). St. Paul, MN; 1976. 1 p.; RM-DCD-1-PL (76.05)R .

[MINCD 76B] Mincom Division 3M Company. DCS-3000 Data Cartridge System (Price List: effective December 1, ]976). St. Paul, MN; 1976. 1 p.; RM-DCS-3-PL-1 (116.075) R2.

[MINCD NDB] Mincom Division 3M Company. 3M Got There First Again. St. Paul, MN; [n.d.]. 1 p.

[MINCD NDA] Mincom Division 3M Company. In 1971, 3M Accomplished the Difficult. The Impossible Took a Little Longer. St. Paul, MN; [n.d.] 3 p.

[MINIC 75] Which Will Prevail: Cassettes or Cartridges? Answer: The Floppy Disk, Says Data Report. Minicomputer News. 1975 April; 24: 18.

[MINIC 76B] Minifloppy Offers Random Access in a Cassette Drive Sized Package. Minicomputer News. 1976 September; 23: 1. 
[MINIM 76A] Memory Movements. Mini-Micro System. 1976 August; 9(8): 42 .

[MINIC 76A] Tape Drive Technology Still Quietly Advancing. Minicomputer News. 1976 July 29; 2(16): 5.

[MINIM 76B] A New Disk Head Develops. Mini-Micro Systems. 1976 August; $9(8): 43$.

[MINIM 76C] A Mini Floppy for Micros. Mini-Micro Systems. 1976 October; 9(]0): 26-27.

[MINIM 78A] The Outlook for Bubble Memory Devices, Mini-Micro Systems. 1978 February; $11(2): 32,33$.

[MITCS 76] Mitchell, Stuart G. Defense Systems Management School: Microprocessor Technology for Managers. Fort Bevoir, VA: National Technical Information Service; 1976 May; PMC 76-1. $38 \mathrm{p}$.

[MORGW 75] Morgan, William. Requirements of a Mass Storage System. Data Processing. 1975 November/December; 17(5): 304-308.

[OLDHW 77] Oldham, William G. The Fabrication of Micro-electronic Circuits. Scientific American. 1977 September; 237(3): 111-128.

[OLSOH 52] 0lson, Harry F. Elements of Acoustical Engineering. Toronto, New York, London: D. Van Nostrand Company, Inc.; 1952 May. 304-310.

[OLSOK 75] Olson, Kirtland. Chipping Away at Core: Semiconductor and Core Memories. Digital Design. 1975 January; 5(1): 13-20.

[PARKH] Parks, H. G.; Hughes, W. C.; Possin, G. E.; Lemmond, C. Q.; Kirkpatrick, C. G.; Ellis, G. W.; Wilson, R. H. BEAMOS--An Electron Beam Digital Memory Device Using Matrix Lens Optics. Schenectady, NY; General Electric Company Corporate Research and Development; [n.d.]; [n.a.]. Schenectady, NY; [n.a.].

[PEARC 67] Pear, C. B., Jr. Magnetic Recording in Science and Industry. New York, Amsterdam, London: Reinhold Publishing Corp.; 1967. 453 p.

[PELLS 76A] Pellerin, Sharon. Floppy disk Revolution Just Getting Started. Minicomputer News. 1976 July 29; 2(16): 6.

[PELLS 76B] Pellerin, Sharon. On-Line Storage Needs Growing at Rapid Pace. Minicomputer News. 1976 July 29; 2(16): 2.

[PERLA 77] Perlman, Al. Await Double-Density Floppy Standard. Electronic News. 1977 October 3; 22(1152): 40,42,44. 
[PERPH 76] Intel Introduces Single Board Display Memory System. Peripherals Weekly. 1976 January 14; 9(3): 7.

[PERPH 77] Floppy Disk Market Growth Immune to Solid State Advances Says VDC Study. Peripheral Weekly. 1977 July 20; 10(30): 1-3.

[PI 77] Precision Instrument Company. System 1800: Functional Description. Santa Clara, CA: Precision Instrument Company; 1977 August 15. $25 \mathrm{p}$.

[POES 76] Poe, Stephen. Photographic Video Disc Technology Assessment. Washington, D.C.: Government Printing Office; 1976 October; N00600-76-C-0505. $518 \mathrm{p}$.

[POSSG ND] Possin, G. E. et al. BEAMOS - A New Electron Beam Digital Memory Device. Schenectady, NY; Corporate Research and

Development, General Electric Company; [n.d.]; DAAB07-72-C-0098. 4 p. $(305-308)$.

[POWED 77] Powell, Dave. Storage Devices: Disks Faster But Floppy Speed to Grow With Density. Minicomputer News. 1977 July; 28: $1,3,17$.

[PUSHP NDA] Pushpa International Corporation. Logical Address Space Enhancement Option. Westminster, CA: [n.d.]. 6 p.

[PUSHP NDB] Pushpa International Corporation. State-of-the-Art Single Card Core Memory Systems. Westminster, CA; [n.d.]. 4 p.

[PUTHS 78] Puthuff, S. H. The Dynamic State of Storage Technology. Seminar Presentation at National Bureau of Standards; 1978 February 1; Gaithersburg, MD. Santa Clara: Memorex Corporation; c 1977: l-20 p.

[QANTE ND] Qantex Division North Atlantic Industries, Inc. The Minidrive for the Minicartridge. [n.d.]. $1 \mathrm{p}$.

[RAMPI 77] Rampil, Ira. A Floppy Disk Tutorial. BYTE. 1977 December; 2(12).

[RECIS 77A] Recicar, Steve A. (Interviewer). Interview with Disk Memory Manufacturer 1977 September 27.

[RECIS 77B] Recicar, Steve A. (Interviewer). Interview with Drum Memory Manufacturer 1977 September 27.

[RECIS 77C] Recicar, Steve A. (Interviewer). Phone Interview with Disk Memory Manufacturer, 1977 December 8.

[RECIS 77D] Recicar, Steve A. (Interviewer). Phone Interview with Rich Matlack of Dataquest. 1977 December 8. 
[RECIS 77E] Recicar, Steve A. (Interviewer). Phone Interview with Nancy Skull of International Data Corporation (ICD). 1977 December 13.

[RECIS 78A] Recicar, Steve. Phone Interview with Disk Memory Manufacturer. 1978 January 30.

[RECIS 78B] Recicar, Steve. Phone Interview with Disk Memory Manufacturer. 1978 February 16.

[RECIS 78C] Recicar, Steve A. (Interviewer). Phone Interview with Cartridge Disk Memory Manufacturer. 1978 March 28.

[REICD 75] Reichel, Donald R. Flexible Discs: A Look at the Latest Mini-Peripheral. EDN. 1975 January 20; 20(2): 37-40.

[RECHA 76] Richards, Alan J. Mini Data Cartridge: A Convincing Alternative for Low-Cost, Removable Storage. Hewlett-Packard Journal. 1976 May; 27(9): 6-7.

[RICHR 57] Richards, R. K. Digital Computer Components and Circuits. Princeton, Toronto, London, New York: D. Van Nostrand Inc.; 1957: 336-342.

[RICKP 77] Ricketts, Phillip. In Choosing Magnetic Media: Key Factors in Storage Choice? Access and Cost. 1977 September 26; 11(39): $\mathrm{S} / 2$.

[ROCKW ND] Rockwell International. POS/8 Bubble Memory System. Anaheim, CA; [n.d.]. 8 p.

[RODRJ 75] Rodriguez, Juan A. An Analysis of Tape Drive Technology. Proceedings of IEEE. 1975 August; 63(8): 1153-1159.

[ROSCT 77] Roscamp, Thomas A.; Frank, Paul D. Thin-film Magnetic Heads Excel in Packing and Moving Data. Electronics. 1977 March 3; $50(5): 97-103$.

[SCHNN 74] Schneidewind, Norman; Syms, Gordon. Mass Memory System Peripherals. Spring Compcon 74 Eighth IEEE Computer Society International Conference; 1974 February 26-28; San Francisco, CA. Long Beach, IEEE Computer Society; c 1974: 87-90.

[SCRUS 76] Scrupski, Stephen E. Small Systems move into the design spotlight. Electronics. 1976 October 28; 49(22): 90-96.

[SHAPR 77] Shapiro, Richard B. The Controller: Key to Floppy Disk Performance. Mini-Micro Systems. 1977 April; 10(4): 28-41. 
[SHELC 74] Shelton, Carl F.; Brown, Byron R. Advances in Optical Storage - An Alternate Technology. Compcon 74 Eighth IEEE Computer Society International Conference; 1974 February 26-28; San Francisco, CA. Long Beach, IEEE Computer Society; c 1974: 97-98.

[SHUGA 77A] Shugart Associates. SA800/801 Diskette Storage Drive OEM Manual. Sunnyvale, CA; 1977 January. [39 p.]; 50574-2.

[SHUGA 77B] Shugart Associates. SA400 Minifloppy Diskette Storage Drive. Sunnyvale, CA; 1977 February. [40 p.]; 54102-1.

[SHUGA ND] Shugart Associates. The Gap is Gone. The SA400 Minifloppy Disk Drive. Sunnyvale, CA; [n.d.]. [4 p.].

[SMITD 78] Smith, D. 0. Electron Beam Accessed Memory. Spring Compcon 78 Sixteenth IEEE Computer Society International Conference; 1978 February 28-March 3; San Francisco, CA, New York: IEEE Computer Society; c1978: 167-169.

[SOLOL 75] Solomon, Lewis; Ross, Edward; Switenbank, Thomas. The Cartridge-Cassette Confrontation. Digital Design. 1975 May; $5(5): 24-29$.

[SPELD 75] Speliotis, Dennis E. Bridging the Memory Access Gap. AFIPS Conference Proceedings: 1975 National Computer Conference; 1975 May 19-22; Anaheim, CA. Montvale, NJ; cl975: 501-508.

[SPRAH 64] Spratt, Hector G. M. Magnetic Tape Recording. Toronto, New York, London: D. Van Nostrand Company, Inc.; 1964: 123-151 p.

[STEWW 58] Stewart, W. Earl. Magnetic Recording Technique. New York, Toronto, London: McGraw-Hill Book Company, Inc.; 1958. 128 p.

[STODD 75] Stoddard, David L. Lower Cost in Near Future. Aim of Floppy Developers. Computerworld.' 1975 April 9: 35.

[STORA ND] Storage Technology Corporation. STC 8000 Series Disk Subsystems. Louisville, Co; [n.d.]. [6 p.].

[STRIA 77] Stricklin, Alan. A Videodisc for Education and Industry. Videography. 1977 February. 2(2): 28-29.

[SURDE 77] Surdan, Esther. Memory for DG Novas Ampex Unit Replaces Fixed-Head Disks. Computerworld. 1977 January 31; 11(5): 43.

[TASCA 76] Tasch, Al F.; Frye, C. Robert; Fu, Horng-Sen. The ChargeCoupled RAM cell Concept. IEEE Transaction Electron Devices. 1976 February; ED-23: 126-131. 
[TEXAS NDA] Texas Instruments Incorporated Semiconductor Group. Magnetic Bubble Memories and System Interface Circuits from Texas Instruments. Dallas, TX: Texas Instruments Incorporated; 1977 February. $62 \mathrm{p}$.

[TEXAS NDB] Texas Instruments. Product Announcement New "Silent 700" Model 763/765 Memory Terminal. Houston, TX; [n.d.]. [4 p.].

[THEID 78] Theis, Douglas J. An Overview of Memory Technologies. Datamation. 1978 January; 24(1): 113-131.

[THURR 76] Thuras, Rodger D. New Magnetic Materials Help Core Memories Stay Alive. Computer Design. 1976 July; 15(7): 100-102.

[TURNR 74] Turn, Rein. Computers in the 1980s. New York, London: Columbia University Press; 1974: 196-201.

[UPTOM 75A] Upton, Molly. Shugart Expects 1976 Sales to Climb to $\$ 9$ Million. Computerworld. 1975 November 19: 51.

[UPTOM 75B] Upton, Molly. Venture Predictions Realistic: Vendors Say Floppy Business Picking Up. Computerworld. 1975 August 27: 33-34.

[VADAL 71] Vadasz, L. L.; Chua, H. T.; Grove, A. S. Semiconductor Random-Accesss Memories. IEEE Spectrum. 1971 May; 8: 40-48.

[VAUGF 77] Vaughan, Frank. Burroughs Unveils "Supercomputer" with Array Design. Computerworld. 1977 April 11: 39.

[VITTJ 75] Vittera, Jim. Nonvolatile microprocessor Memory Uses $4 \mathrm{~K} \mathrm{n}$ Channel MOS RAMs. EDN. 1975 April 20; 20(8): 53-56.

[VORNL 71] Vornerin, Lawrence J. Approaches for Making Bubble Domain Materials. IEEE Transactions on Magnetics. 1971 September; 7 : 404-409.

[WAGC ND] Wangco, Inc. Wangco Micro-Floppy Model 82. Los Angeles, CA; [n.d.]. [2 p.].

[WARNR 77A] Warnar, Robert B. J.; Calomeris, Peter J. Foreign and Domestic Accomplishments in Magnetic Bubble Device Technology. Computer Science and Technology. 1977 January; Nat. Bur. Stand. (U.S.) Spec. Publ. 500-1, CODEN: NBSAV.

[WARNAR 77B] Warnar, Robert B. J. (Interviewer). Phone Interview with CCD Memory Manufacturer. 1977 March 2l. 7 p.

[WARNR 77C] Warnar, Robert B. J. (Interviewer). Phone Interview with CCD Memory Manufacturer. 1977 March 22. 7 p. 
[WARNR 77D] Warnar, Robert B. J. (Interview). Interview with MOS Manufacturer. 1977 March 23.

[WARNR 77E] Interviewer). Phone Interview with CCD Memory Manufacturer. 1977 March 23. 7 p.

[WARNR 77F] Warnar, Robert B. J. (Interviewer). Phone Interview with Magnetic Bubble Manufacturer. 1977 March 24. 7 p.

[WARNR 77G] Warnar, Robert B. J.; Calomeris, Peter J. (Interviewers). Interview with MOS Memory Manufacturer. 1977 March 25 and April 1. 7 p.

[WARNR 77H] Warnar, Robert B. J. (Interviewer). Interview with Core Memory Manufacturer. 1977 March 28. 7 p.

[WARNR 77I] Warnar, Robert B. J.; Calomeris, Peter J. Interview with Core Manufacturer. 1977 March 30. 7 p.

[WARNR 77J] Warnar, Robert B. J. Impact of Charge Transfer Device Technology on Computer System. Computer Science and Technology. 1977 April; Nat. Bur. Stand. (U.S.), Spec. Publ. 5005. Coden: XNBSAV.

[WARNR 77K] Warnar, Robert B. J. (Interviewer). Phone interview with Core Memory Manufacturer. 1977 May 14. p 7.

[WARNR 77L] Warnar, B. J. (Interviewer). Phone Interview with CCD Memory Manufacturer. 1977 May 23. 7 p.

[WARNR 77M] Warnar, Robert B. J. (Interviewer). Interview with Core Memory Manufacturer. 1977 May 24. 7 p.

[WARNR 77N] Warnar, Robert B. J. (Interviewer). Phone Interview with Core Memory Manufacturer. 1977 May 24. 7 p.

[WARNR 770] Warnar, Robert B. J. (Interviewer). Interview with Magnetic Bubble Memory Manufacturer. 1977 May 25. 7 p.

[WARNR 77P] Warnar, Robert B. J. (Interviewer). Phone Interview with Core Memory Manufacturer. 1977 May 26.・ 7 p.

[WARNR 77Q] Warnar, Robert B. J. (Interviewer). Phone interview with Magnetic Bubble Manufacturer. 1977 May 26. 7 p.

[WARNR 77R] Warnar, Robert B. J. (Interviewer). Phone Interview with Core Memory Manufacturer. 1977 May 27. 7 p.

[WARNR 77S] Warnar, Robert B. J.; Calomeris, Peter J. Technology Assessment of Computer Memories. Dimensions. 1977 August; 61(8): 24-26. 
[WARNR 77T] Warnar, Robert B. J. (Interviewer). Phone Interview with EBAM Memory Manufacturer. 1977 August 18.

[WARNR 77U] Warnar, Robert B. J. (Interviewer). Interview with Core Memory Manufacturer. 1977 September 21. 7 p.

[WARNR 77V] Warnar, Robert B. J. (Interviewer). Interview with Bulk Core Memory Manufacturer. 1977 September 7.7 p.

[WEITC 70] Weitzman, Cay. Optical Technologies for Future Computer System. Computer Design. 1970 April: 169-175.

[WILDM 75] Wildmann, Manfred. Terabit Memory Systems: A Design History. Proceedings of the IEEE. 1975 August; 63(8): 1160-1165.

[WORLE 75] Worley, Ernest. Core Memory State-of-the-Art. Modern Data. 1975 August; 8(8): 54.

[YPMAJ 75] Ypma, J. E.; Gergis, I. S.; Archer, J. L. 64K Fast Access Chip Design. Anaheim, CA; Rockwell International; 1975 December 1; Annual Conference on Magnetism and Magnetic Materials Presentation. $8 \mathrm{p}$. 


U.S. DEPT. OF COMM.
BIBLIOGRAPHIC DATA
SHEET

4. TITLE AND SUBTITLE

COMPUTER SCIENCES \& TECHNOLOGY:

COMPUTER PERIPHERAL MEMORY SYSTEM FORECAST

\section{AUTHOR(S)}

Robert B. J. Warnar, Peter J. Calomeris, and Steve A. Recicar
1. PUBLICATION OR REPORT NO.

NBS SP $500-45$

5. Publication Date

Ap,ril 1979

6. Performing Organization Code

8. Performing Organ. Report No.

$$
650
$$

10. Project/Task/Work Unit No.

NATIONAL BUREAU OF STANDARDS

DEPARTMENT OF COMMERCE

WASHINGTON, DC 20234

11. Contract/Grant No.

12. SPONSORING ORGANIZATION NAME AND COMPLETE ADDRESS (Street, City, State, ZIP)

Same as Item 9

13. Type of Report \& Period Covered

Final through 1978

14. Sponsoring Agency Code

\section{SUPPLEMENTARY NOTES}

This forecast covers thirteen peripheral memory technologies.

Library of Congress Catalog Card Number: 79-600036.

$\square$ Document describes a computer program; SF-185, FIPS Software Summary, is attached.

16. ABSTRACT (A 200-word or less factual summary of most significant information. If document includes a significant bibliography or literature survey, mention it here.)

This document describes and forecasts computer peripheral memory

technologies as displayed by U.S. research and manufacturing

facilities. Specifically, both technical and economic criteria are

discussed. The presented peripheral memories include contemporary

and emerging systems, all of which are compared in graphs, tables,

and decision trees. The document contains an extensive bibliography

(in ANSI format) to support certain main points that are supplemented

by information supplied by the Institute for Computer Sciences and

Technology (ICST) resources. Additional information and verification

was received from private interviews with various U.S. technical

experts and equipment manufacturers.

17. KEY WORDS (six to twelve entries; alphabetical order; capitalize only the first letter of the first key word unless a proper name; seperated by semicolons)

Beam-addressables; cartridges; cassettes; core memories; disks; drums; floppy disks; laser beams; magnetic bubbles; masstapes; solid state memories; video disks.

18. AVAILABILITY CXnlimited

For Official Distribution. Do Not Release to NTIS

X Order From Sup. of Doc., U.S. Government Printing Office, Washington, DC 20402, SD Stock No. SNÓ03-00302039-7

Order From National Technical Information Service (NTIS), Springfield, VA. 22161

\begin{tabular}{|c|c|}
\hline $\begin{array}{c}\text { 19. SECURITY CLASS } \\
\text { (THIS REPORT) } \\
\text { X } \\
\text { UNCLASSIFIED }\end{array}$ & 147 \\
\hline $\begin{array}{c}\text { 20. SECURITY NOLASS OF } \\
\text { (THIS PAGE) } \\
\text { PRINTED PAGES } \\
\text { UNCLASSIFIED }\end{array}$ & 22. Price \\
$\$ 3.25$ \\
\hline
\end{tabular}




\section{ANNOUNCEMENT OF NEW PUBLICATIONS ON COMPUTER SCIENCE \& TECHNOLOGY}

Superintendent of Documents, Government Printing Office, Washington, D. C. 20402

\section{Dear Sir:}

Please add my name to the announcement list of new publications to be issued in the series: National Bureau of Standards Special Publication 500-.

Name

Company

Address

City State Zip Code

(Notification key N-503) 


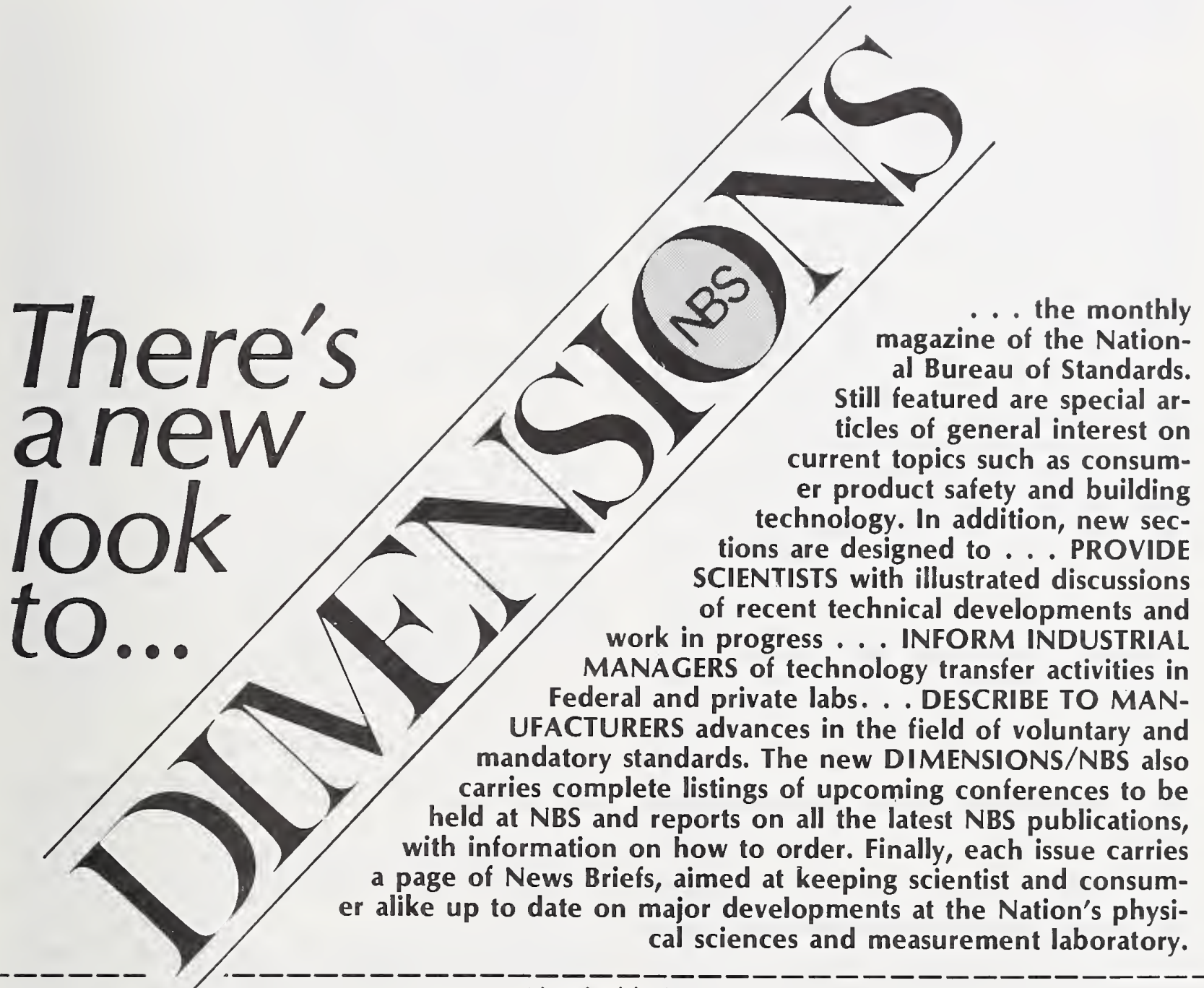

(please detach here)

SUBSCRIPTION ORDER FORM

Enter my Subscription To DIMENSIONS/NBS at \$12.50. Add \$3.15 for foreign mailing. No additional postage is required for mailing within the United States or its possessions. Domestic remittances should be made either by postal money order, express money order, or check. Foreign remittances should be made either by international money order, draft on an American bank, or by UNESCO coupons.

Send Subscription to:

\section{[}

NAME-FIRST, LAST

\section{긴}

COMPANY NAME OR ADDITIONAL ADDRESS LINE
Remittance Enclosed (Make checks payable to Superintendent of Documents)

Charge to my Deposit Account No.
MAIL ORDER FORM TO: Superintendent of Documents Government Printing Office Washington, D.C. 20402 



\section{NBS TECHNICAL PUBLICATIONS}

\section{PERIODICALS}

JOURNAL OF RESEARCH-The Journal of Research of the National Bureau of Standards reports NBS research and development in those disciplines of the physical and engineering sciences in which the Bureau is active. These include physics, chemistry, engineering, mathematics, and computer sciences. Papers cover a broad range of subjects, with major emphasis on measurement methodology, and the basic technology underlying standardization. Also included from time to time are survey articles on topics closely related to the Bureau's technical and scientific programs. As a special service to subscribers each issue contains complete citations to all recent NBS publications in NBS and nonNBS media. Issued six times a year. Annual subscription: domestic $\$ 17.00$; foreign $\$ 21.25$. Single copy, $\$ 3.00$ domestic; $\$ 3.75$ foreign.

Note: The Journal was formerly published in two sections: Section A "Physics and Chemistry" and Section B "Mathematical Sciences."

\section{DIMENSIONS/NBS}

This monthly magazine is published to inform scientists, engineers, businessmen, industry, teachers, students, and consumers of the latest advances in science and technology, with primary emphasis on the work at NBS. The magazine highlights and reviews such issues as energy research, fire protection, building technology, metric conversion, pollution abatement, health and safety, and consumer product performance. In addition, it reports the results of Bureau programs in measurement standards and techniques, properties of matter and materials, engineering standards and services, instrumentation, and automatic data processing.

Annual subscription: Domestic, \$11.00; Foreign \$13.75

\section{NONPERIODICALS}

Monographs-Major contributions to the technical literature on various subjects related to the Bureau's scientific and technical activities.

Handbooks-Recommended codes of engineering and industrial practice (including safety codes) developed in cooperation with interested industries, professional organizations, and regulatory bodies.

Special Publications-Include proceedings of conferences sponsored by NBS, NBS annual reports, and other special publications appropriate to this grouping such as wall charts, pocket cards, and bibliographies.

Applied Mathematics Series-Mathematical tables, manuals, and studies of special interest to physicists, engineers, chemists, biologists, mathematicians, computer programmers, and others engaged in scientific and technical work.

National Standard Reference Data Series-Provides quantitative data on the physical and chemical properties of materials, compiled from the world's literature and critically evaluated. Developed under a world-wide program coordinated by NBS. Program under authority of National Standard Data Act (Public Law 90-396).
NOTE: At present the principal publication outlet for these data is the Journal of Physical and Chemical Reference Data (JPCRD) published quarterly for NBS by the American Chemical Society (ACS) and the American Institute of Physics (AIP). Subscriptions, reprints, and supplements available from ACS, 1155 Sixteenth St. N.W., Wash., D.C. 20056.

Building Science Series-Disseminates technical information developed at the Bureau on building materials, components, systems, and whole structures. The series presents research results, test methods, and performance criteria related to the structural and environmental functions and the durability and safety characteristics of building elements and systems. Technical Notes-Studies or reports which are complete in themselves but restrictive in their treatment of a subject. Analogous to monographs but not so comprehensive in scope or definitive in treatment of the subject area. Often serve as a vehicle for final reports of work performed at NBS under the sponsorship of other government agencies. Voluntary Product Standards-Developed under procedures published by the Department of Commerce in Part 10, Title 15, of the Code of Federal Regulations. The purpose of the standards is to establish nationally recognized requirements for products, and to provide all concerned interests with a basis for common understanding of the characteristics of the products. NBS administers this program as a supplement to the activities of the private sector standardizing organizations.

Consumer Information Series-Practical information, based on NBS research and experience, covering areas of interest to the consumer. Easily understandable language and illustrations provide useful background knowledge for shopping in today's technological marketplace.

Order above NBS publications from: Superintendent of Documents, Government Printing Office, Washington, D.C. 20402.

Order following NBS publications-NBSIR's and FIPS from the National Technical Information Services, Springfield, Va. 22161.

Federal Information Processing Standards Publications (FIPS PUB)-_Publications in this series collectively constitute the Federal Information Processing Standards Register. Register serves as the official source of information in the Federal Government regarding standards issued by NBS pursuant to the Federal Property and Administrative Services Act of 1949 as amended, Public Law 89-306 (79 Stat. 1127), and as implemented by Executive Order 11717 (38 FR 12315, dated May 11, 1973) and Part 6 of Title 15 CFR (Code of Federal Regulations).

NBS Interagency Reports (NBSIR)-A special series of interim or final reports on work performed by NBS for outside sponsors (both, government and non-government). In general, initial distribution is handled by the sponsor; public distribution is by the National Technical Information Services (Springfield, Va. 22161) in paper copy or microfiche form.

\section{BIBLIOGRAPHIC SUBSCRIPTION SERVICES}

The following current-awareness and literature-survey bibliographies are issued periodically by the Bureau:

Cryogenic Data Center Current Awareness Service. A literature survey issued biweekly. Annual subscription: Domestic, \$25.00; Foreign, \$30.00.

Liquified Natural Gas. A literature survey issued quarterly. Annual subscription: $\$ 20.00$.
Superconducting Devices and Materials. A literature survey issued quarterly. Annual subscription: $\$ 30.00$. Send subscription orders and remittances for the preceding bibliographic services to National Bureau of Standards, Cryogenic Data

Center (275.02) Boulder, Colorado 80302. 
U.S. DEPARTMENT OF COMMERCE

National Bureau of Standards

Washington, D.C. 20234

OFFICIAL BUSINESS

Penalty for Private Use, $\$ 300$
POSTAGE AND FEES PAID US. DEPARTMENT OF COMMERCE COM-215

SPECIAL FOURTH-CLASS RATE SOOK 